\title{
A derived approach to geometric McKay correspondence in dimension three
}

\author{
By Sabin Cautis at Houston and Timothy Logvinenko at Stockholm
}

\begin{abstract}
We propose a three dimensional generalization of the geometric McKay correspondence described by Gonzales-Sprinberg and Verdier in dimension two. We work it out in detail in abelian case. More precisely, we show that the Bridgeland-King-Reid derived category equivalence induces a natural geometric correspondence between irreducible representations of $G$ and subschemes of the exceptional set of $G$-Hilb $\left(\mathbb{C}^{3}\right)$. This correspondence appears to be related to Reid's recipe.
\end{abstract}

\section{Introduction}

The study of the McKay correspondence began with an observation by John McKay in [11] that there exists a bijective correspondence between irreducible representations of a finite subgroup $G \subseteq \mathrm{SL}_{2}(\mathbb{C})$ and exceptional divisors of the minimal resolution $Y$ of $\mathbb{C}^{2} / G$. Gonzales-Sprinberg and Verdier in [6] gave a geometric construction of this correspondence. The aim of this paper is to give a generalization of this construction for dimension three. Our approach is via the derived McKay equivalence of [1] and for $G$ abelian it appears to give a categorification of 'Reid's recipe' from [13].

The original construction of [6] is as follows. Denote by $K^{G}\left(\mathbb{C}^{2}\right)$ the Grothendieck ring of $G$-equivariant coherent sheaves on $\mathbb{C}^{2}$ and by $K(Y)$ the Grothendieck ring of $Y$. Let $\mathscr{M} \in \operatorname{Coh}\left(Y \times \mathbb{C}^{2}\right)$ be the structure sheaf of the reduced fiber product $Y \times{ }_{\mathbb{C}^{2} / G} \mathbb{C}^{2}$. Define the transform $\Theta: K^{G}\left(\mathbb{C}^{2}\right) \rightarrow K(Y)$ by

$$
\Theta(-)=\left(\pi_{Y *}\left(\mathscr{M} \otimes \pi_{\mathbb{C}^{2}}^{*}(-)\right)\right)^{G}
$$

where $\pi_{Y}$ and $\pi_{\mathbb{C}^{2}}$ are the projections from $Y \times \mathbb{C}^{2}$ to $Y$ and $\mathbb{C}^{2}$.

Denote by $\operatorname{Exc}(Y)$ the set of irreducible exceptional divisors on $Y$. Let $\rho$ be an irreducible representation of $G$. Since $\mathscr{M}$ is flat over $Y$ its pushforward to $Y$ is a vector bundle and $\mathscr{L}_{\rho}:=\Theta\left(\mathcal{O}_{\mathbb{C}^{2}} \otimes \rho^{\vee}\right)$ is its $\rho$-eigensheaf. In general $\mathscr{L}_{\rho}$ is a vector bundle of dimension $\operatorname{dim}(\rho)$ and is called a tautological or GSp- $V$ sheaf. It is proven in [6] that: 
(1) $K^{G}\left(\mathbb{C}^{2}\right) \stackrel{\Theta}{\rightarrow} K(Y)$ is an isomorphism of abelian groups.

(2) $\left\{c_{1}\left(\mathscr{L}_{\rho}\right) \mid \rho \in \operatorname{Irr}(G)\right.$ non-trivial $\}$ is the basis of $H^{2}(Y, \mathbb{Z})$ dual to the basis $\{E \mid \operatorname{Exc}(Y)\}$ of $H_{2}(Y, \mathbb{Z})$. This gives a 1-to-1 correspondence between non-trivial $\rho$ and elements of $\operatorname{Exc}(Y)$. Denote by $E_{\rho}$ the irreducible exceptional divisor dual to $c_{1}\left(\mathscr{L}_{\rho^{v}}\right)$.

(3) For each non-trivial $\rho \in \operatorname{Irr}(G)$ we have

$$
\Theta\left(\mathcal{O}_{0} \otimes \rho\right)=\left[\mathcal{O}_{E_{\rho}}\right]
$$

where $\mathcal{O}_{0}$ is the skyscraper sheaf at the origin $(0,0) \in \mathbb{C}^{2}$.

Recall that a resolution $X^{\prime} \stackrel{f}{\rightarrow} X$ is called crepant if $f^{*} \omega_{X}=\omega_{X^{\prime}}$ (see [12] for more details). In dimension two the minimal resolution $Y \rightarrow \mathbb{C}^{2} / G$ is the unique crepant resolution. In dimension three and above crepant resolutions may not exist and are not necessarily unique. In particular $\mathbb{C}^{3} / G$ has a number of crepant resolutions connected by a chain of flops.

To figure out which crepant resolution to consider it helps to go back to the minimal resolution $Y \rightarrow \mathbb{C}^{2} / G$ and observe that $Y \cong G$-Hilb $\left(\mathbb{C}^{2}\right)$, the fine module space of $G$ clusters in $\mathbb{C}^{2}$. A $G$-cluster in $\mathbb{C}^{n}$ is a $G$-invariant subscheme $\mathbb{Z} \subseteq \mathbb{C}^{n}$ such that $H^{0}\left(\mathcal{O}_{Z}\right)$ is isomorphic to the regular representation $V_{\text {reg }}$ of $G$. The structure sheaf $\mathscr{M}$ of the reduced fiber product $Y \times_{\mathbb{C}^{2} / G} \mathbb{C}^{2}$ turns out to be the universal family of $G$-clusters over $Y \times \mathbb{C}^{2}$.

This suggests that for $G \subseteq \mathrm{SL}_{3}(\mathbb{C})$ we should take $Y$ to be $G-\mathrm{Hilb}\left(\mathbb{C}^{3}\right)$ and $\mathscr{M}$ to be the universal family of $G$-clusters over $Y \times \mathbb{C}^{3}$. Given a variety $X$ denote by $D(X)$ (resp. $\left.D^{G}(X)\right)$ the derived category of coherent sheaves on $X$ (resp. $G$-equivariant coherent sheaves on $X$ if $X$ is equipped with an action of $G)$. Define $\Phi: D(Y) \rightarrow D^{G}\left(\mathbb{C}^{3}\right)$ to be the integral transform

$$
\Phi(-)=\pi_{\mathbb{C}^{3} *}\left(\mathscr{M} \stackrel{\mathbf{L}}{\otimes} \pi_{Y}^{*}\left(-\otimes \rho_{0}\right)\right)
$$

with kernel $\mathscr{M}$, where $\left(-\otimes \rho_{0}\right)$ is the functor of equipping the sheaf with the trivial $G$ action. It was proven in [1] that $\Phi$ is an equivalence of derived categories and consequently that $Y$ is a crepant resolution of $\mathbb{C}^{3} / G$. As derived equivalence implies $K$-group isomorphism this gives a very satisfying proof of (1) in dimension three.

There has been some effort to generalise (2). One can define the vector bundles $\mathscr{L}_{\rho}$ for $\rho \in \operatorname{Irr}(G)$ as before. Since $\left\{\mathcal{O}_{\mathbb{C}^{3}} \otimes \rho\right\}$ forms a basis for $K^{G}\left(\mathbb{C}^{3}\right)$ the collection $\left\{\mathscr{L}_{\rho}\right\}$ is a basis for $K(Y)$. Hence the Chern classes $c_{1}\left(\mathscr{L}_{\rho}\right)$ span $H^{2}(X, \mathbb{Z})$. But they do not form a basis since there are relations.

When $G$ is abelian the bundles $\mathscr{L}_{\rho}$ are line bundles and $Y$ is a toric variety. Following a conjecture by Reid, Craw showed in [4] that certain $\mathscr{L}_{\rho}$ whose classes are redundant can be replaced by abstract elements of $K(Y)$ in such a way that second Chern classes of these 
'virtual' bundles give a basis of $H^{4}(Y, \mathbb{Z})$. This gives a 1-to-1 correspondence between $\operatorname{Irr}(G)$ and a basis of $H^{*}(Y, \mathbb{Z})$.

What dictates the way 'virtual' bundles are produced is the so-called 'Reid's recipe' (see [13], Section 6, and [4], Section 3). It is a somewhat ad-hoc way, based on calculation of monomial bases of each of the toric affine charts of $Y$, to assign a character of $G$ to each exceptional curve of the form $E \cap F$ for $E, F \in \operatorname{Exc}(Y)$ and a character or a pair of characters to each exceptional divisor $E \in \operatorname{Exc}(Y)$.

We propose generalizing (3). Take the inverse $\Psi: D^{G}\left(\mathbb{C}^{3}\right) \rightarrow D(Y)$ of the [1] derived equivalence $\Phi$ and look at the images $\Psi\left(\mathcal{O}_{0} \otimes \rho\right) \in D(Y)$ which are supported on the exceptional locus of $Y$. The correspondence $\rho \leftrightarrow \operatorname{Supp} \Psi\left(\mathcal{O}_{0} \otimes \rho\right)$ is what we think of as the geometric McKay correspondence in dimension three since in dimension two this gives the classical McKay correspondence of [6]. a sheaf:

A priori $\Psi\left(\mathcal{O}_{0} \otimes \rho\right)$ will be a complex of sheaves but if $G$ is abelian we show that it is

Theorem 1.1. Let $G \subset \mathrm{SL}_{3}(\mathbb{C})$ be a finite, abelian group. Then, for any irreducible representation $\chi \in \operatorname{Irr}(G)$, the Fourier-Mukai transform $\Psi\left(\mathcal{O}_{0} \otimes \chi\right)$ is a pure sheaf (i.e. some shift of a coherent sheaf).

In Section 2 we explain how to compute $\Psi\left(\mathcal{O}_{0} \otimes \rho\right)$ for any $G \subseteq \mathrm{SL}_{3}(\mathbb{C})$. In Section 6 we show how to explicitly calculate the supports of $\Psi\left(\mathcal{O}_{0} \otimes \rho\right)$ when $G$ is abelian by using an explicit description of $G-\mathrm{Hilb}\left(\mathbb{C}^{3}\right)([4])$ and of the universal family over it ([10]).

Based on such computational evidence, we present the following conjecture as to the exact form of the sheaves $\Psi\left(\mathcal{O}_{0} \otimes \chi\right)$. A part of it gets proven in the course of proving Theorem 1.1.

Conjecture 1.1. Let $G \subset \mathrm{SL}_{3}(\mathbb{C})$ be a finite, abelian group. Then, for any irreducible representation $\chi \in \operatorname{Irr}(G)$, the Fourier-Mukai transform $\Psi\left(\mathcal{O}_{0} \otimes \chi\right)$ is one of the following:

(1) $\mathscr{L}_{\chi}^{-1} \otimes \mathcal{O}_{E_{i}}$,

(2) $\mathscr{L}_{\chi}^{-1} \otimes \mathcal{O}_{E_{i} \cap E_{j}}$,

(3) $\mathscr{F}[1]$ where $\operatorname{Supp}_{Y}(\mathscr{F})=E_{i_{1}} \cup \cdots \cup E_{i_{k}}$,

(4) $\mathcal{O}_{Y}(\operatorname{Exc}(Y)) \otimes \mathcal{O}_{\operatorname{Exc}(Y)}[2]$,

where $E_{i}$ are irreducible exceptional divisors, $\mathscr{F}$ a coherent sheaf and $\mathscr{L}_{\chi}$ is the tautological line bundle of [6].

We expect a similar picture to hold for non-abelian $G \subset \mathrm{SL}_{3}\left(\mathbb{C}^{3}\right)$. In particular:

Conjecture 1.2. For any finite $G \subset \mathrm{SL}_{3}(\mathbb{C})$ the images $\Psi\left(\mathcal{O}_{0} \otimes \chi\right)$ are pure sheaves. 
1.1. An example. Let $G=\frac{1}{13}(1,5,7) \subset \mathrm{SL}_{3}(\mathbb{C})$. For the definition see Section 6 which works out this example in detail. Then $G-\operatorname{Hilb}\left(\mathbb{C}^{3}\right)$ is defined by the toric fan in Figure 1.

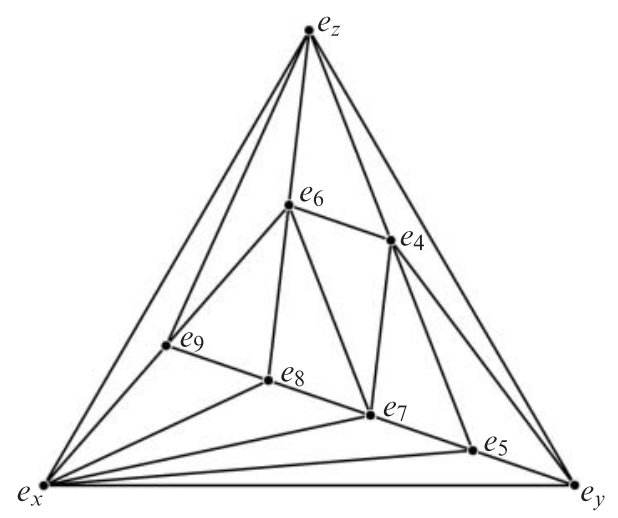

Figure 1. Toric fan of $G-\operatorname{Hilb}\left(\mathbb{C}^{3}\right)$.

Computing the images $\Psi\left(\mathcal{O}_{0} \otimes \chi\right)$ for each $\chi \in \operatorname{Irr}(G)$ we summarise the results in the following table.

\begin{tabular}{|r|r|r|r|}
\hline$\chi$ & $\operatorname{Supp} H^{-2}\left(\Psi\left(\mathcal{O}_{0} \otimes \chi\right)\right)$ & $\operatorname{Supp} H^{-1}\left(\Psi\left(\mathcal{O}_{0} \otimes \chi\right)\right)$ & $\operatorname{Supp} H^{-0}\left(\Psi\left(\mathcal{O}_{0} \otimes \chi\right)\right)$ \\
\hline$\chi_{0}$ & $E_{4} \cup E_{5} \cup E_{6} \cup E_{7} \cup E_{8} \cup E_{9}$ & 0 & 0 \\
$\chi_{1}$ & 0 & 0 & $E_{4}$ \\
$\chi_{2}$ & 0 & 0 & $E_{7}$ \\
$\chi_{3}$ & 0 & $E_{7}$ & 0 \\
$\chi_{4}$ & 0 & 0 & $E_{6}$ \\
$\chi_{5}$ & 0 & 0 & $E_{9}$ \\
$\chi_{6}$ & 0 & $E_{4} \cup E_{6} \cup E_{9}$ & 0 \\
$\chi_{7}$ & 0 & 0 & $E_{5}$ \\
$\chi_{8}$ & 0 & $E_{4} \cup E_{5}$ & 0 \\
$\chi_{9}$ & 0 & 0 & $E_{6} \cap E_{7}$ \\
$\chi_{10}$ & 0 & 0 & $E_{8}$ \\
$\chi_{11}$ & 0 & 0 \\
$\chi_{12}$ & 0 & $E_{5} \cup E_{7} \cup E_{8} \cup E_{9}$ & 0 \\
\hline
\end{tabular}

1.2. Relation to Reid's recipe. From [2], Proposition 9.3, we know that if $\chi$ marks an irreducible exceptional divisor $E$ by Reid's recipe then $\Psi\left(\mathcal{O}_{0} \otimes \chi\right) \cong \mathscr{L}_{\chi}^{-1} \otimes \mathcal{O}_{E}$.

However it is worth noting that in all the examples we computed the supports of $\Psi\left(\mathcal{O}_{0} \otimes \chi\right)$ appear to be more strongly related to Reid's recipe. As an example, we give in Figure 2 the markings according to Reid's recipe for $G=\frac{1}{13}(1,5,7)$ and invite the 
reader to compare it to the data in Table 1.3. For instance, $\Psi\left(\mathcal{O}_{0} \otimes \chi_{12}\right)$ is supported on $E_{5} \cup E_{7} \cup E_{8} \cup E_{9}$ - precisely those divisors which contain two of the curves marked by $\chi_{12}$ by Reid's recipe.

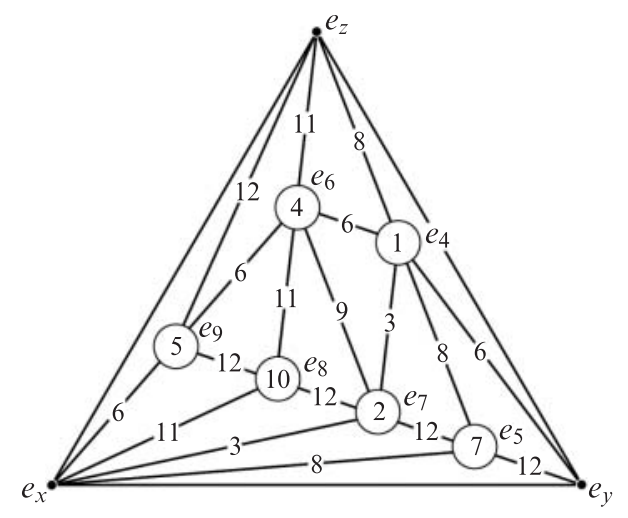

Figure 2. Reid's recipe marking for $G=\frac{1}{13}(1,5,7)$.

We hope to prove the following:

Conjecture 1.3. Let $G \subset \mathrm{SL}_{3}(\mathbb{C})$ be a finite, abelian group. In Reid's recipe,

(1) if $\chi$ marks a divisor then $\Psi\left(\mathcal{O}_{0} \otimes \chi\right)$ is supported in degree 0 on that divisor,

(2) if $\chi$ marks several irreducible curves then $\Psi\left(\mathcal{O}_{0} \otimes \chi\right)$ is supported in degree -1 on the union of all divisors containing two or more of these curves,

(3) if $\chi$ marks a single irreducible curve then $\Psi\left(\mathcal{O}_{0} \otimes \chi\right)$ is supported in degree 0 on that curve.

1.3. Further remarks. (1) Theorem 1.1 does not necessarily hold if you replace $Y=G-\mathrm{Hilb}\left(\mathbb{C}^{3}\right)$ by another crepant resolution of $\mathbb{C}^{3} / G$. For example, let $G=\frac{1}{11}(1,3,7)$ and let $Y$ be the fine module space $M_{\theta}$ of $\theta$-stable $G$-constellations for $\theta=(13,-4,5,-4,-4,1,-4,9,-4,-4,-4)$ (see [2] for more detail). Set $\mathscr{M}$ to be the universal family on $Y \times \mathbb{C}^{3}$. Compute the toric fan of $M_{\theta}$ as described in [3], Section 6, and compute $\mathscr{M}$ as described in [9], Section 4.5. We can then compute $\Psi\left(\mathcal{O}_{0} \otimes \chi_{0}\right)$ just like in Section 6 and it turns out to have non-zero cohomologies both in degree -2 and in degree -1 .

If one examines the proof of Theorem 1.1 then one would find that Propositions 4.5 and 4.12 are the basic results which depend on the fact we are using (the universal family over) $G-\mathrm{Hilb}\left(\mathbb{C}^{3}\right)$ instead of some other crepant resolution.

(2) We prove Theorem 1.1 by doing some very explicit computations with the representation $\mathrm{Q}(G)_{\mathscr{M}}$ of the McKay quiver $\mathrm{Q}(G)$ associated to the universal family $\mathscr{M}$. It would be nice to obtain a more conceptual proof of this result. 
For example, Alexei Bondal suggested the following argument why Theorem 1.1 might hold. The idea is to use his result which states that any exceptional object on a Del-Pezzo surface is a sheaf. On the other hand, there is the following result of Seidel and Thomas. If $i: X \rightarrow Y$ is an inclusion of a divisor satisfying $i^{*} \omega_{Y}=\mathcal{O}_{X}$ then $i_{*} \mathscr{F}$ is spherical if and only if $\mathscr{F}$ is exceptional.

In our case we take $Y=G$-Hilb $\left(\mathbb{C}^{3}\right)$ and pretend that the union $X \subset Y$ of its exceptional divisors is a Del-Pezzo surface. Now $\mathcal{O}_{0} \otimes \chi$ is a spherical object in $D^{G}\left(\mathbb{C}^{3}\right)$ so $\Psi\left(\mathcal{O}_{0} \otimes \chi\right)$ is a spherical object in $Y$. If one could prove directly that $\Psi\left(\mathcal{O}_{0} \otimes \chi\right)$ is the pushforward of an object in $D(X)$ then this object should be a sheaf by Bondal's result. At present, such a direct proof is beyond us. Note that in light of the remark 1 it would need to use the fact that $\mathscr{M}$ is the family of $G$-clusters and not of arbitrary $G$-constellations.

(3) Finally, we see in Section 2 that $\Psi: D^{G}\left(\mathbb{C}^{3}\right) \rightarrow D(Y)$, the inverse of [1] transform $\Phi$, is the transform defined by the dual of the universal family $\mathscr{M}$. We could have instead taken the transform defined by $\mathscr{M}$ itself, as was done in [6]. This choice is only for convenience. In both cases the transforms of $\mathcal{O}_{0} \otimes \chi$ are (shifted) sheaves. However, using our choice the structure sheaves of irreducible divisors appear in degree 0 , while $\Psi\left(\mathcal{O}_{0} \otimes \rho_{0}\right)$ appears in degree -2 and not vice versa. Moreover, this choice matches up better with the marking given by Reid's recipe.

The paper is organized as follows. In Section 2 we describe the transform $\Psi\left(\mathcal{O}_{0} \otimes \rho\right)$ as a cube complex. In Section 3 we compute the cohomology of a general cube complex. In Section 4 we introduce the associated McKay quiver representation and relate it to the cube complex from Section 2. We also prove some properties which it satisfies. In Section 5 we use these properties to prove Theorem 1.1. Finally, in Section 6 we explicitly work out $\Psi\left(\mathcal{O}_{0} \otimes \rho\right)$ when $G=\frac{1}{13}(1,5,7)$.

Acknowledgments. We would like to thank Alexei Bondal, Torsten Ekedahl and Alastair Craw for useful discussions and suggestions. This paper was conceived during the authors' stay at Institut Mittag-Leffler (Sweden) during its "Moduli Spaces" program in 2006-2007. The authors would like to thank the organizers of this program as well as everyone else at the institute for their hospitality. The first author would also like to thank the mathematics department at Rice University. The second author did most of his work on this paper during his stay at KTH (Sweden) and would like to thank them for their support.

\section{The inverse transform and the dual family}

2.1. Notation. In this section, let $G$ be an arbitrary finite subgroup of $\mathrm{SL}_{n}(\mathbb{C})$ and $Y$ a smooth $n$-dimensional separable scheme of finite type over $\mathbb{C}$. We equip $Y$ with the trivial $G$-action. Then $G$ acts naturally on $Y \times \mathbb{C}^{n}$ and we can consider the bounded derived category of $G$-equivariant coherent sheaves $D^{G}\left(Y \times \mathbb{C}^{n}\right)$.

We denote by $V_{\text {giv }}$ the representation of $G$ induced by its inclusion into $\mathrm{SL}_{n}(\mathbb{C})$ and by $R$ the symmetric algebra $S\left(V_{\text {giv }}^{\vee}\right)$. We identify $\mathbb{C}^{n}$ with the affine $G$-scheme $\operatorname{Spec} R$. We also call a $G$-equivariant sheaf a $G$-sheaf for short (cf. [1], Section 4). 
There is an equivalence between the category of quasi-coherent $G$-sheaves on $\mathbb{C}^{n}$ and of $R \rtimes G$-modules which is induced by the functor $\Gamma$ of taking global sections. The relative version of this is an equivalence between the category of quasi-coherent $G$-sheaves on $Y \times \mathbb{C}^{n}$ and of quasi-coherent sheaves of $(R \rtimes G) \otimes_{\mathbb{C}} \mathcal{O}_{Y}$-modules induced by the pushdown functor $\pi_{Y *}$ to $Y$.

For an object $\mathscr{F} \in D^{G}\left(Y \times \mathbb{C}^{n}\right)$ we denote by $\Phi_{\mathscr{F}}$ the integral transform $D(Y) \rightarrow D^{G}\left(\mathbb{C}^{n}\right)$ defined by

$$
\Phi_{\mathscr{F}}(-)=\pi_{\mathbb{C}^{3} *}\left(\mathscr{F} \stackrel{\mathbf{L}}{\otimes} \pi_{Y}^{*}\left(-\otimes \rho_{0}\right)\right)
$$

where $\left(-\otimes \rho_{0}\right)$ is the functor of equipping the sheaf with the trivial $G$-action. And we denote by $\Psi_{\mathscr{F}}$ the integral transform in the opposite direction $D^{G}\left(\mathbb{C}^{n}\right) \rightarrow D(Y)$ defined by

$$
\Psi_{\mathscr{F}}(-)=\pi_{Y *}\left(\mathscr{F} \stackrel{\mathbf{L}}{\otimes} \pi_{\mathbb{C}^{n}}^{*}(-)\right)^{G} .
$$

In brief, a gnat-family is a family on $Y \times \mathbb{C}^{n}$, flat over $Y$, of certain finite-length sheaves on $\mathbb{C}^{n}$ called $G$-constellations, each of which is supported on a single $G$-orbit. Sometimes we will abuse notation by identifying a gnat-family with its pushforward to $Y$ via $\pi_{Y *}$ as an $(R \rtimes G) \otimes_{\mathbb{C}} \mathcal{O}_{Y}$-module. The basic example to keep in mind is the pushforward to $Y=G$-Hilb $\left(\mathbb{C}^{n}\right)$ of the universal family of $G$-clusters. For the precise definition see [9], Section 3.2, and [10].

Suppose $\mathscr{F}$ is a gnat-family on $Y \times \mathbb{C}$. It was shown in [9], Lemma 4 , that the left adjoint to $\Phi_{\mathscr{F}}$ is the integral transform $\Psi_{\mathscr{F} \vee[n]}$. In this section, we show that $\mathscr{F}^{\vee}[n]$ is the gnat-family $\tilde{\mathscr{F}}$ dual to $\mathscr{F}$. Notice that $\tilde{\mathscr{F}}$ is a sheaf (i.e. a complex concentrated in degree zero). We then use this to compute $\Psi_{\mathscr{F} \vee}[n]\left(\mathcal{O}_{0} \otimes \rho\right)$. The application we have in mind is the case $n=3$ with $Y=G$-Hilb $\left(\mathbb{C}^{3}\right)$ and $\mathscr{F}$ the universal family of $G$-clusters on $Y \times \mathbb{C}^{3}$.

2.2. The dual family. If $W$ is an $R \rtimes G$-module, then its dual $W^{\vee}=\operatorname{Hom}_{\mathbb{C}}(W, \mathbb{C})$ is naturally an $R \rtimes G$-module via

$$
g \cdot \alpha(\bullet)=\alpha\left(g^{-1} \cdot \bullet\right) \quad \text { and } \quad m \cdot \alpha(\bullet)=\alpha(m \cdot \bullet)
$$

where $\alpha \in \operatorname{Hom}_{\mathbb{C}}(W, \mathbb{C}), g \in G$ and $m \in R$.

Given a finite-length $G$-sheaf $\mathscr{V}$ on $\mathbb{C}^{n}$ we can define its dual $\mathscr{V}^{\vee}$ to be the $G$-sheaf corresponding to the dual of the $R \rtimes G$-module $\Gamma(\mathscr{V})$. Similarly, if $\mathscr{F} \in \operatorname{Coh}^{G}\left(Y \times \mathbb{C}^{n}\right)$ is a gnat-family then the corresponding family of $R \rtimes G$-modules is $\pi_{Y *} \mathscr{F}$. We define the dual gnat-family $\tilde{\mathscr{F}}$ to be the gnat-family corresponding to $\left(\pi_{*} \mathscr{F}\right)^{\vee}=\operatorname{Hom}_{Y}\left(\pi_{Y *} \mathscr{F}, \mathcal{O}_{Y}\right)$.

Proposition 2.1. If $\mathscr{F}$ is a gnat-family on $Y \times \mathbb{C}^{n}$ then $\mathscr{F} \vee[n] \cong \tilde{\mathscr{F}}$.

Proof. We would like to use Grothendieck-Serre duality for the morphism $\pi_{Y}: Y \times \mathbb{C}^{n} \rightarrow Y$. But $\pi_{Y}$ is manifestly non-proper. However, using Deligne's approach via compactification and pro-categories described in [5] we can still obtain the duality for the full subcategory of $D^{G}\left(Y \times \mathbb{C}^{n}\right)$ consisting of objects whose support is proper over $Y$. Specifically, we get a natural isomorphism

$$
\pi_{Y *} \mathbf{R} \operatorname{Hom}_{Y \times \mathbb{C}^{n}}\left(A, \pi_{Y}^{*} B \times \omega_{Y \times \mathbb{C}^{n} / Y}[n]\right) \stackrel{\sim}{\rightarrow} \mathbf{R} \operatorname{Hom}_{Y}\left(\pi_{Y *} A, B\right)
$$


for any $A \in D^{G}\left(Y \times \mathbb{C}^{n}\right)$ whose support is proper over $Y$ and any $B \in D^{G}(Y)$. Observe that as $\pi_{Y}$ is both flat and affine, $\pi_{Y}^{*}$ and $\pi_{Y *}$ are both exact.

Setting $A=\mathscr{F}$ and $B=\mathcal{O}_{Y}$ yields

$$
\pi_{Y *} \mathscr{F} \vee[n] \stackrel{\sim}{\rightarrow} \mathbf{R} \operatorname{Hom}_{Y}\left(\pi_{Y *} \mathscr{F}, \mathcal{O}_{Y}\right)
$$

since $\omega_{Y \times \mathbb{C}^{n} / Y}$ is trivial. Because the right-hand side is by definition $\pi_{Y *} \tilde{\mathscr{F}}$ the claim follows.

For the skeptical reader unconvinced of the validity of (2.4) we give an alternative proof of Proposition 2.1 in the Appendix. It computes $\mathscr{F}^{\vee}$ by resolving $\mathscr{F}$ by locally free sheaves. To do this we give a natural locally free resolution for finite-length sheaves on $\mathbb{C}^{n}$ that may be of independent interest.

2.3. The image $\Psi\left(\mathcal{O}_{0} \otimes \rho\right) \in \boldsymbol{D}(\boldsymbol{Y})$. Let $\mathcal{O}_{0}$ be the structure sheaf of the origin $0 \in \mathbb{C}^{n}$. For any irreducible representation $\rho \in \operatorname{Irr} G$ denote by $\mathcal{O}_{0} \otimes \rho$ the $G$-sheaf where $\mathcal{O}_{\mathbb{C}^{n}}$ acts on the first factor and $G$ on the second.

The proposition below shows how to compute $\Psi_{\mathscr{F}}\left(\mathcal{O}_{0} \otimes \rho\right)$ for any gnat-family $\mathscr{F}$ on $Y$. In view of Proposition 2.1 we can compute $\Psi_{\mathscr{F} \vee}[n]\left(\mathcal{O}_{0} \otimes \chi\right)$ by setting $\mathscr{F}$ in the proposition below to be the dual family $\tilde{\mathscr{F}}$.

Proposition 2.2. Let $\mathscr{F}$ be any gnat-family over $Y$. The image of $\mathcal{O}_{0} \otimes \rho$ under the integral transform $\Psi_{\mathscr{F}}: D^{G}\left(\mathbb{C}^{n}\right) \rightarrow D(Y)$ is the G-invariant part of the complex

$$
\wedge^{n} V_{\text {giv }}^{\vee} \otimes \pi_{Y *} \mathscr{F} \otimes \rho \stackrel{\delta_{n}}{\longrightarrow} \cdots \stackrel{\delta_{k+1}}{\longrightarrow} \wedge^{k} V_{\text {giv }}^{\vee} \otimes \pi_{Y *} \mathscr{F} \otimes \rho \stackrel{\delta_{k}}{\longrightarrow} \cdots \stackrel{\delta_{1}}{\longrightarrow} \pi_{Y *} \mathscr{F} \otimes \rho
$$

where the rightmost term lies in degree zero. The differentials $\delta_{k}$ are defined for $m_{i} \in V_{\text {giv }}^{\vee}$, $s \in \pi_{Y *} \mathscr{F}$ and $v \in \rho$ by

$$
\left(m_{1} \wedge \cdots \wedge m_{k}\right) \otimes s \otimes v \mapsto \sum_{i=1}^{k}(-1)^{i}\left(\ldots m_{i-1} \wedge m_{i+1} \ldots\right) \otimes\left(m_{i} \cdot s\right) \otimes v
$$

Proof. To compute $\Psi_{\mathscr{F}}\left(\mathcal{O}_{0} \otimes \rho\right)=\pi_{Y *}\left(\mathscr{F} \stackrel{\mathbf{L}}{\otimes}_{Y \times \mathbb{C}^{n}} \pi_{\mathbb{C}^{n}}^{*}\left(\mathcal{O}_{0} \otimes \rho\right)\right)^{G}$ we first resolve $\mathcal{O}_{0} \otimes \rho$ via a Koszul resolution. Observe that elements of $V_{\text {giv }}^{\vee}$ are the non-constant linear functions on $\mathbb{C}^{n}$ and so any basis of $V_{\text {giv }}^{\vee}$ generates the ideal of $0 \in \mathbb{C}^{n}$. We therefore obtain the complex

$$
\wedge^{n} V_{\text {giv }}^{\vee} \otimes \mathcal{O}_{\mathbb{C}^{n}} \otimes \rho \rightarrow \cdots \rightarrow \wedge^{k} V_{\text {giv }}^{\vee} \otimes \mathcal{O}_{\mathbb{C}^{n}} \otimes \rho \rightarrow \cdots \rightarrow \mathcal{O}_{\mathbb{C}^{n}} \otimes \rho
$$

with the differential maps

$$
\left(m_{1} \wedge \cdots \wedge m_{k}\right) \otimes s \otimes v \mapsto \sum_{i=1}^{k}(-1)^{i+1}\left(\ldots m_{i-1} \wedge m_{i+1} \ldots\right) \otimes m_{i} \cdot s \otimes v
$$

where $m_{i} \in V_{\text {giv }}^{\vee}, s \in \mathcal{O}_{\mathbb{C}^{n}}$ and $v \in \rho$. 
Pulling back to $Y \times \mathbb{C}^{n}$ and tensoring with $\mathscr{F}$ we get

$$
\wedge^{n} V_{\text {giv }}^{\vee} \otimes \mathscr{F} \otimes \rho \rightarrow \cdots \rightarrow \wedge^{k} V_{\text {giv }}^{\vee} \otimes \mathscr{F} \otimes \rho \rightarrow \cdots \rightarrow \mathscr{F} \otimes \rho
$$

The result now follows by applying $\pi_{Y *}$.

2.3.1. The abelian case. If the group $G$ is abelian then we can describe the results above more explicitly. This is because we can decompose any gnat-family $\pi_{Y *} \mathscr{F}$ on $Y$ into eigensheaves as follows.

Any gnat-family $\mathscr{F}$ defines a Hilbert-Chow morphism $\pi_{\mathscr{F}}: Y \rightarrow \mathbb{C}^{n} / G$ which sends any $y \in Y$ to the $G$-orbit in $\mathbb{C}^{n}$ that the fiber $\mathscr{F}_{\mid}$is supported on. We can then define the notion of $G$-Weil divisors on $Y$ as in [10], Section 2. Any gnat-family on $Y$ can be decomposed as $\pi_{Y *} \mathscr{F}=\bigoplus_{\chi \in G^{\vee}} \mathscr{L}\left(-D_{\chi}\right)$ for a unique set of $G$-Weil divisors $\left\{D_{\chi}\right\}$ with $D_{\chi_{0}}=0$ (see [10], Section 3.1). A good thing to remember is that $G$ acts on $\mathscr{L}\left(D_{\chi}\right)$ by the character $\chi$ and hence on $\mathscr{L}\left(-D_{\chi}\right)$ by $\chi^{-1}$.

Fix a basis of $G$-eigenvectors for $V_{\text {giv }}^{\vee}$ and denote it by $x_{1}, \ldots, x_{n}$. This choice determines a $G$-eigenvector basis $I_{k}=\left\{x_{i_{1}} \wedge \cdots \wedge x_{i_{k}}: i_{1}<\cdots<i_{k}\right\}$ for each of the spaces $\wedge^{k} V_{\text {giv }}^{\vee}$. Denote by $\kappa\left(x_{i}\right)$ the character with which $G$ acts on $x_{i}$. More generally, given any element $e=x_{i_{1}} \wedge \cdots \wedge x_{i_{k}}$ of $I_{k}$ denote by $\kappa(e)$ the character $\kappa\left(x_{i_{1}}\right) \ldots \kappa\left(x_{i_{k}}\right)$, and similarly for Laurent monomials in $x_{i}$.

We can now give a refinement of Proposition 2.2.

Proposition 2.3. Suppose $G$ is abelian, let $\chi$ be a character of $G$ and let $\mathscr{F}=\bigoplus_{\chi \in G^{\vee}} \mathscr{L}\left(-D_{\chi}\right)$ be a gnat-family. Then $\Psi_{\mathscr{F}}\left(\mathcal{O}_{0} \otimes \chi\right) \in D(Y)$ is equivalent to the complex

$$
\begin{aligned}
\bigoplus_{e \in I_{n}} \mathscr{L}\left(-D_{\kappa(e) \chi}\right) & \rightarrow \cdots \rightarrow \bigoplus_{e \in I_{k}} \mathscr{L}\left(-D_{\kappa(e) \chi}\right) \\
& \rightarrow \cdots \rightarrow \bigoplus_{e \in I_{0}} \mathscr{L}\left(-D_{\kappa(e) \chi}\right) \simeq \mathscr{L}\left(-D_{\chi}\right)
\end{aligned}
$$

where, for each $j \in\{1, \ldots, k\}$, the differential

$$
\mathscr{L}\left(-D_{\kappa\left(x_{i_{1}} \wedge \cdots \wedge x_{i_{k}}\right) \chi}\right) \rightarrow \mathscr{L}\left(-D_{\kappa\left(\ldots x_{i_{j-1}} \wedge x_{i_{j+1}} \ldots\right) \chi}\right)
$$

is given by $s \mapsto(-1)^{j} x_{i_{j}} \cdot s$.

Proof. From decomposition $\mathscr{F}=\bigoplus_{\chi^{\prime} \in G^{\vee}} \mathscr{L}\left(-D_{\chi^{\prime}}\right)$ and $\wedge^{k} V_{\text {giv }}^{\vee}=\bigoplus_{e \in I_{k}} \mathbb{C} e$ we get

$$
\wedge^{k} V_{\mathrm{giv}}^{\vee} \otimes \pi_{Y *} \mathscr{F} \otimes \chi=\underset{\chi^{\prime} \in G^{\vee}, e \in I_{k}}{\bigoplus} \mathbb{C} e \otimes \mathscr{L}\left(-D_{\chi^{\prime}}\right) \otimes \chi
$$

The group $G$ acts on $\mathbb{C} e \otimes \mathscr{L}\left(D_{\chi^{\prime}}\right) \otimes \chi$ by the character $\kappa(e)\left(\chi^{\prime}\right)^{-1} \chi$, so the $G$-invariant part consists of terms where $\chi^{\prime}=\kappa(e) \chi$. This gives (2.11). The claim about the differentials follows immediately from (2.7). 
To compute $\Psi_{\tilde{\mathscr{F}}}\left(\mathcal{O}_{0} \otimes \chi\right)$ we need the result below which allows us to express the $G$ Weil divisors defining the dual family $\tilde{\mathscr{F}}$ in terms of those defining $\mathscr{F}$ :

Proposition 2.4. If $\mathscr{F}=\bigoplus_{\chi \in G^{\vee}} \mathscr{L}\left(-D_{\chi}\right)$ then $\tilde{\mathscr{F}}=\bigoplus_{\chi \in G^{\vee}} \mathscr{L}\left(-D_{\chi}^{\prime}\right)$ where $D_{\chi}^{\prime}=-D_{\chi^{-1}}$.

Proof. For any two $G$-Weil divisors $A$ and $B$ there is a standard $\mathcal{O}_{Y}$-module isomorphism $\mathscr{L}(B-A) \stackrel{\sim}{\rightarrow} \operatorname{Hom}_{\mathcal{O}_{Y}}(\mathscr{L}(A), \mathscr{L}(B))$. The sheaves $\mathscr{L}(A), \mathscr{L}(B)$ and $\mathscr{L}(B-A)$ all come with a natural embedding into the constant sheaf $K\left(\mathbb{C}^{n}\right)$ on $Y$ and the isomorphism sends a section of $\mathscr{L}(B-A)$ to the map of the multiplication by that section inside of $K\left(\mathbb{C}^{n}\right)$.

In particular, this gives an $\mathcal{O}_{Y}$-module isomorphism

$$
\phi: \bigoplus_{\chi \in G^{\vee}} \mathscr{L}\left(D_{\chi}\right) \stackrel{\sim}{\rightarrow} \operatorname{Hom}_{\mathcal{O}_{Y}}\left(\bigoplus_{\chi \in G^{\vee}} \mathscr{L}\left(-D_{\chi}\right), \mathcal{O}_{Y}\right)=\tilde{\mathscr{F}}
$$

We claim that $\phi$ is $R \rtimes G$-equivariant, thus making it an isomorphism of $\mathcal{O}_{Y} \otimes(R \rtimes G)$ modules. Let $s$ be a section of $\bigoplus_{\chi \in G^{\vee}} \mathscr{L}\left(D_{\chi^{-1}}\right)$ and $t$ a section of $\bigoplus_{\chi \in G^{\vee}} \mathscr{L}\left(-D_{\chi}\right)$. Then for
any $g \in G$ and $m \in R$

$$
\begin{aligned}
& \phi(r \cdot s)(t)=r s t=r \cdot(\phi(s)(t))=(r \cdot \phi(s))(t) \\
& \phi(g \cdot s)(t)=(g \cdot s) t=g \cdot\left(s\left(g^{-1} \cdot t\right)\right) \stackrel{(1)}{=} s\left(g^{-1} \cdot t\right)=\phi(s)\left(g^{-1} \cdot t\right)=(g \cdot \phi(s))(t)
\end{aligned}
$$

where the equality (1) is due to the fact that $s\left(g^{-1} \cdot t\right)$ is an element of $\mathcal{O}_{Y} \subseteq K\left(\mathbb{C}^{n}\right)$ and thus $G$-invariant. Finally, since $G$ acts by $\chi$ on $\mathscr{L}\left(D_{\chi}\right)$ it must be the summand $\mathscr{L}\left(-D_{\chi^{-1}}^{\prime}\right)$ of $\tilde{\mathscr{F}}$. This yields $D_{\chi}^{\prime}=-D_{\chi^{-1}}$. Corollary 2.5. Suppose $G$ is abelian, $\chi$ is a character of $G$ and let $\mathscr{F}=\bigoplus_{\chi \in G^{\vee}} \mathscr{L}\left(-D_{\chi}\right)$
be a gnat-family. Then $\Psi_{\tilde{\mathscr{F}}}\left(\mathcal{O}_{0} \otimes \chi\right) \in D(Y)$ is equivalent to the complex

$$
\begin{aligned}
\bigoplus_{e \in I_{n}} \mathscr{L}\left(D_{\kappa(e)^{-1} \chi^{-1}}\right) & \rightarrow \cdots \rightarrow \bigoplus_{e \in I_{k}} \mathscr{L}\left(D_{\kappa(e)^{-1} \chi^{-1}}\right) \\
& \rightarrow \cdots \rightarrow \bigoplus_{e \in I_{0}} \mathscr{L}\left(D_{\kappa(e)^{-1} \chi^{-1}}\right) \simeq \mathscr{L}\left(D_{\chi^{-1}}\right)
\end{aligned}
$$

where, for each $j \in\{1, \ldots, k\}$, the differential

$$
\mathscr{L}\left(D_{\kappa\left(x_{i_{1}} \wedge \cdots \wedge x_{i_{k}}\right)^{-1} \chi^{-1}}\right) \rightarrow \mathscr{L}\left(D_{\kappa\left(\ldots x_{i_{j-1}} \wedge x_{i_{j+1}} \ldots\right)^{-1} \chi^{-1}}\right)
$$

is given by $s \mapsto(-1)^{j} x_{i_{j}} \cdot s$.

2.4. Examples. We end this section with a couple of examples.

Example 2.6. Let $G$ be the group $\frac{1}{7}(1,6)$. That is, $G \cong \mathbb{Z} / 7 \mathbb{Z}$ embedded in $\mathrm{SL}_{2}(\mathbb{C})$ via $1 \mapsto\left(\begin{array}{cc}\xi & \\ & \xi^{6}\end{array}\right)$. Denote by $\chi_{i}$ the character of $G$ given by $i \mapsto \xi^{i}$. In these 
terms, $\kappa\left(x_{1}\right)=\chi_{6}$ and $\kappa\left(x_{2}\right)=\chi_{1}$. Let $Y$ be the minimal resolution of $\mathbb{C}^{2} / G$ and let $\mathscr{F}=\bigoplus \mathscr{L}\left(-D_{\chi}\right)$ be any gnat-family on $Y$. Then $\Psi_{\tilde{F}}\left(\mathcal{O}_{0} \otimes \chi_{3}\right) \in D(Y)$ is given by the (total complex of the) square

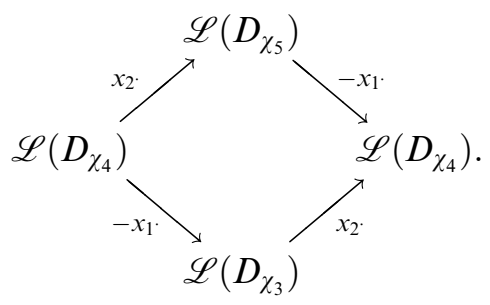

By convention the rightmost term is sitting in degree 0 .

Example 2.7. Let $G$ be the subgroup $\frac{1}{13}(1,5,7), Y$ a crepant resolution of $\mathbb{C}^{3} / G$ and $\mathscr{F}=\bigoplus \mathscr{L}\left(-D_{\chi}\right)$ a gnat-family on $Y$. Then the $\Psi_{\tilde{F}}\left(\mathcal{O}_{0} \otimes \chi_{5}\right) \in D(Y)$ is given by the (total complex of the) cube

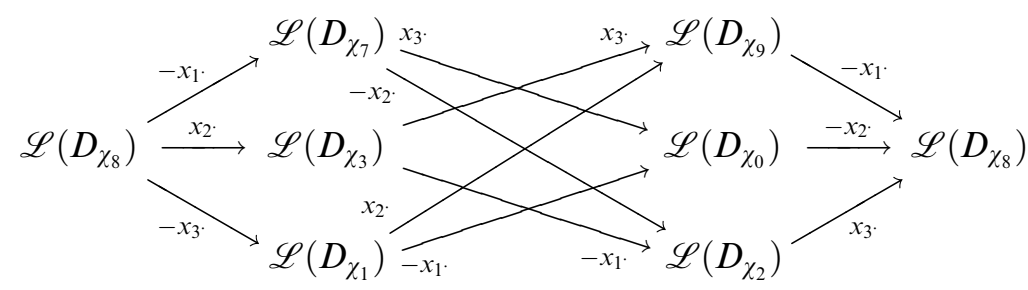

where, again, the rightmost term is sitting in degree 0 .

\section{Cohomology of skew-commutative cubes}

In this section we study the cohomology of abstract complexes of the form (2.15) with the aim to use this later to calculate the cohomology sheaves of $\Psi\left(\mathcal{O}_{0} \otimes \chi\right)$.

Let $X$ be a smooth separated scheme over $\mathbb{C}$. Let $S:=\{1, \ldots, n\}$ and denote by $V$ the set of subsets of $S$. We identify $V$ with the vertices of an ( $n$-dimensional) unit cube.

By a cube of line bundles we mean the data of a line bundle $\mathscr{L}_{v}$ on $X$ for every vertex $v \in V$ and a morphism $\alpha_{v}^{i}: \mathscr{L}_{v \cup i} \rightarrow \mathscr{L}_{v}$ for each edge $v \cup\{i\} \rightarrow v$ of the cube. One can think of it as a representation of a cube-shaped quiver with vertices $V$ and arrows $v \cup\{i\} \rightarrow v$ into a graded sheaf $\bigoplus_{v \in V} \mathscr{L}_{v}$ over $X$.

We say that a cube is skew-commutative (resp. commutative), if each of its twodimensional faces forms an anti-commutative (resp. commutative) square, i.e.

$$
\alpha_{v}^{i} \circ \alpha_{v \cup i}^{j}+\alpha_{v}^{j} \circ \alpha_{v \cup j}^{i}=0 \quad \text { or } \quad \alpha_{v}^{i} \circ \alpha_{v \cup i}^{j}-\alpha_{v}^{j} \circ \alpha_{v \cup j}^{i}=0,
$$

respectively, for all $v \in V$ with $i, j \notin v$. Given a skew-commutative cube, we can form its total chain complex $T^{\bullet}$ where $T^{-i}=\underset{|I|=i}{\bigoplus} \mathscr{L}_{I}$. The differential $d$ is given by summing the 
maps in the cube. A commutative cube can be turned into a skew-commutative cube by sprinkling some minus signs as follows:

$$
\alpha_{\left\{i_{1} \leqq \cdots \leqq i_{k}\right\}}^{i_{j}} \leadsto(-1)^{j} \alpha_{\left\{i_{1} \leqq \cdots \leqq i_{k}\right\}}^{i_{j}}
$$

We now turn to the case $n=3$ and further assume that the maps $\alpha_{v}^{i}$ are nonzero. The vanishing locus of $\alpha_{v}^{i}$ is then an effective Weil divisor on $X$ which we denote by $D_{v}^{i}$. Note that $\mathcal{O}_{X}\left(D_{v}^{i}\right) \cong \mathscr{L}_{v} \otimes \mathscr{L}_{v \cup i}^{\vee}$. The corresponding skew-commutative cube looks like

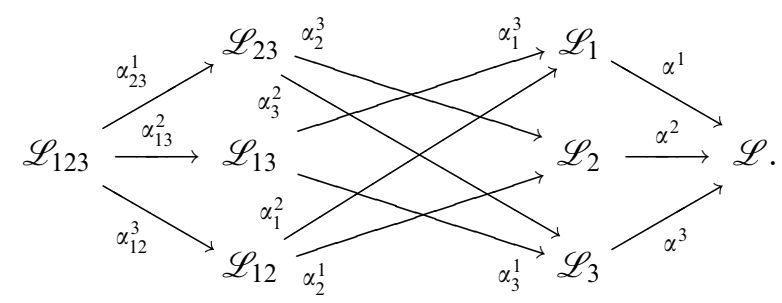

Lemma 3.1. Let $T^{\bullet}$ be the total complex of the skew-commutative cube $\{\mathscr{L}, \alpha\}$ depicted above. Then:

(1) $H^{0}\left(T^{\bullet}\right) \cong \mathscr{L} \otimes \mathcal{O}_{Z}$ where $Z$ is the scheme theoretic intersection $D^{1} \cap D^{2} \cap D^{3}$.

(2) $H^{-1}\left(T^{\bullet}\right)$ has a three step filtration with successive quotients:

- $\mathcal{O}_{Z} \otimes \mathscr{L}_{12}\left(\operatorname{gcd}\left(D_{1}^{2}, D_{2}^{1}\right)\right)$ where $Z$ is the scheme theoretic intersection of $\operatorname{gcd}\left(D_{1}^{2}, D_{2}^{1}\right)$ and the effective part of $D^{3}+\operatorname{cm}\left(\tilde{D}_{3}^{1}, \tilde{D}_{3}^{2}\right)-\tilde{D}_{1}^{2}-D^{1}$,

- $\mathcal{O}_{Z} \otimes \mathscr{L}_{13}\left(\operatorname{gcd}\left(D_{1}^{3}, D_{3}^{1}\right)\right)$ where $Z$ is the scheme theoretic intersection of $\operatorname{gcd}\left(D_{1}^{3}, D_{3}^{1}\right)$ and the effective part of $D^{2}+\operatorname{lcm}\left(D_{2}^{1}, \tilde{D}_{2}^{3}\right)-\tilde{D}_{3}^{1}-D^{3}$,

- $\mathcal{O}_{Z} \otimes \mathscr{L}_{23}\left(\operatorname{gcd}\left(D_{2}^{3}, D_{3}^{2}\right)\right)$ where $Z$ is the scheme theoretic intersection of $\operatorname{gcd}\left(D_{2}^{3}, D_{3}^{2}\right)$ and the effective part of $D^{1}+\operatorname{lcm}\left(D_{1}^{2}, D_{1}^{3}\right)-\tilde{D}_{2}^{3}-D^{2}$,

where $\tilde{D}_{j}^{i}=D_{j}^{i}-\operatorname{gcd}\left(D_{j}^{i}, D_{i}^{j}\right)$.

(3) $H^{-2}\left(T^{\bullet}\right) \cong \mathscr{L}_{123}(D) \otimes \mathcal{O}_{D}$ where $D=\operatorname{gcd}\left(D_{23}^{1}, D_{13}^{2}, D_{12}^{3}\right)$.

(4) $H^{-3}\left(T^{\bullet}\right) \cong 0$.

Proof. We shall need to work locally. Let $p$ be an arbitrary point of $X$. We fix a local generator for each $\mathscr{L}_{e}$, identifying it with $\mathcal{O}_{X, p}$. Each map $\alpha_{e}^{i}$ becomes then an endomorphism of $\mathcal{O}_{X, p}$, i.e. the multiplication by some $f_{e}^{i} \in \mathcal{O}_{X, p}$. Then $f_{e}^{i}$ is a local generator of $D_{e}^{i}$, and we use it to identify $\mathcal{O}_{X}\left(-D_{e}^{i}\right)$ and $\mathcal{O}_{X}\left(D_{e}^{i}\right)$ with $\mathcal{O}_{X, p}$.

(1) We can view $H^{0}\left(T^{\bullet}\right)$ as the cokernel of $\bigoplus_{i} \mathcal{O}_{X}\left(-D^{i}\right) \otimes \mathscr{L} \hookrightarrow \mathcal{O}_{X} \otimes \mathscr{L}$, which is by definition $\mathcal{O}_{Z} \otimes \mathscr{L}$ where $Z=\bigcap_{i} D^{i}$. 
(2) Assume without loss of generality that $D^{1}, D^{2}, D^{3}$ have no prime divisor in common. We claim that the kernel of $d: T^{-1} \rightarrow T^{0}$ is generated by $\beta_{1}=\frac{1}{h^{12}}\left(f^{2}, f^{1}, 0\right)$, $\beta_{2}=\frac{1}{h^{13}}\left(f^{3}, 0, f^{1}\right)$ and $\beta_{3}=\frac{1}{h^{23}}\left(0, f^{3}, f^{2}\right)$ where $h^{i j}=\operatorname{gcd}\left(f^{i}, f^{j}\right)$. Suppose

$$
a f^{1}+b f^{2}+c f^{3}=0
$$

We must have $\operatorname{gcd}\left(\frac{f^{2}}{h^{12}}, \frac{f^{3}}{h^{13}}\right)$ divides $a$, for it divides $f_{1} a$ and no prime divisor of it can divide $f_{1}$ by definitions of $h^{12}$ and $h^{13}$. Thus we can obtain $a$ as a linear combination of $\frac{f^{2}}{h^{12}}$ and $\frac{f^{3}}{h^{13}}$, i.e. we can combine $\beta_{1}$ and $\beta_{2}$ to get an element of the form $\left(a, b^{\prime}, c^{\prime}\right)$. Then $\left(0, b^{\prime}-b, c^{\prime}-c\right)$ is a multiple of $\beta_{3}$ - for it must also lie in the kernel of $d: T^{-1} \rightarrow T^{0}$, which means $f^{2}\left(b^{\prime}-b\right)+f^{3}\left(c^{\prime}-c\right)=0$. This shows the claim.

For any $i \neq j$ denote by $g^{i j}$ the greatest common divisor of $f_{j}^{i}$ and $f_{i}^{j}$ and let $\tilde{f}_{j}^{i}=f_{j}^{i} / g^{i j}$. By skew-commutativity of the cube $f_{i}^{j} f^{i}+f_{j}^{i} f^{j}=0$. Therefore $\tilde{f}_{j}^{i}=f^{i} / h^{i j}$. We now rewrite $\beta_{1}=\left(\tilde{f}_{1}^{2}, \tilde{f}_{2}^{1}, 0\right), \beta_{2}=\left(\tilde{f}_{1}^{3}, 0, \tilde{f}_{3}^{1}\right)$ and $\beta_{3}=\left(0, \tilde{f}_{2}^{3}, \tilde{f}_{3}^{2}\right)$. On the other hand, the image of $d$ in $T^{1}$ is generated by $\left(f_{1}^{2}, f_{2}^{1}, 0\right),\left(f_{1}^{3}, 0, f_{3}^{1}\right)$ and $\left(0, f_{2}^{3}, f_{3}^{2}\right)$. In particular, this means that if $D_{i}^{j}$ and $D_{j}^{i}$ have no common divisors for any $i \neq j$ then $H^{-1}\left(T^{\bullet}\right)=0$.

Consider the filtration $\operatorname{Im}(d)=F^{0} \subset F^{1} \subset F^{2} \subset F^{3}=\operatorname{ker}(d)$ inside $T^{-1}$ where

- $F^{0}$ is generated by $g^{12} \cdot\left(\tilde{f}_{1}^{2}, \tilde{f}_{2}^{1}, 0\right), g^{13} \cdot\left(\tilde{f}_{1}^{3}, 0, \tilde{f}_{3}^{1}\right)$ and $g^{23} \cdot\left(0, \tilde{f}_{2}^{3}, \tilde{f}_{3}^{2}\right)$,

- $F^{1}$ is generated by $g^{12} \cdot\left(\tilde{f}_{1}^{2}, \tilde{f}_{2}^{1}, 0\right), g^{13} \cdot\left(\tilde{f}_{1}^{3}, 0, \tilde{f}_{3}^{1}\right)$ and $\left(0, \tilde{f}_{2}^{3}, \tilde{f}_{3}^{2}\right)$,

- $F^{2}$ is generated by $g^{12} \cdot\left(\tilde{f}_{1}^{2}, \tilde{f}_{2}^{1}, 0\right),\left(\tilde{f}_{1}^{3}, 0, \tilde{f}_{3}^{1}\right)$ and $\left(0, \tilde{f}_{2}^{3}, \tilde{f}_{3}^{2}\right)$,

- $F^{3}$ is generated by $\left(\tilde{f}_{1}^{2}, \tilde{f}_{2}^{1}, 0\right),\left(\tilde{f}_{1}^{3}, 0, \tilde{f}_{3}^{1}\right)$ and $\left(0, \tilde{f}_{2}^{3}, \tilde{f}_{3}^{2}\right)$.

We show that globally the quotient $F^{2} / F^{1}$ is isomorphic to $\mathcal{O}_{Z} \otimes \mathscr{L}_{13}\left(\operatorname{gcd}\left(D_{1}^{3}, D_{3}^{1}\right)\right)$, where $Z$ is the intersection of $\operatorname{gcd}\left(D_{1}^{3}, D_{3}^{1}\right)$ and the effective part of $D^{2}+\operatorname{lcm}\left(D_{2}^{1}, \tilde{D}_{2}^{3}\right)-\tilde{D}_{3}^{1}-D^{3}$ (the other two quotients are computed similarly).

We can combine $g^{12} \cdot\left(\tilde{f}_{1}^{2}, \tilde{f}_{2}^{1}, 0\right)=\left(f_{1}^{2}, f_{2}^{1}, 0\right)$ and $\left(0, \tilde{f}_{2}^{3}, \tilde{f}_{3}^{2}\right)$ to give a multiple of $\left(\tilde{f}_{1}^{3}, 0, \tilde{f}_{3}^{1}\right)$. The smallest such multiple is

$$
\frac{\tilde{f}_{2}^{3}}{\operatorname{gcd}\left(f_{2}^{1}, \tilde{f}_{2}^{3}\right)}\left(f_{1}^{2}, f_{2}^{1}, 0\right)-\frac{f_{2}^{1}}{\operatorname{gcd}\left(f_{2}^{1}, \tilde{f}_{2}^{3}\right)}\left(0, \tilde{f}_{2}^{3}, \tilde{f}_{3}^{2}\right)=\frac{\tilde{f}_{3}^{2} \cdot f_{2}^{1}}{\tilde{f}_{3}^{1} \cdot \operatorname{gcd}\left(f_{2}^{1}, \tilde{f}_{2}^{3}\right)}\left(\tilde{f}_{1}^{3}, 0,-\tilde{f}_{3}^{1}\right) .
$$

Let $h$ be the regular part of $\frac{\tilde{f}_{3}^{2} \cdot f_{2}^{1}}{\tilde{f}_{3}^{1} \cdot \operatorname{gcd}\left(f_{2}^{1}, \tilde{f}_{2}^{3}\right)}$, it is well defined since $\mathcal{O}_{X, p}$ is UFD. Then, locally, $F^{2} / F^{1}$ is $\mathcal{O}_{X, p} /\left(g^{13}, h\right) \cdot\left(\tilde{f}_{1}^{3}, 0, \tilde{f}_{3}^{1}\right)$. Consider now the global injection

$$
\mathscr{L}_{13}\left(\operatorname{gcd}\left(D_{1}^{3}, D_{3}^{1}\right)\right) \stackrel{\alpha_{1}^{3} \oplus 0 \oplus \alpha_{3}^{1}}{\longrightarrow} \mathscr{L}_{1} \oplus \mathscr{L}_{2} \oplus \mathscr{L}_{3} .
$$


Locally, its image in $\mathscr{L}_{1} \oplus \mathscr{L}_{2} \oplus \mathscr{L}_{3}$ is just $\mathcal{O}_{X, p} \cdot\left(\tilde{f}_{1}^{3}, 0, \tilde{f}_{3}^{1}\right)$. It therefore only remains to show that the ideal $\left(g^{13}, h\right)$ defines $Z$ in $\mathcal{O}_{X, p}$. This follows since $\left(g^{13}\right)$ defines $\operatorname{gcd}\left(D_{3}^{1}, D_{1}^{3}\right)$ and $(h)$ - the effective part of

$$
\begin{aligned}
\tilde{D}_{3}^{2}+D_{2}^{1}-\tilde{D}_{3}^{1}-\operatorname{gcd}\left(D_{2}^{1}, \tilde{D}_{2}^{3}\right) & =\tilde{D}_{2}^{3}-D^{3}+D^{2}+D_{2}^{1}-\operatorname{gcd}\left(D_{2}^{1}, \tilde{D}_{2}^{3}\right)-\tilde{D}_{3}^{1} \\
& =D^{2}+\operatorname{lcm}\left(D_{2}^{1}, \tilde{D}_{2}^{3}\right)-D^{3}-\tilde{D}_{3}^{1},
\end{aligned}
$$

where the first equality follows since $\tilde{D}_{3}^{2}+D^{3}=\tilde{D}_{2}^{3}+D^{2}$ by the skew-commutativity.

(3) Suppose, at first, that $D_{23}^{1}, D_{13}^{2}$ and $D_{12}^{3}$ have no divisor in common. We claim that, locally, if $(a, b, c) \in T^{-2}$ lies in the kernel of $d$ then it lies in $d\left(T^{-3}\right)$. Let $g$ be a prime factor of $f_{23}^{1}$ and suppose (without loss of generality) that $g$ does not divide $f_{12}^{3}$. Let $p, q$ be the largest non-negative integers such that $g^{p} \mid f_{23}^{1}$ and $g^{q} \mid f_{2}^{3}$. Commutativity of faces implies $f_{23}^{1} \cdot f_{2}^{3}=f_{12}^{3} \cdot f_{2}^{1}$ so $g^{p+q}$ divides $f_{2}^{1}$. But since $(a, b, c)$ lies in the kernel $a f_{2}^{3}-c f_{2}^{1}=0$. This means $g^{p+q}$ divides $a f_{2}^{3}$. Since the local ring is a UFD we get $g^{p} \mid a$. Since this is true for any $g$ we get $f_{23}^{1} \mid a$. A similar argument shows $f_{13}^{2} \mid b$ and $f_{12}^{3} \mid c$ so $(a, b, c)$ lies in the image of $d$.

More generally, if $D$ is the largest common divisor, then we can factor $d: T^{-3} \rightarrow T^{-2}$ as $\mathscr{L}_{123} \rightarrow \mathscr{L}_{123}(D)$ followed by a map which has no common divisors. By the above, the image of $\mathscr{L}_{123}(D)$ under this map equals the kernel of $d: T^{-2} \rightarrow T^{-1}$. Thus $H^{-2}\left(T^{\bullet}\right)$ is precisely the cokernel of $\mathscr{L}_{123} \hookrightarrow \mathscr{L}_{123}(D)$ which is $\mathcal{O}_{D} \otimes \mathscr{L}_{123}(D)$.

(4) Since any non-zero map between line bundles is injective $\mathscr{L}_{123}$ injects into (say) $\mathscr{L}_{12}$ so $H^{-3}\left(T^{\bullet}\right) \cong 0$.

Lemma 3.1 makes it clear that given a gnat-family $\mathscr{F}=\bigoplus \mathscr{L}\left(-D_{\chi}\right)$ in order to calculate the cohomology sheaves of $\Psi_{\mathscr{F}}\left(\mathcal{O}_{0} \otimes \chi\right)$ we need to calculate the vanishing loci $D_{v}^{i}$ of all maps $\alpha_{v}^{i}$ in all the corresponding skew-commutative cubes. By Proposition 2.3 this amounts to calculating the vanishing loci of maps

$$
\begin{aligned}
\mathscr{L}\left(-D_{\chi}\right) & \rightarrow \mathscr{L}\left(-D_{\chi \kappa\left(x_{i}\right)^{-1}}\right), \\
s & \mapsto x_{i} \cdot s,
\end{aligned}
$$

for all $\chi$ and $x_{i}$. We learn to do this in our next section.

\section{The associated representation of the McKay quiver}

In this section, we take $G$ to be a finite abelian subgroup of $\mathrm{SL}_{3}(\mathbb{C}), Y \rightarrow \mathbb{C}^{3} / G$ to be any crepant resolution and $\mathscr{F}=\bigoplus \mathscr{L}\left(-D_{\chi}\right)$ to be any gnat-family on $Y$. We fix an exceptional divisor $E \in \operatorname{Exc}(Y)$ and generically along $E$ study the behavior of the McKay quiver representation $\mathrm{Q}(G)_{\mathscr{F}}$.

4.1. The McKay quiver of $G \subset \mathrm{SL}_{3}(\mathbb{C})$ and its planar embedding. By a quiver we mean a vertex set $Q_{0}$, an arrow set $Q_{1}$ and a pair of maps $h: Q_{1} \rightarrow Q_{0}$ and $t: Q_{1} \rightarrow Q_{0}$ giving the head $h q \in Q_{0}$ and the tail $t q \in Q_{0}$ of each arrow $q \in Q_{1}$. 
Definition 4.1. The McKay quiver $\mathrm{Q}(G)$ of (a not necessarily abelian) $G \subset \mathrm{SL}_{3}(\mathbb{C})$ is the quiver whose vertex set $Q_{0}$ are the irreducible representations $\rho$ of $G$ and whose arrow set $Q_{1}$ has $\operatorname{dim} \operatorname{Hom}_{G}\left(\rho_{i} \otimes V_{\text {giv }}^{\vee}, \rho_{j}\right)$ arrows going from the vertex $\rho_{i}$ to the vertex $\rho_{j}$.

As we assumed $G$ to be abelian $V_{\text {giv }}^{\vee} \cong \bigoplus \mathbb{C} x_{i}$ as $G$-representations. Let $\chi_{i}$ and $\chi_{j}$ be any pair of characters of $G$. We then see that $\operatorname{Hom}_{G}\left(\chi_{i} \otimes V_{\text {giv }}^{\vee}, \chi_{j}\right)$ contains a copy of $\mathbb{C}$ for each $k \in\{1,2,3\}$ satisfying $\chi_{j}=\kappa\left(x_{k}\right) \chi_{i}$. Thus each vertex $\chi \in G^{\vee}$ of $\mathrm{Q}(G)$ has 3 arrows emerging from it and going to vertices $\kappa\left(x_{k}\right) \chi$ for $k=1,2,3$. We denote the arrow from $\chi$ to $\kappa\left(x_{k}\right) \chi$ by $(\chi, k)$ and say further that it is an $x_{k}$-arrow. For an example see Section 6.1.

The fact that $G \subseteq \mathrm{SL}_{3}(\mathbb{C})$ allows us to embed its McKay quiver in a two dimensional real torus. We briefly recall this embedding as constructed by Craw and Ishii in [2].

Consider the maximal torus $\left(\mathbb{C}^{*}\right)^{3} \subset \mathrm{SL}_{3}(\mathbb{C})$ containing $G$. We have an exact sequence of abelian groups:

$$
0 \rightarrow G \rightarrow\left(\mathbb{C}^{*}\right)^{3} \rightarrow T \rightarrow 0
$$

where $T$ is the quotient torus which acts on the quotient space $\mathbb{C}^{3} / G$. By applying $\operatorname{Hom}\left(\bullet, \mathbb{C}^{*}\right)$ to $(4.1)$ we obtain an exact sequence

$$
0 \rightarrow M \rightarrow \mathbb{Z}^{3} \stackrel{\rho}{\rightarrow} G^{\vee} \rightarrow 0
$$

where $\mathbb{Z}^{3}$ is thought of as the lattice of exponents of Laurent monomials. Given $m=\left(k_{1}, k_{2}, k_{3}\right) \in \mathbb{Z}^{3}$ we write $x^{m}$ for $x_{1}^{k_{1}} x_{2}^{k_{2}} x_{3}^{k_{3}}$. Note that $\rho$ is the weight map, that is $x^{m}(g \cdot \boldsymbol{v})=\rho(m)(g) x^{m}(\boldsymbol{v})$ for any $\boldsymbol{v} \in \mathbb{C}^{3}$. By definition $g \cdot x^{m}(\boldsymbol{v})=x^{m}\left(g^{-1} \cdot \boldsymbol{v}\right)$, so $\rho=\kappa^{-1}$, where $\kappa$ is the map introduced in Section 2.3.1, which maps every Laurent monomial to the character $G$ acts on it with. $M$ is then the sublattice in $\mathbb{Z}^{3}$ of (exponents of) $G$-invariant Laurent monomials. As $G \subseteq \mathrm{SL}_{3}(\mathbb{C})$ we have $(1,1,1) \in M$, i.e. $x_{1} x_{2} x_{3}$ is an invariant monomial. Take $H=\mathbb{Z}^{3} / \mathbb{Z}(1,1,1)$ and $M^{\prime}=M / \mathbb{Z}(1,1,1)$. Then (4.2) induces

$$
0 \rightarrow M^{\prime} \rightarrow H \rightarrow G^{\vee} \rightarrow 0
$$

For every Laurent monomial $x^{m}$ for some $m \in \mathbb{Z}^{3}$ we shall write $\left[x^{m}\right]$ for the class of $m$ in $H$, e.g. $\left[x_{1} x_{2}^{2}\right]$ for the class of $(1,2,0)$.

Definition 4.2. The universal cover $U$ of $\mathrm{Q}(G)$ is the quiver whose vertex set are the elements of $H$ and whose arrow set is

$$
\left\{\left(h, h+\left[x_{i}\right]\right) \mid h \in H, i \in 1,2,3\right\} .
$$

We have a natural 'embedding' of $U$ into $H \otimes \mathbb{R} \cong \mathbb{R}^{2}$, where the arrow $\left(h, h+\left[x_{i}\right]\right)$ is identified with the line segment $\left\{h+\lambda\left[x_{i}\right] \mid \lambda \in(0,1)\right\}$. For illustrative purposes, we fix a specific isomorphism:

$$
\phi_{H}: H \otimes \mathbb{R} \rightarrow \mathbb{R}^{2}:\left\{\begin{array}{l}
{\left[x_{1}\right] \mapsto\left(\frac{\sqrt{3}}{2},-\frac{1}{2}\right),} \\
{\left[x_{3}\right] \mapsto(0,1) .}
\end{array}\right.
$$


As shown in Figure 3 the image of $U$ under $\phi_{H}$ is a tessellation of $\mathbb{R}^{2}$ into regular triangles. The quotient $T_{G}=H \otimes \mathbb{R} / M^{\prime}$ is a two dimensional real torus. $\mathrm{Q}(G)$ is the quotient of $U$ by the action of $M^{\prime}$ and thus is a tessellation of $T_{G}$ into regular triangles. The boundary of a triangle is the cycle

$$
\chi \stackrel{x_{\sigma(1)}}{\longrightarrow} \kappa\left(x_{\sigma(1)}\right) \chi \stackrel{x_{\sigma(2)}}{\longrightarrow} \kappa\left(x_{\sigma(1)} x_{\sigma(2)}\right) \chi \stackrel{x_{\sigma(3)}}{\longrightarrow} \kappa\left(x_{\sigma(1)} x_{\sigma(2)} x_{\sigma(3)}\right) \chi=\chi
$$

where $\sigma \in S_{3}$ is a permutation. There are altogether $2|G|$ triangles in $\mathrm{Q}(G)$. When depicting $T_{G}$ in diagrams we draw its fundamental domain in $\mathbb{R}^{2}$. The depiction of the McKay quiver of $G=1 / 13(1,5,7)$, as embedded into $T_{G}$, is illustrated in Figure 27 (Section 6.1).

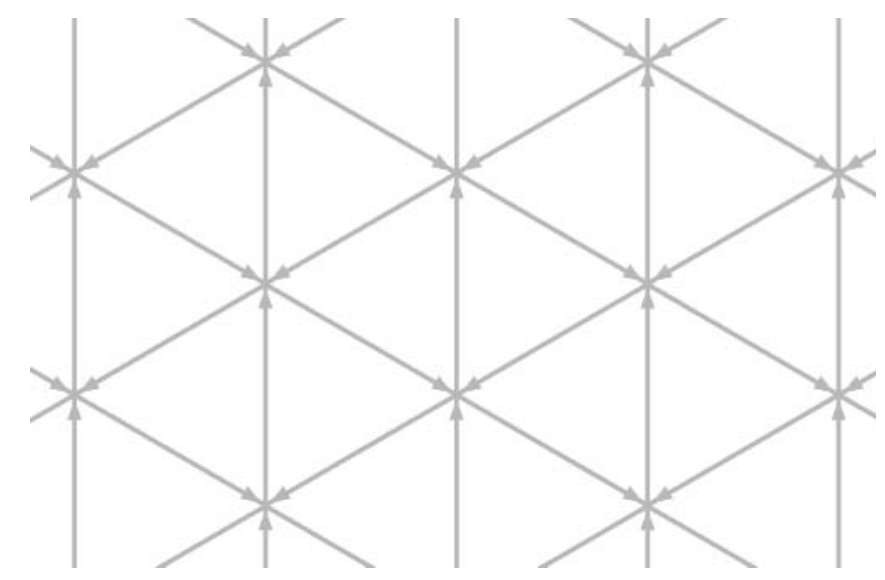

Figure 3. Tessellation of $\mathbb{R}^{2}$ by $U$.

4.2. The associated representation $Q(G)_{\mathscr{F}}$ of the McKay quiver. A representation of a quiver over $\mathbb{C}$ is a graded vector space $\bigoplus_{i \in Q_{0}} V_{i}$ together with a collection of linear maps $\left\{\alpha_{q}: V_{t q} \rightarrow V_{h q}\right\}_{q \in Q_{1}}$. Over an arbitrary scheme $S$ this translates into a graded locally free sheaf $\bigoplus_{i \in Q_{0}} \mathscr{N}_{i}$ and a collection of morphisms $\left\{\alpha_{q}: \mathscr{N}_{t q} \rightarrow \mathscr{N}_{h q}\right\}_{q \in Q_{1}}$.

Definition 4.3. Given a gnat-family $\mathscr{F}=\bigoplus_{\chi \in G^{\vee}} \mathscr{L}\left(-D_{\chi}\right)$ on $Y$ the associated representation $\mathrm{Q}(G)_{\mathscr{F}}$ of the McKay quiver over $Y$ is the sheaf $\mathscr{F}$ where the summand $\mathscr{L}\left(-D_{\chi^{-1}}\right)$ is graded by $\chi$ (since $G$ acts by $\chi$ on $\mathscr{L}\left(-D_{\chi^{-1}}\right)$ ). The maps $\alpha_{\chi, x_{k}}$ are defined by

$$
\alpha_{\chi, x_{k}}: \mathscr{L}\left(-D_{\chi^{-1}}\right) \rightarrow \mathscr{L}\left(-D_{\kappa\left(x_{k}\right)^{-1} \chi^{-1}}\right), \quad s \mapsto x_{k} \cdot s,
$$

where $x_{k}$ acts via $R \rtimes G$-module structure of $\mathscr{F}$.

Denote by $B_{\chi, x_{k}}$ the effective Weil divisor on $Y$ where $\alpha_{\chi, x_{k}}$ vanishes. As shown in [9], Section $4.6, B_{\chi, x_{k}}$ is given by the formula

$$
B_{\chi, x_{k}}=D_{\chi^{-1}}+\left(x_{i}\right)-D_{\kappa\left(x_{k}\right)^{-1} \chi^{-1}} .
$$

Proposition 4.4. Each $B_{\chi, x_{k}}$ is supported on the union of the exceptional locus $\operatorname{Exc}(Y)$ of $Y \rightarrow \mathbb{C}^{3} / G$ and the strict transform of the surface $x_{k}^{|G|}=0$ in $\mathbb{C}^{3} / G$. 
Proof. Observe that it follows from the formula (4.5) that

$$
\sum_{\chi \in G^{\vee}} B_{\chi, x_{k}}=\sum_{\chi \in G^{\vee}} D_{\chi^{-1}}+|G|\left(x_{k}\right)-\sum_{\chi \in G^{\vee}} D_{\chi^{-1}}=|G|\left(x_{k}\right)=\left(x_{k}^{|G|}\right) .
$$

As each $B_{\chi, x_{k}}$ is effective, its support is contained in that of the principal Weil divisor $\left(x_{k}^{|G|}\right)$ on $Y$. This is precisely the strict transform of the surface $\left(x_{k}^{|G|}\right)$ in $\mathbb{C}^{3} / G$ union the exceptional locus.

The following simple result, which generalizes [2], Corollary 10.2, shows that $G$ being a subgroup of $\mathrm{SL}_{3}(\mathbb{C})$ together with $Y \rightarrow \mathbb{C}^{3} / G$ being crepant impose a strong restriction on the multiplicities of the exceptional divisors in $B_{\chi, i}$.

Proposition 4.5. Let $E$ be an irreducible exceptional divisor of $Y$ and $\mathrm{Q}(G)_{\mathscr{F}}$ a gnatfamily. In any triangle of $\mathrm{Q}(G)$, one of the three maps corresponding to $\mathrm{Q}(G)_{\mathscr{F}}$ vanishes along $E$ with multiplicity one while the other two do not vanish along $E$. Consequently, $E$ occurs in any $B_{\chi, i}$ with multiplicity zero or one.

Proof. Suppose the vertices of the triangle are $\chi, \kappa\left(x_{1}\right) \chi$ and $\kappa\left(x_{1} x_{2}\right) \chi$. Then by formula (4.6)

$$
B_{\chi, x_{1}}+B_{\kappa\left(x_{1}\right) \chi, x_{2}}+B_{\kappa\left(x_{1} x_{2}\right) \chi, x_{3}}=\left(x_{1} x_{2} x_{3}\right) .
$$

This is a $G$-invariant monomial since $G \subseteq \mathrm{SL}_{3}(\mathbb{C})$.

Since any $B_{\chi, x_{i}}$ is effective it suffices to show that $v_{E}\left(x_{1} x_{2} x_{3}\right)=1$ where $v_{E}$ is the valuation at $E$. This is in fact true for any crepant (and thus monomial) valuation of $K(Y)$ - see [7], in particular Step 5 of the proof of Theorem 1.4.

The relevance of the associated representation to the context of this paper is as follows. Let $\chi$ be a character of $G$. Recall the definition of the 3-dimensional cube quiver in Section 3. Define $\operatorname{Hex}(\chi)$ to be the subquiver of the McKay quiver which contains the six triangles which share the vertex $\chi$. This is depicted in Figure 4. The restriction of the representation $\mathrm{Q}(G)_{\mathscr{F}}$ to $\operatorname{Hex}(\chi)$ gives a representation $\operatorname{Hex}(\chi)_{\mathscr{F}}$. In the language of Section 3 this is a commutative cube of line bundles (which has been projected onto the plane). We can turn it into a skew-commutative cube as per (3.1). The following is then an immediate consequence of Proposition 2.3.

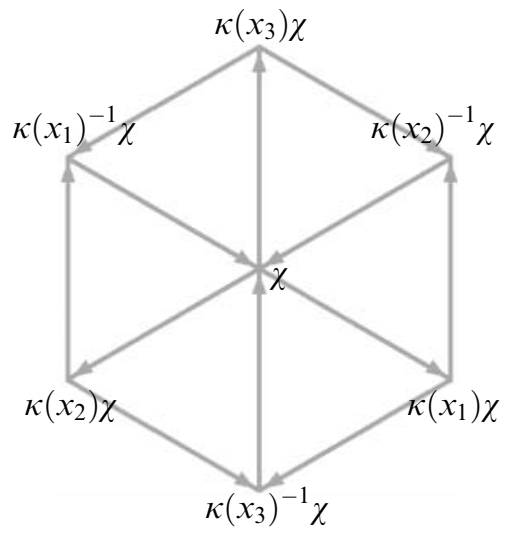

Figure 4. The subquiver $\operatorname{Hex}(\chi)$. 
Proposition 4.6. Let $\chi$ be a character of $G, Y \rightarrow \mathbb{C}^{3} / G$ a crepant resolution and $\mathscr{F}$ a gnat-family on $Y$. Then $\Psi_{\mathscr{F}}\left(\mathcal{O}_{0} \otimes \chi\right) \in D(Y)$ is the total complex of the skew-commutative cube of line bundles induced by $\operatorname{Hex}\left(\chi^{-1}\right)_{\mathscr{F}}$.

4.3. Behavior of $\mathbf{Q}(G)_{\mathscr{F}}$ along $\boldsymbol{E}$. Given a gnat-family $\mathscr{F}$ we would like to understand better the divisors $B_{\chi, x_{k}}$ where the maps $\alpha_{\chi, x_{k}}$ of $\mathrm{Q}(G)_{\mathscr{F}}$ vanish. In this section we fix one irreducible exceptional divisor $E$ and study the behavior of $\mathrm{Q}(G)_{\mathscr{F}}$ and its subrepresentations $\operatorname{Hex}(\chi)_{\mathscr{F}}$ generically along $E$.

Proposition 4.7. Fix a character $\chi \in G^{\vee}$. On the quiver $\operatorname{Hex}(\chi)$ we color an arrow black if the zero divisor of the corresponding map $\alpha$ in $\operatorname{Hex}(\chi)_{\mathscr{F}}$ contains $E$, otherwise we color it grey. Then all the possible colorings of $\operatorname{Hex}(\chi)$ are depicted in Figures 5-9.
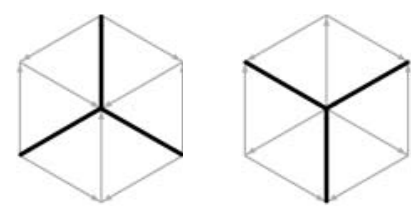

Figure 5. The (3,0)-sink and the $(0,3)$-sink.
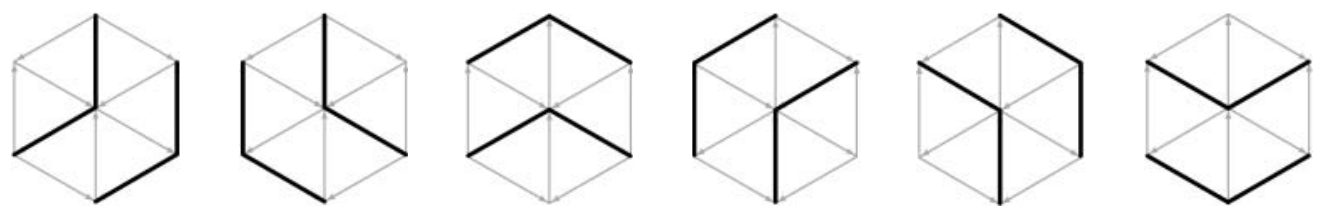

Figure 6. The $x_{1-}, x_{2}$ - and $x_{3}-(1,0)$-charges, the $x_{1-}, x_{2}$ - and $x_{3}-(0,1)$-charges.
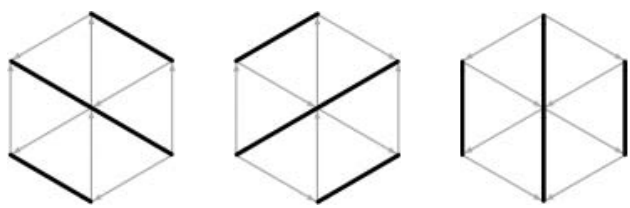

Figure 7. The $x_{1}$-tile, the $x_{2}$-tile and the $x_{3}$-tile.
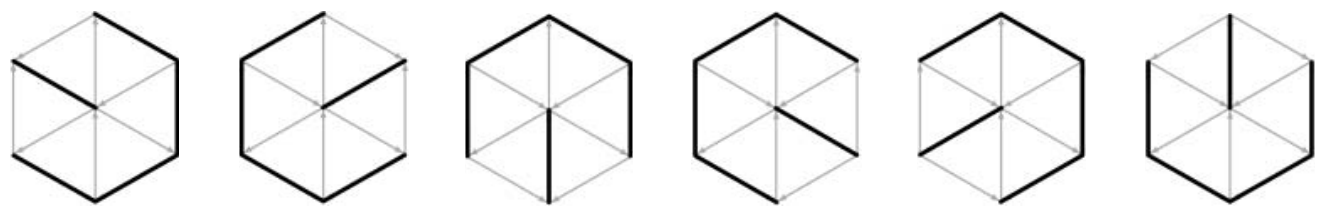

Figure $8 . \quad$ The $x_{1-}, x_{2}$ - and $x_{3}-(1,2)$-sources, the $x_{1-}, x_{2}$ - and $x_{3}-(2,1)$-sources.

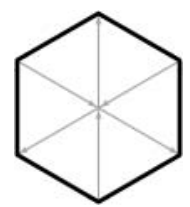

Figure 9. The (3, 3)-source. 
Proof. It follows from Proposition 4.5 that in each of the six triangles making up $\operatorname{Hex}(\chi)$ exactly one out of three arrows is colored black. It is a simple combinatorial exercise to check that all the possible configurations satisfying this condition are depicted in the Figures 5-9.

The terminology used in Figures 5-9 will be justified shortly as a consequence of the following combinatorial observations.

Corollary 4.8. Let $\chi$ be a character of $G$.

- If $\chi$ is an $x_{i}-(1,0)$-charge, then $\kappa\left(x_{i}\right) \chi$ is either another $x_{i}$-(1,0)-charge or a $(3,0)$ sink. While $\kappa\left(x_{i}\right)^{-1} \chi$ is either another $x_{i}-(1,0)$-charge, an $(3,3)$-source, an $x_{i}$-(1,2)-source or an $x_{j}-(2,1)$-source, where $j \neq i$.

- If $\chi$ is an $x_{i-}(0,1)$-charge, then $\kappa\left(x_{i}\right)^{-1} \chi$ is either another $x_{i}-(0,1)$-charge or a $(0,3)$ sink, while $\kappa\left(x_{i}\right) \chi$ is either another $x_{i}-(0,1)$-charge, an $(3,3)$-source, an $x_{i}-(2,1)$-source or an $x_{j}$-(1,2)-source, where $j \neq i$.

As $\kappa\left(x_{i}\right) \chi$ is the next vertex after $\chi$ in the direction of $x_{i}$-arrows and $\kappa\left(x_{i}\right)^{-1} \chi$ is the previous one, Corollary 4.8 may be interpreted as saying that charges propagate from a source to a sink in a straight line. The $x_{i}$ - $(1,0)$-charges propagate in the direction of $x_{i}$ arrows, while $x_{i}$ - $(0,1)$-charges propagate against the direction of $x_{i}$-arrows. We say that $x_{i}$ is the orientation of $x_{i}$-(1,0)-charge and that $(1,0)$ is its type (and the same for the other types of vertices).

Corollary 4.9. Let $\chi$ be a character of $G$.

- If $\chi$ is a (3,3)-source, then for $i \in\{1,2,3\}$ the vertex $\kappa\left(x_{i}\right) \chi$ is either an $x_{i}-(1,0)$ charge or a (3,0)-sink, while the vertex $\kappa\left(x_{i}\right)^{-1} \chi$ is either an $x_{i}-(0,1)$-charge or $a(0,1)$ sink.

- If $\chi$ is an $x_{i}$-(1,2)-source, then the vertex $\kappa\left(x_{i}\right) x_{i}$ is either an $x_{i}-(1,0)$-charge or $a$ $(3,0)$-sink, while each of the two vertices $\kappa\left(x_{j}\right) \chi$, for $j \neq i$, is either an $x_{j}$-(0,3)-charge or $a$ $(0,3)-\sin k$.

- If $\chi$ is an $x_{i}-(2,1)$-source, then the vertex $\kappa\left(x_{i}\right)^{-1} x_{i}$ is either an $x_{i}-(0,1)$-charge or a $(0,3)$-sink, while each of the two vertices $\kappa\left(x_{j}\right) \chi$, for $i \neq j$, is either an $x_{j}$-(3,0)-charge or $a$ $(3,0)-\operatorname{sink}$

Corollary 4.10. Let $\chi$ be a character of $G$.

- If $\chi$ is a $(3,0)$-sink, then each of the vertices $\kappa\left(x_{i}\right)^{-1} \chi$ for $i=1,2,3$ is either an $x_{i}-(1,0)$-charge, $(3,3)$-source, $x_{i}-(1,2)$-source or an $x_{j}-(2,1)$-source, where $j \neq i$.

- If $\chi$ is an $(0,3)$-sink, then each of the vertices $\kappa\left(x_{i}\right) \chi$ for $i=1,2,3$ is either an $x_{i}-(0,1)$-charge, $(3,3)$-source, $x_{i}-(2,1)$-source or an $x_{j}-(1,2)$-source, where $j \neq i$.

In other words, sources emit charges which propagate in a straight line to a sink. An $(a, b)$-source emits $a$ charges of type $(1,0)$ (which propagate in the direction of the arrows 
of the quiver) and $b$ charges of type $(0,1)$ (which propagate against the direction of the arrows). Similarly, an $(a, b)$-sink receives $a$ charges of type $(1,0)$ and $b$ charges of type $(0,1)$.

Corollaries 4.8-4.10 imply that given a gnat-family $\mathscr{F}$ and an exceptional divisor $E$ we can draw a graph on top of the McKay quiver $\mathrm{Q}(G)$ as follows. The vertices are the sources and sinks (with respect to the coloring of $\mathrm{Q}(G)$ keeping track of the vanishing of $E$ ) while the edges are the straight lines along which charges propagate from a source to a sink. We call it the sink-source graph and denote it $\mathrm{SS}_{\mathscr{F}, E}$.

The sink-source graph subdivides the torus $T_{H}$ into regions. The vertices interior to these regions are all tile vertices. Since two tiles can be adjacent only if they have the same orientation, we conclude that inside any one given region all the tiles have the same orientation $x_{i}$ for some $i \in 1,2,3$.

Example 4.11. Let $G$ be the group $\frac{1}{13}(1,5,7), Y$ be $G-\operatorname{Hilb}\left(\mathbb{C}^{3}\right)$ and let $\tilde{\mathscr{M}}$ be the dual of the universal family of $G$-clusters (cf. Section 6).

In Figure 10 the thick lines depict the sink-source graph $\mathrm{SS}_{\tilde{M}_{,} E_{7}}$ of the exceptional divisor $E_{7}$ (see Section 6.2) drawn on the torus $T_{H}$ (left). The lift of the graph to the universal cover $H \otimes \mathbb{R}^{2}$ is drawn on the right.
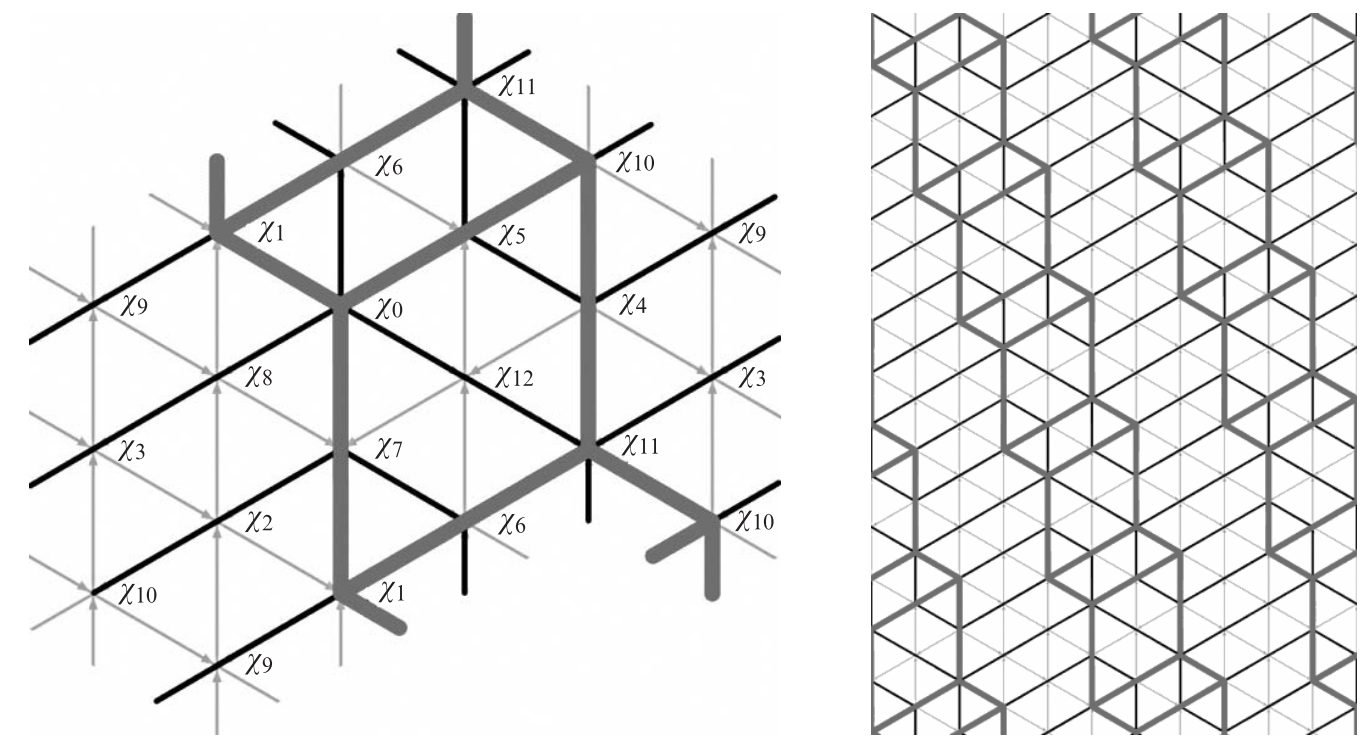

Figure 10. The sink-source graph of $E_{7}$ on $T_{H}$ (left) and on $H \otimes \mathbb{R}^{2}$ (right).

Looking at the left diagram, there are two sources (the $x_{2}-(1,2)$-source $\chi_{10}$ and the $x_{2}-(2,1)$-source $\left.\chi_{1}\right)$ and two sinks (the $(3,0)$-sink $\chi_{0}$ and the $(0,3)$-sink $\left.\chi_{11}\right)$.

4.4. Further properties of $\mathbf{Q}(G)_{\mathscr{F}}$. We now restrict our attention more to the case where $Y=G$-Hilb $\left(\mathbb{C}^{3}\right)$ and $\mathscr{F}=\tilde{\mathscr{M}}$ is the dual of the universal family of $G$-clusters. 
Given a single $G$-cluster $\mathscr{V}$ its associated representation $\mathrm{Q}(G)_{\mathscr{V}}$ is the vector space underlying the corresponding $R \rtimes G$-module $V=\Gamma(\mathscr{V})$. The grading $\bigoplus_{\chi} V_{\chi}$ is given by the natural decomposition of $V$ into irreducible $G$-representations. The map corresponding to an arrow $\left(\chi, x_{k}\right)$ is given by the multiplication map $V_{\chi} \stackrel{\cdot x_{k}}{\rightarrow} V_{\chi \kappa}\left(x_{k}\right)$.

We begin with a simple observation about $G$-clusters.

Proposition 4.12. If $\mathscr{V}$ is a single G-cluster then in the associated representation of $\mathscr{V}$ one of the arrows leading into the vertex $\chi \neq \chi_{0}$ must be non-zero. If $\mathscr{V}$ is the dual of a single $G$-cluster then one of the arrows leading out of $\chi$ must be non-zero.

Proof. Since a $G$-cluster is of the form $\mathcal{O}_{Z}$ for a subscheme $Z \subset \mathbb{C}^{3}$, the $R \rtimes G$ module $V$ corresponding to $\mathscr{V}$ is of the form $R / I$ for some $G$-invariant ideal $I$. Therefore it is generated by 1 as an $R$-module. In particular, for any $\chi \neq \chi_{0}$ there exists a monomial $1 \neq m \in R$ such that $m \cdot 1$ is a non-zero element of $V_{\chi}$. Writing $m=x_{i} m^{\prime}$ for some $x_{i}$ we find that the arrow labeled $x_{i}$ leading into $\chi$ must be a non-zero map.

The dual claim follows since taking duals corresponds to reversing all the arrows (and relabeling $\chi$ by $\chi^{-1}$ ).

Corollary 4.13. Let $Y=G-\operatorname{Hilb}\left(\mathbb{C}^{3}\right)$ and $\tilde{\mathscr{M}}$ the dual of the universal family of $G$ clusters. If $\chi \in G^{\vee}$ is non-trivial then the intersection of the three divisors $B_{\chi, 1}, B_{\chi, 2}$ and $B_{\chi, 3}$ associated to $\mathrm{Q}(G) \tilde{\mathscr{M}}$ is empty.

Proof. Over any point belonging to the intersection of $B_{\chi, 1}, B_{\chi, 2}$ and $B_{\chi, 3}$ we get the dual of a $G$-cluster for which all the maps leading out of $\chi$ are zero (remember that $B_{\chi, i}$ records the locus where the map $\alpha_{\chi, i}$ is zero). This cannot happen by Proposition 4.12 so the intersection must be empty.

Proposition 4.14. Let $Y$ be $G-\operatorname{Hilb}\left(\mathbb{C}^{3}\right), E \in \operatorname{Exc}(Y)$ an irreducible exceptional divisor and $\mathscr{M}$ the universal family of $G$-clusters. Then the sink-source graph $\mathrm{SS}_{\mathscr{M}, E}$ contains exactly one $(0,3)$-sink (given by the vertex $\left.\chi_{0}\right)$. Similarly, the sink-source graph $\mathrm{SS}_{\tilde{M}, E}$ contains exactly one $(3,0)$-sink (given by the vertex $\chi_{0}$ ).

Proof. It suffices to prove the first claim as the dual claim follows similarly. If $\chi$ is a $(0,3)$-sink in $\mathrm{SS}_{\mathscr{M}, E}$ then in the associated representation of a fiber of $\mathscr{M}$ over any point in $E$ all three arrows leading into $\chi$ are zero-maps. This, by Proposition 4.12 , can only happen if $\chi=\chi_{0}$.

It remains to show that $\chi_{0}$ is a $(0,3)$-sink in $\mathrm{SS}_{\mathscr{M}, E}$. If not then there exists a point $y \in E$ such that in the associated representation of $\left.\mathscr{M}\right|_{y}$ one of the three arrows leading into $\chi_{0}$ is not a zero map. Since the same is also true for every $\chi \neq \chi_{0}$ (by Proposition 4.12), we can find a path of non-zero maps in the McKay quiver which starts and ends at the same vertex. Since each of these maps is multiplication by non-zero number their composition is also non-zero. So this path gives a monomial $1 \neq m \in R$ which is invariant under $G$ (since the path starts and ends at the same vertex) such that $m \cdot 1 \neq 0$ in $\left.\mathscr{M}\right|_{y}$. 
This is impossible since the resolution $Y \stackrel{\pi}{\rightarrow} \mathbb{C}^{3} / G$ is the Hilbert-Chow map of $\mathscr{M}$ and so for any monomial $1 \neq m \in R^{G}$ and $\left.s \in \mathscr{M}\right|_{y}$ we have $m \cdot s=m(\pi(y)) s=m(0) s=0$. Here $\pi(y)=0$ since $y$ lies in the exceptional locus of $Y$.

The next result is a consequence of 4.14. It will be heavily used to derive properties of the associated representation $\mathrm{Q}(G)_{\tilde{\mathscr{M}}}$.

Proposition 4.15. For any $Y, \mathscr{F}$ and $E$, if the sink-source graph $\mathrm{SS}_{\mathscr{F}, E}$ contains exactly one $(3,0)$-sink then there are exactly three possibilities for its remaining vertices:

(1) One (0,3)-sink and one (3,3)-source.

(2) One (0,3)-sink, one (1,2)- and one (2,1)-source.

(3) Two (0,3)-sinks and three (1,2)-sources.

Proof. If there exists only one $(3,0)$-sink, all the sources together must emit exactly three charges of type $(1,0)$. This leaves us with three possibilities for the sources: either one $(3,3)$-source, or one $(1,2)$ - and one $(2,1)$-source, or three $(1,2)$-sources. The statement about sinks follows by counting in each case the number of $(0,1)$-charges emitted.

Corollary 4.16. If the sink-source graph $\mathrm{SS}_{\mathscr{F}, E}$ contains exactly one $(3,0)$-sink then on the torus $T_{H}$ it looks like either Figure 11(a), Figure 11(b) or Figure 11(c) (up to the lengths of the sides and in the case of Figure 11(a) up to a rotation by $\pm 2 \pi / 3$ ).

Proof. On the universal cover $H \otimes \mathbb{R}^{2}$ take any vertex projecting to the $(3,0)$-sink $O_{1}$ in $T_{H}$ and denote it by $A$. Follow the three $(1,0)$-charge lines which enter $A$ back to their respective sources. Denote the source emitting the $x_{i}-(1,0)$-charge line by $B_{i}$. We obtain, up to the actual lengths of $A B_{i}$, the picture in Figure 12.

By Proposition 4.15, $B_{1}, B_{2}$ and $B_{3}$ project in $T_{H}$ either to the same $(3,3)$-source or to a $(1,2)$-source and a $(2,1)$-source or to three distinct $(1,2)$-sources. We treat here the case of a $(1,2)$-source and a $(2,1)$-source, which leads to Figure 11(a) and its rotations. The other two cases are analogous and lead to configurations in Figures 11(b) and 11(c).

By our assumption exactly two of $B_{i}$ must project to same vertex in $T_{H}$. Assume they are $B_{1}$ and $B_{3}$-two other possibilities lead to rotations of the resulting configuration. Then the image of $B_{1}$ and $B_{3}$ in $T_{H}$ emits an $x_{1}-(1,0)$-charge and an $x_{2}-(1,0)$-charge. This means it must be an $x_{3}-(2,1)$-source (check with the list of source vertices in Figure 8). Since we have one $(3,0)$-sink and one $(0,3)$-sink, the two sources must emit together one $x_{i}$-(1,0)and one $x_{i}-(0,1)$-charge for $i \in 1,2,3$. The source $B_{2}$ projects to has therefore to be an $x_{3}-(1,2)$-source.

Consider now the $x_{1}-(0,1)$-charge line emitted by $B_{2}$ and the $x_{3}-(0,1)$-charge line emitted by $B_{1}$. We claim that the vertex $B_{12}$ at the intersection of these lines projects to the $(0,3)$-sink in $T_{H}$. If not then one of the two charge lines would have to terminate in the $(0,3)$-sink before intersecting the other. Then, from Figure 12, one of the other charge lines ending in this sink would have to come from within the parallelogram $A B_{1} B_{2} B_{12}$. As this charge line can't cross $A B_{1}$ or $A B_{2}$, its source must also lie within this parallelogram. 


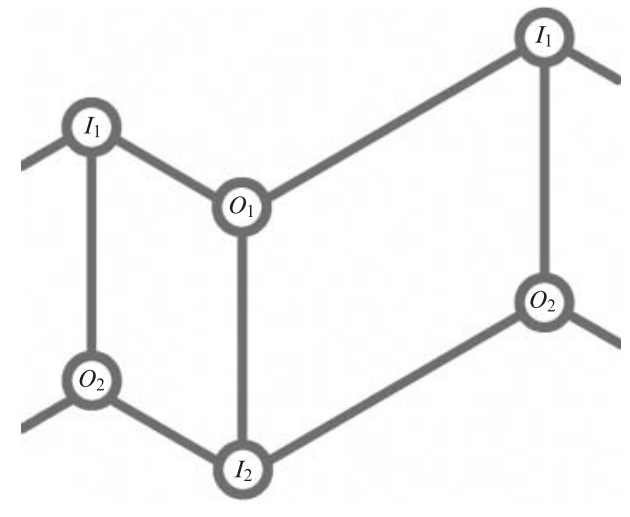

(a) One $(1,2)$-source and one $(2,1)$-source.

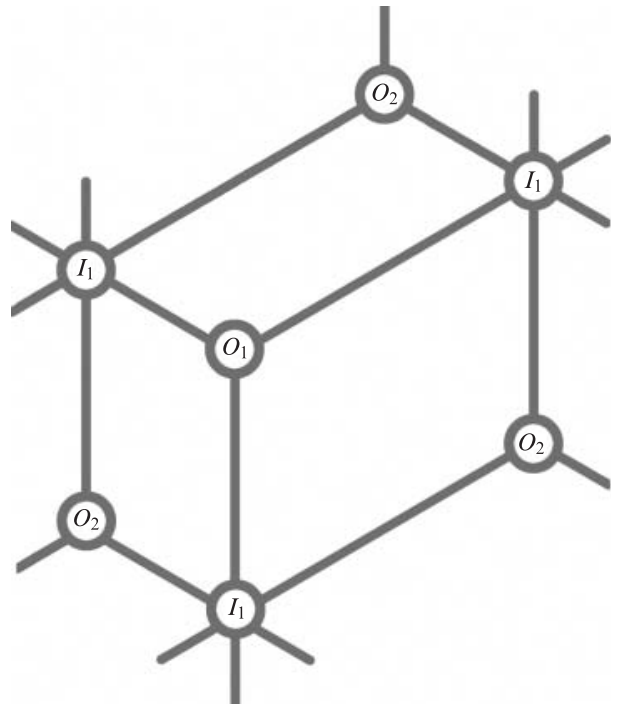

(b) A single (3,3)-source.

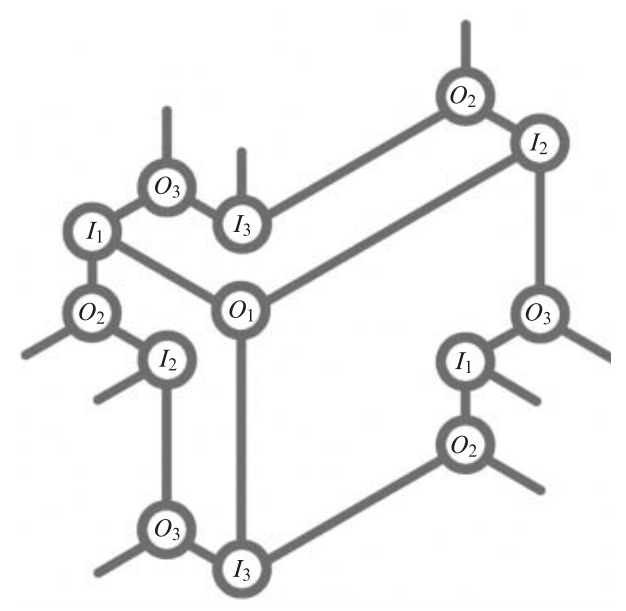

(c) Three $(1,2)$-sources.

Figure 11. Sink-source graphs in the case of a single $(3,0)$-sink.

This is impossible - every vertex in edges $A B_{1}$ and $A B_{2}$ projects to a distinct vertex in $T_{H}$, and therefore every vertex in the parallelogram $A B_{1} B_{2} B_{12}$ must also project to a distinct vertex. But by assumption there are only two source vertices in $T_{H}$ and $B_{1}$ and $B_{2}$ already project to those. Therefore no other vertex within $A B_{1} B_{2} B_{12}$ can project to a source vertex.

Thus $B_{12}$ projects to the $(0,3)$-sink and drawing in all the remaining charge lines yields the configuration on Figure 11(a).

We now present a few more properties of the associated representation of $\tilde{\mathscr{M}}$-the dual of the universal family of $G$-clusters. 


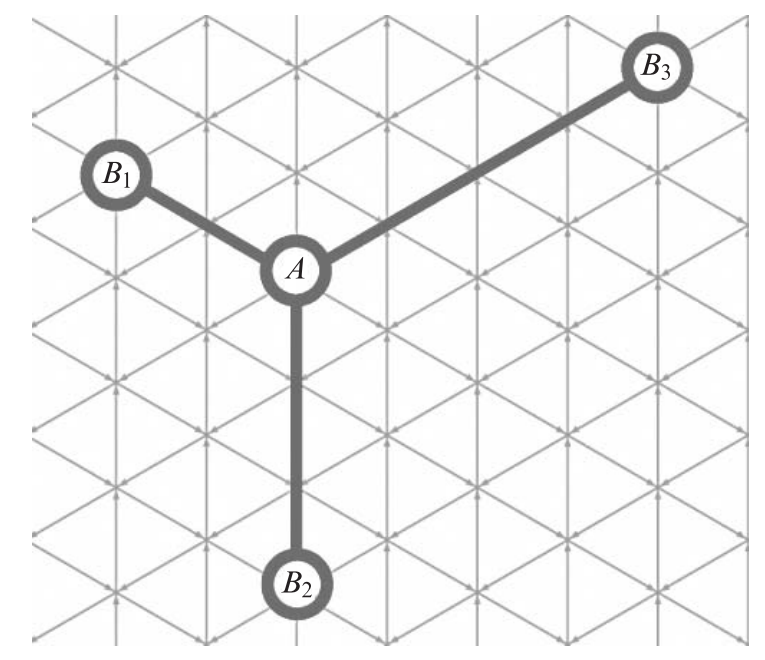

Figure 12. The lift of $(3,0)$-sink and its three charge lines to $H \otimes \mathbb{R}^{2}$.

Proposition 4.17. Let $Y$ be $G-\operatorname{Hilb}\left(\mathbb{C}^{3}\right)$ and $\tilde{\mathscr{M}}$ be the dual of the universal family of $G$-clusters. Let $E$ and $F$ be two irreducible exceptional divisors on $Y$. Suppose $\chi \in G^{\vee}$ such that in $\mathrm{Q}(G)_{\tilde{M}}$ we have $E \subseteq B_{\chi, i}, B_{\chi, j}$, while $F \subseteq B_{\chi \kappa}\left(x_{i}\right), j, B_{\chi \kappa}\left(x_{j}\right), i$ for some $i \neq j$, cf. Figure 13. Then $E$ and $F$ do not intersect.

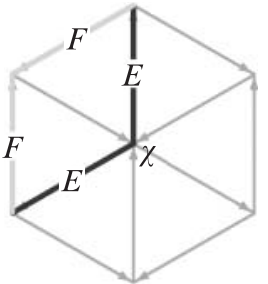

(a)

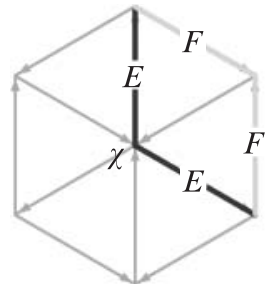

(b)

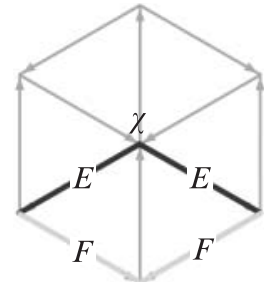

(c)

Figure 13. The configurations from Proposition 4.17.

Proof. We give the proof under the assumption that the sink-source graph $\mathrm{SS}_{\tilde{\mathscr{M}}, F}$ is the one depicted in Figure 11(a). The cases of $\mathrm{SS}_{\tilde{\mathscr{M}}, F}$ being as in Figures 11(b) and 11(c) are similar and simpler, each requiring only a part of the argument presented below.

We use the notation in Figure 11(a) to label the vertices of $\mathrm{SS}_{\tilde{M}_{, F}}: O_{1}$ is the $(3,0)$ sink, $O_{2}$ the $(0,3)$-sink, $I_{1}$ the $x_{3}-(2,1)$-source and $I_{2}$ the $x_{3}-(1,2)$-source. By Proposition 4.14, $O_{1}=\chi_{0}$ is also the unique $(3,0)$-sink in $\mathrm{SS}_{\tilde{\mathscr{M}}, E}$.

We first deal with the configuration in Figure 13(c). The proof will require working with both sink-source graphs $\mathrm{SS}_{\tilde{\mathscr{M}}, E}$ and $\mathrm{SS}_{\tilde{\mathscr{M}}, F}$ which we superimpose on the torus $T_{H}$.

First note that the vertex $\chi$ must be an $x_{3}-(1,0)$-charge for $E$. All such charges lie on the $x_{3}-(1,0)$-charge line in $\mathrm{SS}_{\tilde{\mathscr{M}}, E}$ which terminates at $O_{1}$. We claim that the source in $\mathrm{SS}_{\tilde{\mathscr{M}}, F}$ for this charge line must lie below the vertex $\kappa\left(x_{3}\right)^{-1} I_{2}$-see Figure 11(a). If this were not the case then every vertex which is an $x_{3}-(1,0)$-charge for $E$ would be either an 
$x_{3}-(1,0)$ charge or the source $I_{2}$ for $F$. This would render Figure 13(c) impossible. Hence $\kappa\left(x_{3}\right)^{-1} I_{2}$ is an $x_{3}-(1,0)$-charge for $E$ and we have the configuration depicted in Figure 14. Since all three arrows leading out of $\kappa\left(x_{3}\right)^{-1} I_{2}$ are marked by either $E$ or $F$ we must have $E \cap F=\emptyset$ by Corollary 4.13 .

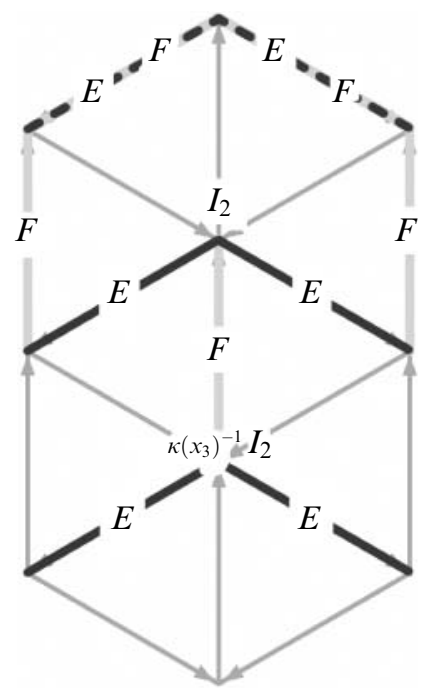

Figure 14. Partial divisor configuration for $E$ and $F$ near $\kappa\left(x_{3}\right)^{-1} I_{2}$.

Next we assume the configuration in Figure 13(b). This also deals with the configuration in Figure 13(a) as the argument there is identical.

As we assumed that $\mathrm{SS}_{\tilde{\mathscr{M}}, F}$ looks like in Figure 11(a), for $F$ vertex $\chi$ can only be an $x_{2}-(0,1)$-charge or the $x_{3}-(1,2)$-source $O_{2}$. So $\chi$ must lie on the $x_{2}-(0,1)$-charge line $I_{2} O_{2}$ in $\mathrm{SS}_{\tilde{\mathscr{M}}, F}$. On the other hand $\chi$ must lie on the $x_{2}-(1,0)$-charge in $\mathrm{SS}_{\tilde{\mathscr{M}}, E}$ which terminates at $O_{1}$ (remember that $O_{1}$ is also the $(3,0)$-sink for $E$ ). Looking at Figure 11(a) one sees that on the torus $T_{H}$ the path along this charge line from $\chi$ to $O_{1}$ must pass through both $I_{2}$ and $I_{1}$. Since charge lines for $E$ can't cross each other, the source in $\mathrm{SS}_{\tilde{\mathcal{M}}, E}$ for the $x_{1}-(1,0)$-charge (resp. the $x_{3}-(1,0)$-charge) line must lie strictly between $O_{1}$ and $I_{1}$ (resp. between $O_{1}$ and $I_{2}$ ). Denoting this source by $P_{1}\left(\right.$ resp. $P_{2}$ ) we obtain the configuration in Figure 15.

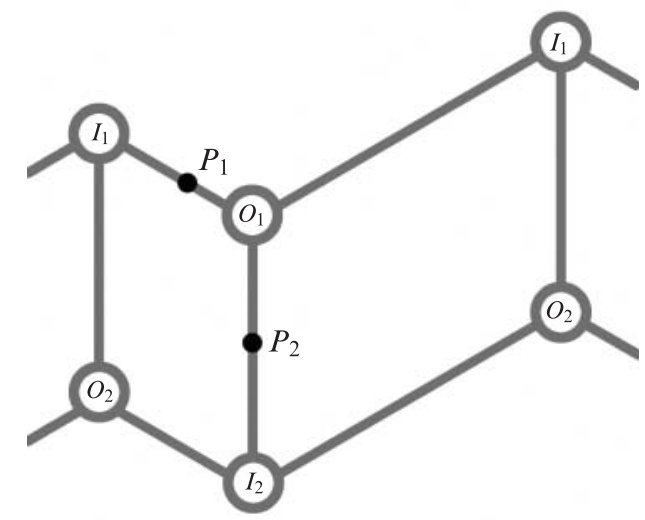

Figure 15. The sink-source graph $\mathrm{SS}_{\tilde{M}, F}$ with the sources $P_{1}$ and $P_{2}$ for $E$. 
Suppose $\mathrm{SS}_{\tilde{\mathscr{M}}, E}$ is shaped as in Figures 11(a) or 11(b). Then it has at most two sources. Therefore the $x_{2}-(1,0)$-charge line in $\mathrm{SS}_{\tilde{M}, E}$ must originate at either $P_{1}$ or $P_{2}$. Suppose it originates at $P_{1}$, the other case is analogous. Then $P_{1}$ is an $x_{3}-(2,1)$-source for $E$ since this is the unique source which emits both $x_{2}-(1,0)$ - and $x_{1}-(1,0)$-charges. We claim that $\kappa\left(x_{2}\right) P_{1}$ lies within the left parallelogram $O_{1} I_{2} O_{2} I_{1}$ in Figure 15 . Then $\kappa\left(x_{2}\right) P_{1}$ has to be an $x_{2}$ tile for $F$ which gives the configuration in Figure 16, whence $E \cap F=\emptyset$ by Corollary 4.13. Observe, as $I_{1}$ is an $x_{2}-(1,0)$-charge for $E, P_{1}$ can not be the vertice $\kappa\left(x_{1}\right) I_{1}$, by direct inspection. Also, the side $O_{1} P_{2} I_{2}$ is at least two edges long as it has to accommodate three vertices. These two observations show that, as claimed, $\kappa\left(x_{2}\right) P_{1}$ must lie in the interior of $O_{1} I_{2} O_{2} I_{1}$.

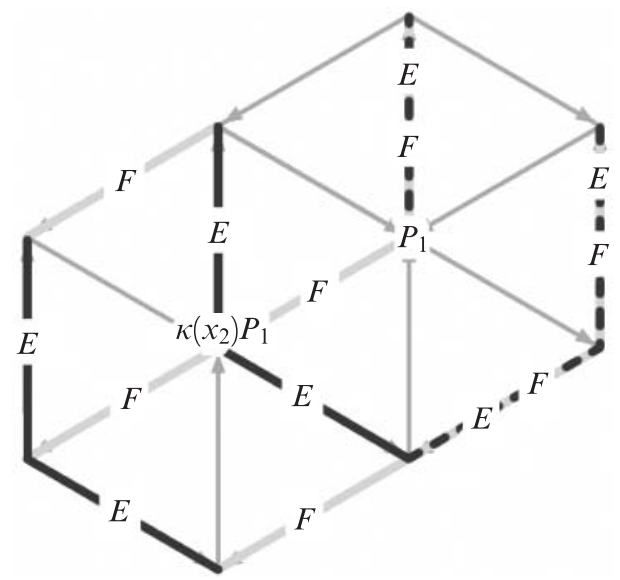

Figure 16. Divisor configuration for $E$ and $F$ near $\kappa\left(x_{2}\right) P_{1}$.

Finally, suppose $\mathrm{SS}_{\tilde{\mathscr{M}}, E}$ is shaped as in Figure 11(c). Then $x_{2}-(1,0)$-charge line in $\mathrm{SS}_{\tilde{\mathscr{M}}, E}$ originates at an $x_{2}-(1,2)$-source distinct from $P_{1}$ and $P_{2}$. Denote it by $P_{3}$. But then looking at Figure 11(c) (and keeping in mind that there $P_{1}$ is $O_{1}, P_{2}$ is $O_{3}$ and $P_{3}$ is $O_{2}$ ) we see that the vertex $\kappa\left(x_{2}\right) P_{3}$ must lie within the parallelogram formed by $O_{1} P_{1}$ and $O_{1} P_{2}$. Therefore, from Figure 15 it certainly lies within the left parallelogram $O_{1} I_{2} O_{2} I_{1}$. We conclude that $\kappa\left(x_{2}\right) P_{3}$ is an $x_{2}$-tile in $\mathrm{SS}_{\tilde{\mathscr{M}}, F}$. This gives a configuration similar to that in Figure 16, showing again that $E \cap F=\emptyset$ by Corollary 4.13 .

Corollary 4.18. Let $Y$ be $G-\operatorname{Hilb}\left(\mathbb{C}^{3}\right)$ and $\tilde{\mathscr{M}}$ be the dual of the universal family of $G$-clusters. Let $E$ and $F$ be two irreducible exceptional divisors on $Y$. Suppose $\chi \in G^{\vee}$

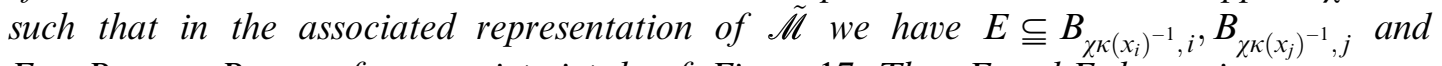
$F \subseteq B_{\chi \kappa\left(x_{k}\right), i}, B_{\chi \kappa}\left(x_{k}\right), j$ for some $i \neq j \neq k, c f$. Figure 17. Then $E$ and $F$ do not intersect.

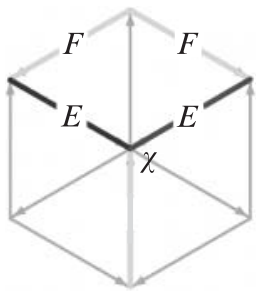

(a)

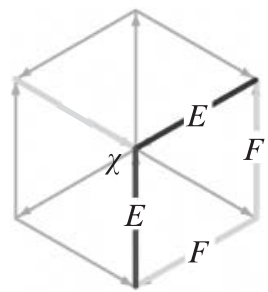

(b)

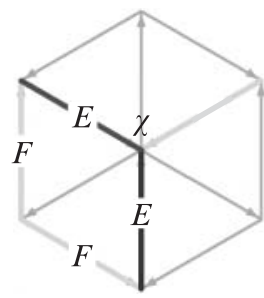

(c)

Figure 17. The configurations from Corollary 4.18. 
Proof. If in the configuration in Figure 17(a) we re-center on the vertex $\kappa\left(x_{3}\right) \chi$, we obtain in $\operatorname{Hex}\left(\kappa\left(x_{3}\right) \chi\right)$ the configuration in Figure 13(a). By Proposition 4.17 we obtain $E \cap F=\emptyset$. The other two cases follow similarly.

Proposition 4.19. Let $Y$ be $G-\operatorname{Hilb}\left(\mathbb{C}^{3}\right)$ and $\tilde{\mathscr{M}}$ be the dual of the universal family of $G$-clusters. Let $D, E$ and $F$ be irreducible exceptional divisors on $Y$. Suppose $\chi \in G^{\vee}$ such

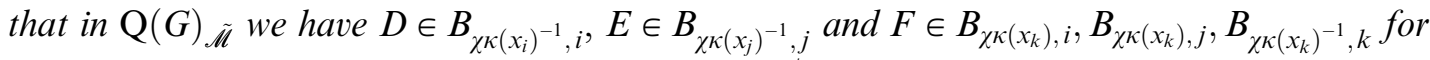
some $i \neq j \neq k$, cf. Figure 18. Then $D \cap E \cap F=\emptyset$.

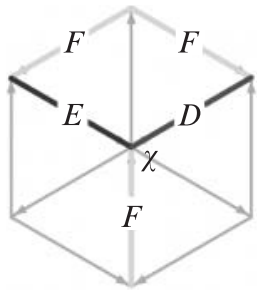

(a)

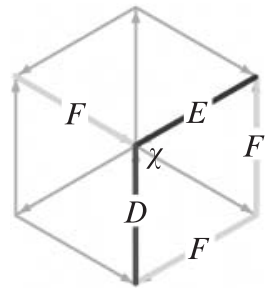

(b)

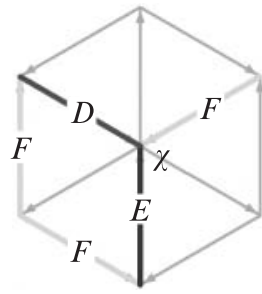

(c)

Figure 18. The configurations from Proposition 4.19.

Proof. We give the proof for the configuration in Figure 18(a), the other two cases are proven similarly.

Comparing Figure 18(a) to Figures 5-9 we find that in $\mathrm{SS}_{\tilde{\mathscr{M}}, F}$ the vertex $\chi$ can only be the $x_{3}-(1,2)$-source. Therefore $\mathrm{SS}_{\tilde{\mathscr{M}}, F}$ is as depicted in Figure 11(a) (no rotation necessary) or in Figure 11(c). Assume the former. Using the same notation as in Figure 11(a), note that $\chi=I_{2}$.

Denote by $P_{1}$ the source of $x_{3}-(1,0)$-charge line for $E$. Since this charge line also terminates at $O_{1}$ the source $P_{1}$ must lie below $O_{1}$. If it lies below $I_{2}$ then the configuration in Figure 14 would occur, implying $E \cap F=\emptyset$ (by Corollary 4.13). $P_{1}$ cannot equal $I_{2}$ since then $\operatorname{Hex}\left(I_{2}\right)$ would not look as in Figure 18(a). So we can assume $P_{1}$ lies strictly between $O_{1}$ and $I_{2}$.

Suppose now that $P_{1} \neq \kappa\left(x_{3}\right) I_{2}$. Then $\kappa\left(x_{3}\right)^{-1} P_{1}$ is an $x_{3}-(1,0)$-charge in $\mathrm{SS}_{\tilde{M}_{, F}}$. Also, $P_{1}$ must be either an $x_{3}-(1,2)$-source, an $x_{1}-(2,1)$-source or an $x_{2}-(2,1)$-source in $\mathrm{SS}_{\tilde{\mathscr{M}}, E}$. If it is an $x_{3}-(1,2)$-source we get the configuration depicted in Figure 19, which by Corollary 4.13 means $E \cap F=\emptyset$. If $P_{1}$ is an $x_{2}-(2,1)$-source, then we claim that $\kappa\left(x_{1}\right) P_{1}$ is an interior vertex of the right parallelogram in Figure 11(a) and so we have the configuration in Figure 20, implying $E \cap F=\emptyset$. The argument for $P_{1}$ being an $x_{1}-(2,1)$ source is the same but with the vertex $\kappa\left(x_{2}\right) P_{1}$.

It suffices to show that the side $O_{1} I_{1}$ is more than one edge long, as then $\kappa\left(x_{1}\right) P_{1}$ can't lie on the side $I_{1} O_{2}$ and has to be an interior vertex. Observe that the $x_{1}-(1,0)$-charge line for $E$ begins at $P_{1}$ and terminates at $O_{1}$, and so it must pass through $I_{1}$ (see Figure 11(a)). So $I_{1}$ is an $x_{2}-(1,0)$-charge for $E$. Therefore the side $O_{1} I_{1}$ is more than one edge long-a $(3,0)$-source $O_{1}$ and an $x_{2}$-(1,0)-charge $I_{1}$ could not be adjacent in such a way. 


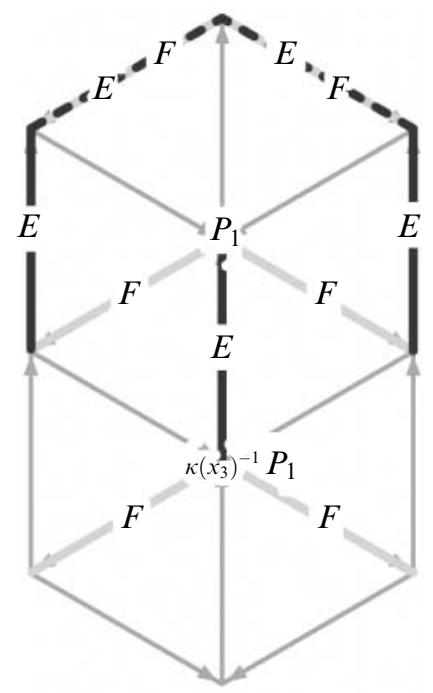

Figure 19. Partial divisor configuration for $E$ and $F$ near $P_{1}$.

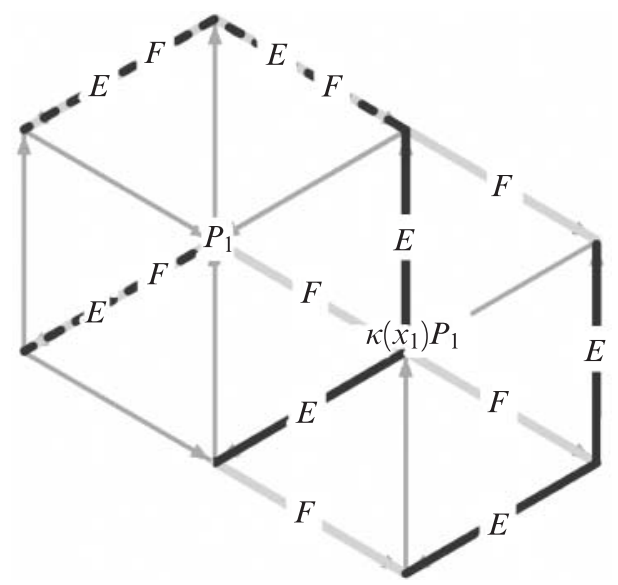

Figure 20. Divisor configuration for $E$ and $F$ near $\kappa\left(x_{1}\right) P_{1}$.

Finally, suppose $P_{1}=\kappa\left(x_{3}\right) I_{2}$. Recall that in $\mathrm{SS}_{\tilde{M}_{E}}$ the vertex $P_{1}$ is either an $x_{3}-(1,2)$-source, an $x_{1}-(2,1)$-source or an $x_{2}-(2,1)$-source. The first and the third options are incompatible with the data of Figure 18 (a) since $\chi=I_{2}$. So $P_{1}$ is an $x_{1}-(2,1)$-source for $E$. Now the $x_{2}-(1,0)$-charge line for $E$ begins at $P_{1}$ and terminates at $O_{1}$ so, looking at Figure 11(a), it must pass through $I_{1}$. We conclude that $I_{1}$ is an $x_{2}-(1,0)$-charge for $E$.

Repeating the entire argument from the start with $D$ instead of $E$ yields that either $D \cap F=\emptyset$ or $I_{1}$ is an $x_{1}-(1,0)$-charge for $D$. So $I_{1}$ is an $x_{1}-(1,0)$-charge for $D$, an $x_{2}-(1,0)$-charge for $E$ and the $x_{3}-(1,2)$-source for $F$ (its original definition). This gives the configuration in Figure 21, implying $D \cap E=\emptyset$ by Corollary 4.13 .

The argument for $\mathrm{SS}_{\tilde{\mathscr{M}}, F}$ being as in Figure 11(c) is very similar, so we only give a brief outline. We again denote by $P_{1}$ the source of $x_{3}-(1,0)$-charge line for $E$. But now the 


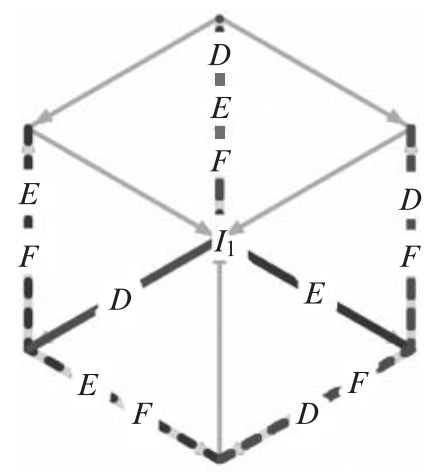

Figure 21. Divisor configuration for $D, E$, and $F$ near $I_{1}$.

case $P_{1}=\kappa\left(x_{3}\right) I_{3}$ leads immediately to a contradicting configuration at $\kappa\left(x_{2}\right)^{-1} I_{2}$. The difficulty is when $P_{1}$ is an $x_{1}$-(2,1)-source lying somewhere higher between $O_{1}$ and $I_{3}$ while $\kappa\left(x_{2}\right) P_{1}$ is $I_{2}$. It turns out in such case the $x_{1}-(1,2)$-source for $E$ has to be $\kappa\left(x_{1}\right) I_{1}$ (otherwise $\chi=I_{3}$ would be an $x_{3}$-tile for $E$ contradicting Figure 18(a)). Denoting by $P_{2}$ the source of $x_{3}$-(1,0)-charge line for $D$ and repeating the same argument as for $E$, we now have deal with the case where $P_{2}$ is an $x_{2}-(2,1)$-source lying somewhere on $O_{1} I_{3}$ and $\kappa\left(x_{1}\right) P_{2}=I_{1}$. But then $P_{2}=\kappa\left(x_{1}\right)^{-1} I_{1}$ is an $x_{2}-(2,1)$-source for $D$ and $\kappa\left(x_{1}\right) I_{1}$ is a $x_{1}$-(1,2)-source for $E$, which creates a contradicting configuration at the vertex $I_{1}$ lying between them.

Proposition 4.20. Let $Y$ be $G$-Hilb $\left(\mathbb{C}^{3}\right)$ and $\tilde{\mathscr{M}}$ be the dual of the universal family of $G$-clusters. Let $E$ and $F$ be irreducible exceptional divisors on $Y$. Suppose there exists $\chi \in G^{\vee}$ such that for $\tilde{\mathscr{M}}$ we have $E \in B_{\chi \kappa\left(x_{i}\right)^{-1}, i}, B_{\chi \kappa\left(x_{j}\right)^{-1}, j}$ and $F \in B_{\chi \kappa\left(x_{i}\right), j}, B_{\chi \kappa\left(x_{i}\right), k}, B_{\chi \kappa}\left(x_{j}\right), i, B_{\chi \kappa}\left(x_{j}\right), k$ for some $i \neq j \neq k$, cf. Figure 22. Then $E \cap F=\emptyset$.

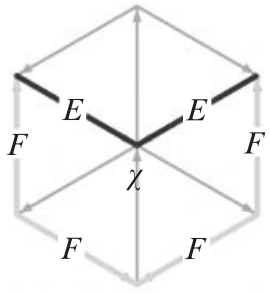

(a)

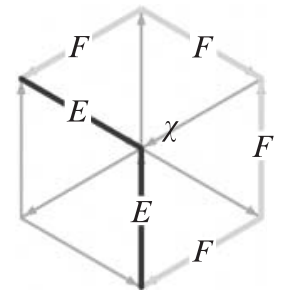

(b)

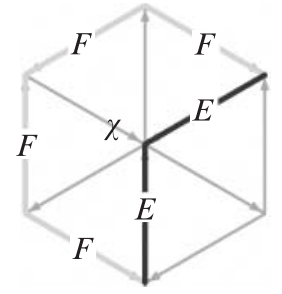

(c)

Figure 22. The configurations from Proposition 4.20.

Proof. We give the proof for the configuration in Figure 22(a). The other two are identical.

Comparing Figure 22(a) with Figures 5-9 we conclude that in $\mathrm{SS}_{\tilde{\mathscr{M}}, F}$ the vertex $\chi$ must be a $(3,3)$-source or an $x_{3}-(2,1)$-source.

Suppose the latter. Then $\mathrm{SS}_{\tilde{M}_{, F}}$ is as depicted in Figure 11(a) (no rotation necessary). Observe that $\chi=I_{1}$. Let $P_{1}$ and $P_{2}$ denote the sources of the $x_{1}$ - and $x_{2}$-(1,0)-charge lines for $E$, respectively. From Figure $22(\mathrm{a})$ we see that $I_{1}$ can neither be an $x_{1}-(1,0)$-charge 
nor $x_{2}-(1,0)$-charge for $E$. Therefore $P_{1}$ (resp. $\left.P_{2}\right)$ lies on the $x_{1}-(1,0)$-charge line (resp. the $x_{2}-(1,0)$-charge line) as depicted in Figure 23.

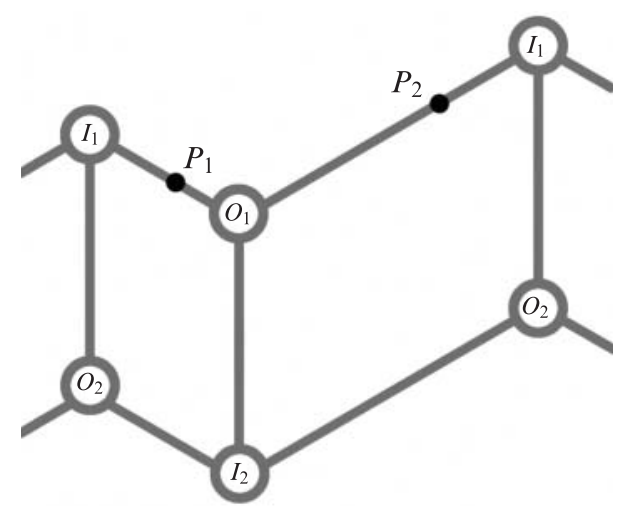

Figure 23. The sink-source graph $\mathrm{SS}_{\tilde{\mathscr{M}}, F}$ with sources $P_{1}$ and $P_{2}$ for $E$.

Since $P_{1} \neq P_{2}$ the graph $\mathrm{SS}_{\tilde{\mathscr{M}}, E}$ is as in 11 (a) (up to rotation) or as in 11(c). In former case we find that either $P_{1}$ is an $x_{1}-(1,2)$-source or $P_{2}$ is an $x_{2}-(1,2)$-source and in latter case we find that both of these are true. Suppose $P_{1}$ is an $x_{1}-(1,2)$-source, the other case is similar. Since $I_{1}=\chi$ the vertex $I_{1} \kappa\left(x_{1}\right)$ can't be an $x_{1}-(1,2)$-source for $E$ by inspection of Figure 18(a). So $P_{1} \neq I_{1} \kappa\left(x_{1}\right)$ and hence $\kappa\left(x_{1}\right)^{-1} P_{1}$ is an $x_{1}-(1,0)$-charge for $F$ and get the configuration depicted in Figure 24. As usual, it implies $E \cap F=\emptyset$ by Corollary 4.13.

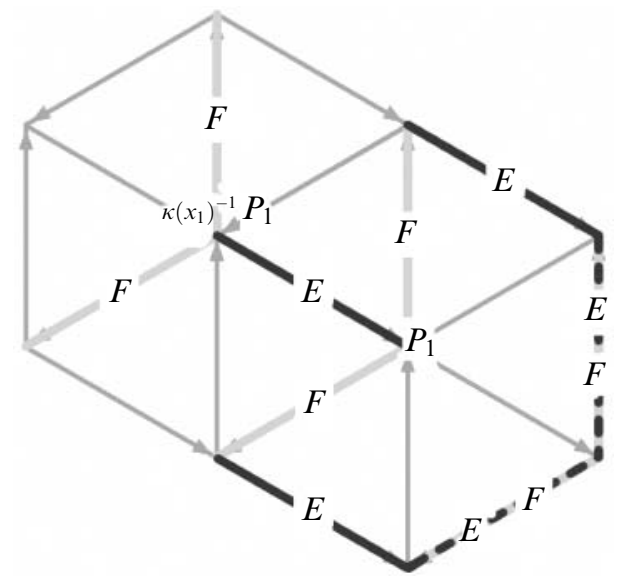

Figure 24. Divisor configuration for $E$ and $F$ near $P_{1}$.

It remains to consider the case when $\chi$ is a $(3,3)$-source for $F$. This time the sinksource graph $\mathrm{SS}_{\tilde{M}, \mathscr{F}}$ is as in Figure $11(\mathrm{~b})$. Reasoning as above, the vertices $P_{1}, P_{2}$ and $P_{3}$ are configured as in Figure 25. However, observe that in both Figures 11(b) and 11(c) the three connected regions together form a fundamental domain of the McKay quiver. So if $\mathrm{SS}_{\tilde{M}, E}$ has three sources $P_{1}, P_{2}, P_{3}$ as in Figure 25 the corresponding fundamental domain will be strictly contained in the fundamental domain defined by $\mathrm{SS}_{\tilde{\mathscr{M}}, F}$ (contradiction). 


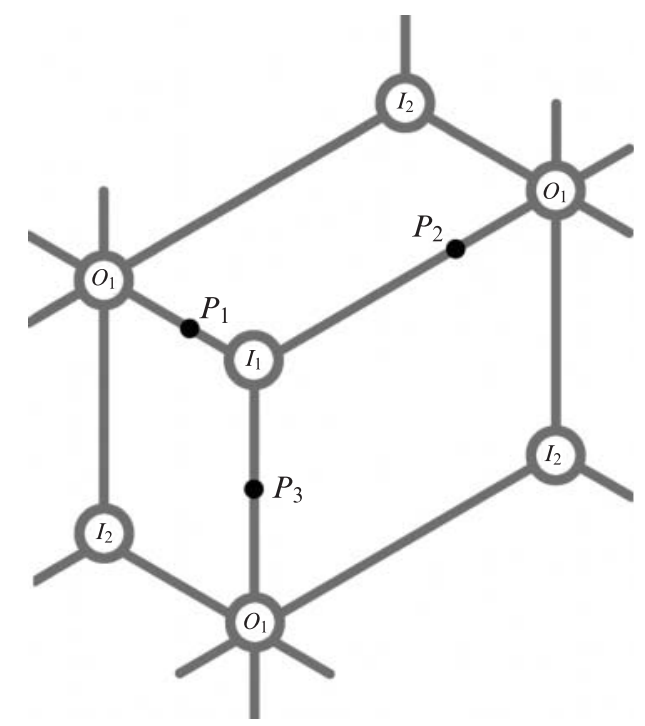

Figure 25. The sink-source graph $\mathrm{SS}_{\tilde{\mathscr{M}}, F}$ with sources $P_{1}, P_{2}$ and $P_{3}$ for $E$.

\section{Proof of the main results}

Proof of Theorem 1.1. Recall that $Y$ is $G-\operatorname{Hilb}\left(\mathbb{C}^{3}\right), \tilde{\mathscr{M}}$ is the dual of the universal family of $G$-clusters on $Y$ and $\Psi: D^{G}\left(\mathbb{C}^{3}\right) \rightarrow D(Y)$ is the integral transform defined by $\tilde{\mathscr{M}}$. We fix $\chi \in G^{\vee}$ and look at the associated representation $\mathrm{Q}(G)_{\tilde{\mu}}$ and its subrepresentation $\operatorname{Hex}\left(\chi^{-1}\right) \tilde{M}$. Then the total complex $T^{\bullet}$ of the skew-commutative cube corresponding to $\operatorname{Hex}\left(\chi^{-1}\right) \tilde{\mathscr{M}}$ gives $\Psi\left(\mathcal{O}_{0} \otimes \chi\right) \in D(Y)$ (Proposition 4.6).

If $\tilde{\mathscr{M}}=\bigoplus_{\chi \in G^{\vee}} \mathscr{L}\left(-M_{\chi}^{\prime}\right)$ then in the notation of Section 3, the skew-commutative cube $T^{\bullet}$ corresponding to $\operatorname{Hex}\left(\chi^{-1}\right) \tilde{\tilde{M}}$ is described as follows. For any subset $v$ of $\{1,2,3\}$ we have $\mathscr{L}_{v}=\mathscr{L}\left(-M_{\kappa(v) \chi}^{\prime}\right)$ where $\kappa(v)=\prod_{i \in v} \kappa\left(x_{i}\right)$. The divisors $D_{v}^{i}$ record where the maps $\alpha_{v}^{i}$ in the cube vanish (see Figure 26).

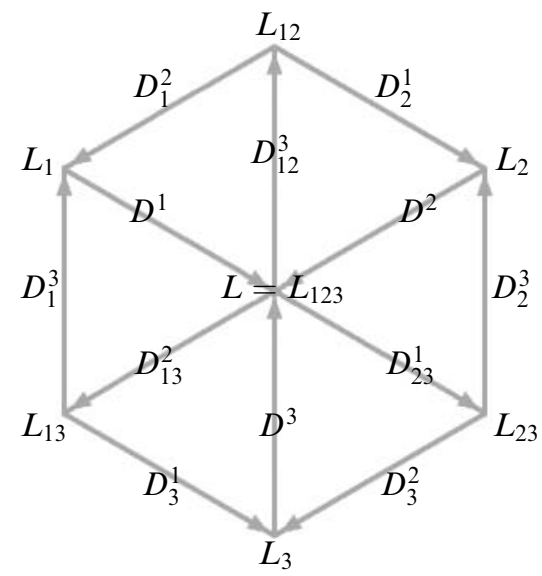

Figure 26. The skew-commutative cube corresponding to $\operatorname{Hex}\left(\chi^{-1}\right)$. 
By Lemma 3.1 the only way $H^{-2}\left(T^{\bullet}\right) \neq 0$ is if $\operatorname{gcd}\left(D_{23}^{1}, D_{13}^{2}, D_{12}^{3}\right) \neq 0$. This is only the case if $\chi=\chi_{0}$ (Corollary 4.13). On the other hand, if $\chi=\chi_{0}$, then $\chi$ is a $(0,3)$-source in $\mathrm{SS}_{\tilde{\mathscr{M}}, E}$ for every irreducible exceptional divisor $E$ on $Y$. So every $D_{j k}^{i}$ is equal to the whole exceptional set $\operatorname{Exc}(Y)$ while every $D_{j}^{i}$ and $D^{i}$ is zero. By Lemma 3.1 this means $H^{-2}\left(T^{\bullet}\right)=\mathscr{L}_{123}(\operatorname{Exc}(Y)) \otimes \mathcal{O}_{\operatorname{Exc}(Y)}$ and $H^{-1}\left(T^{\bullet}\right)=H^{0}\left(T^{\bullet}\right)=0$. Since $M_{\chi_{0}}^{\prime}=0$ we have $\mathscr{L}_{123}=\mathscr{L}\left(-M_{\chi_{0}}^{\prime}\right)=\mathcal{O}_{Y}$ and $\Psi\left(\mathcal{O}_{0} \otimes \chi\right)=\mathcal{O}(\operatorname{Exc}(Y)) \otimes \mathcal{O}_{\operatorname{Exc}(Y)}$.

For the rest of the argument we assume $\chi \neq \chi_{0}$ in which case $T^{\bullet}$ is supported in degrees 0 and -1 . We will need two facts. One is that

$$
\operatorname{Ext}^{0}\left(T^{\bullet}, T^{\bullet}\right) \cong \operatorname{Ext}_{G}^{0}\left(\mathcal{O}_{0} \otimes \chi, \mathcal{O}_{0} \otimes \chi\right) \cong \mathbb{C}
$$

since $\Psi$ is an equivalence. The other fact is the standard exact triangle

$$
\mathscr{W}[1] \rightarrow T^{\bullet} \rightarrow \mathcal{O}_{D^{1} \cap D^{2} \cap D^{3}}(\mathscr{L})
$$

where $\mathscr{W}=H^{-1}\left(T^{\bullet}\right)$.

Suppose $H^{0}\left(T^{\bullet}\right) \neq 0$. By Lemma 3.1 this means $D^{1} \cap D^{2} \cap D^{3} \neq 0$ where each $D^{i} \in \operatorname{Exc}(Y)$. Let $L$ be an irreducible component of $D^{1} \cap D^{2} \cap D^{3} \neq 0$. There are three possibilities:

(1) $L$ is an isolated point.

(2) $L$ is an intersection $E_{1} \cap E_{2}$ of two irreducible exceptional divisors.

(3) $L$ is an exceptional divisor.

Below we show that case $(1)$ cannot occur while in the other cases $\operatorname{Ext}^{1}\left(\mathcal{O}_{L} \otimes \mathscr{L}^{\prime}, \mathscr{W}[1]\right)=0$ for any line bundle $\mathscr{L}^{\prime}$. This implies $\operatorname{Ext}^{1}\left(\mathcal{O}_{D^{1} \cap D^{2} \cap D^{3}} \otimes \mathscr{L}, \mathscr{W}[1]\right)=0$ because we can repeatedly apply an exact sequence of the form

$$
\mathcal{O}_{L_{2}} \otimes \mathscr{I}_{L_{1}} \rightarrow \mathcal{O}_{L_{1} \cup L_{2}} \rightarrow \mathcal{O}_{L_{1}}
$$

where $L_{1}$ and $L_{2}$ are components of $D^{1} \cap D^{2} \cap D^{3}$. Notice that if we break up $D^{1} \cap D^{2} \cap D^{3}$ into components in the right order then we can ensure that $\mathcal{O}_{L_{2}} \otimes \mathscr{I}_{L_{1}}$ is a line bundle on $L_{2}$ so that by induction $\operatorname{Ext}^{1}\left(\mathcal{O}_{L_{2}} \otimes \mathscr{I}_{L_{1}}, \mathscr{W}[1]\right)=\operatorname{Ext}^{1}\left(\mathcal{O}_{L_{1}}, \mathscr{W}[1]\right)=0$ which implies $\operatorname{Ext}^{1}\left(\mathcal{O}_{L_{1} \cup L_{2}}, \mathscr{W}[1]\right)=0$.

The fact that $\operatorname{Ext}^{1}\left(\mathcal{O}_{D^{1} \cap D^{2} \cap D^{3}}(\mathscr{L}), \mathscr{W}[1]\right)=0$ implies $T^{\bullet} \cong \mathscr{W}[1] \oplus \mathcal{O}_{D^{1} \cap D^{2} \cap D^{3}}(\mathscr{L})$. This means $\mathscr{W}=0$ for otherwise $\operatorname{dim} \operatorname{Ext}^{0}\left(T^{\bullet}, T^{\bullet}\right) \geqq 2$. We conclude that if $H^{0}\left(T^{\bullet}\right) \neq 0$ then $H^{1}\left(T^{\bullet}\right)=0$. As by assumption $H^{i}\left(T^{\bullet}\right) \neq 0$ only for $i=0,-1$, this completes the proof of Theorem 1.1 . 
Case (1). The only way $D^{1} \cap D^{2} \cap D^{3}$ can contain an isolated point is if each $D^{i}$ contains an (irreducible) exceptional divisor $E_{i}$ such that $E_{1} \cap E_{2} \cap E_{3}$ is a point $p$. Then $H^{0}\left(T^{\bullet}\right)$ is the direct sum of $\mathcal{O}_{p}$ and some sheaf. If $p \notin \operatorname{Supp}(\mathscr{W})$ then $T^{\bullet}$ breaks up as the direct sum of $\mathcal{O}_{p}$ and some complex which contradicts the fact that $\operatorname{Ext}^{0}\left(T^{\bullet}, T^{\bullet}\right) \cong \mathbb{C}$. Thus $p$ belongs to the support of $\mathscr{W}$.

By Lemma 3.1 the support of $\mathscr{W}$ is contained in the union of $\operatorname{gcd}\left(D_{1}^{2}, D_{2}^{1}\right)$, $\operatorname{gcd}\left(D_{1}^{3}, D_{3}^{1}\right)$ and $\operatorname{gcd}\left(D_{2}^{3}, D_{3}^{2}\right)$. Without loss of generality suppose $p \in \operatorname{gcd}\left(D_{1}^{2}, D_{2}^{1}\right)$. Since $p$ belongs to precisely three exceptional divisors $\left(E_{1}, E_{2}\right.$ and $\left.E_{3}\right)$, one of them must belong to $\operatorname{gcd}\left(D_{1}^{2}, D_{2}^{1}\right)$. It cannot be $E_{1}$ or $E_{2}$ since $D^{1}+D_{1}^{2}=D^{2}+D_{2}^{1}$ contains $E_{1}+E_{2}$. Therefore $E_{3}$ belongs to $\operatorname{gcd}\left(D_{1}^{2}, D_{2}^{1}\right)$. This is impossible by Proposition 4.19 .

Case (2). Since $L=E_{1} \cap E_{2}$ is a component of $D^{1} \cap D^{2} \cap D^{3}$ we can assume (without loss of generality) that $D^{1}$ and $D^{2}$ contain $E_{1}$ while $D^{3}$ contains $E_{2}$. To show $\operatorname{Ext}^{1}\left(\mathcal{O}_{L} \otimes \mathscr{L}^{\prime}, \mathscr{W}[1]\right)=0$ it suffices to show that $\operatorname{Ext}^{2}\left(\mathcal{O}_{L} \otimes \mathscr{L}^{\prime}, \mathcal{O}_{C}\right)=0$ for any irreducible component $C$ of $\operatorname{Supp}(\mathscr{W})$. Denote by $i: C \rightarrow Y=G-\operatorname{Hilb}\left(\mathbb{C}^{3}\right)$ the inclusion. Then by adjunction

$$
\operatorname{Ext}^{2}\left(\mathcal{O}_{L} \otimes \mathscr{L}^{\prime}, \mathscr{O}_{C}\right) \cong \operatorname{Ext}^{2}\left(i^{*} \mathscr{O}_{L} \otimes \mathscr{L}^{\prime}, \mathcal{O}_{C}\right)
$$

so if $L \cap C=\emptyset$ then $i^{*} \mathscr{O}_{L}=0$ and we are done. So we can assume $L \cap C$ is nonempty.

Suppose $C \subset Y$ has codimension one (i.e. is an irreducible exceptional divisor). If $L \subset C$ then by Lemma 3.1 some $\operatorname{gcd}\left(D_{i}^{j}, D_{j}^{i}\right)$ must contain either $E_{1}$ or $E_{2}$. None of them can contain $E_{1}$ since $D^{1}$ and $D^{2}$ contain $E_{1}$ and so some $\operatorname{gcd}\left(D_{i}^{j}, D_{j}^{i}\right)$ must contain $E_{2}$. Since $D^{3}$ contains $E_{2}$ this means it must be $\operatorname{gcd}\left(D_{1}^{2}, D_{2}^{1}\right)$ that contains $E_{2}$. By Corollary 4.18 this implies $E_{1} \cap E_{2}=\emptyset$ (contradiction).

Suppose $L \cap C=E_{1} \cap E_{2} \cap C$ is zero dimensional (i.e. a point). By 3.1, some $\operatorname{gcd}\left(D_{i}^{j}, D_{j}^{i}\right)$ must contain $C$. By Corollary $4.18, \operatorname{gcd}\left(D_{1}^{2}, D_{2}^{1}\right)$ cannot contain $C$ since this would mean $C \cap E_{1}=\emptyset$. So, without loss of generality, we can assume $\operatorname{gcd}\left(D_{2}^{3}, D_{3}^{2}\right)$ contains $C$. Since $C \in \operatorname{supp} H^{-1}\left(T^{\bullet}\right)$, using Lemma 3.1, either $D^{1}$ contains $C$ or $\operatorname{lcm}\left(D_{1}^{2}, D_{1}^{3}\right)$ contains $C$.

If $D^{1}$ contains $C$ then by Proposition 4.19, $E_{1} \cap E_{2} \cap C=\emptyset$ (contradiction). So we assume $\operatorname{lcm}\left(D_{1}^{2}, D_{1}^{3}\right)$ contains $C$. Since neither $D^{1}$ nor $D^{2}$ contains $C$ this means either both $D_{1}^{2}$ and $D_{2}^{1}$ cannot $C$ or neither contain $C$. They cannot both contain $C$ because if they did then $C \cap E_{1}$ would be empty by Corollary 4.18. Thus $D_{1}^{2}$ does not contain $C$ and since $\operatorname{lcm}\left(D_{1}^{2}, D_{1}^{3}\right)$ contains $C$ this means $D_{1}^{3}$ must contain $C$. Finally, $D^{3}$ does not contain $C$ so this means $D_{3}^{1}$ must contain $C$. We conclude that $D_{2}^{3}, D_{3}^{2}, D_{1}^{3}$ and $D_{3}^{1}$ all contain $C$. By Proposition 4.20 this means $C \cap E_{1}=\emptyset$ (contradiction).

Finally, suppose $C \subset Y$ has codimension two so that $C=C_{1} \cap C_{2}$ where $C_{1}, C_{2} \in \operatorname{Exc}(Y)$. The fact $L \cap C$ is non-empty means that (without loss of generality) 
$C_{1}$ equals $E_{1}$ or $E_{2}$. Notice that $\operatorname{gcd}\left(D_{i}^{j}, D_{j}^{i}\right)$ cannot contain $C_{1}$ (to deal with $\operatorname{gcd}\left(D_{1}^{2}, D_{2}^{1}\right)$ we use 4.18). It follows by Lemma 3.1 that $C_{2}$ must be contained in some $\operatorname{gcd}\left(D_{i}^{j}, D_{j}^{i}\right)$. By $4.18, C_{2}$ cannot be contained in $\operatorname{gcd}\left(D_{1}^{2}, D_{2}^{1}\right)$ so, without loss of generality, let's suppose it is contained in $\operatorname{gcd}\left(D_{2}^{3}, D_{3}^{2}\right)$. By Lemma 3.1, $C_{1}$ must be contained in

$$
D^{1}+\operatorname{lcm}\left(D_{1}^{2}, D_{1}^{3}\right)-\tilde{D}_{2}^{3}-D^{2}
$$

since $C_{1} \cap C_{2} \in \operatorname{supp} H^{-1}\left(T^{\bullet}\right)$. Since $E_{1}$ belongs to $D^{2}$ this means $E_{1}$ cannot belong to $D^{1}+\operatorname{lcm}\left(D_{1}^{2}, D_{1}^{3}\right)-\tilde{D}_{2}^{3}-D^{2}$. So $C_{1}=E_{2}$. But then $E_{2}$ belongs to $\tilde{D}_{2}^{3}+D^{2}$ so again $C_{1}=E_{2}$ cannot belong to $D^{1}+\operatorname{lcm}\left(D_{1}^{2}, D_{1}^{3}\right)-\tilde{D}_{2}^{3}-D^{2}$ (contradiction).

Case (3). The only way $\operatorname{Ext}^{1}\left(\mathcal{O}_{L}(\mathscr{L}), \mathscr{W}[1]\right)$ is non-zero is if $L$ intersects (without loss of generality) $\operatorname{gcd}\left(D_{1}^{2}, D_{2}^{1}\right)$. Then $D^{1}$ and $D^{2}$ would contain $L$ while $\operatorname{gcd}\left(D_{1}^{2}, D_{2}^{1}\right)$ would contain an exceptional divisor $F$ such that $L \cap F \neq \emptyset$. This is impossible by Corollary 4.18 .

\section{A worked example}

In this section we give a worked example of how to compute images $\Psi\left(\mathcal{O}_{0} \otimes \chi\right)$ for a given abelian subgroup of $\mathrm{SL}_{3}(\mathbb{C})$.

6.1. The group $\boldsymbol{G}$ and its McKay quiver. We set $G$ to be the group $\frac{1}{13}(1,5,7)$. That is, the image in $\mathrm{SL}_{3}(\mathbb{C})$ of group $\mu_{13}$ of 13 th roots of unity under the embedding $\xi \mapsto\left(\begin{array}{ccc}\xi^{1} & & \\ & \xi^{5} & \\ & & \xi^{7}\end{array}\right)$. We denote by $\chi_{i}$ the character of $G$ induced by $\xi \mapsto \xi^{i}$. Then $\rho\left(x_{1}\right)=\chi_{1}, \rho\left(x_{2}\right)=\chi_{5}, \rho\left(x_{3}\right)=\chi_{7}$, therefore $\kappa\left(x_{1}\right)=\chi_{12}, \kappa\left(x_{2}\right)=\chi_{8}$ and $\kappa\left(x_{3}\right)=\chi_{6}$.

As explained in Section 4.1, for each $\chi_{i} \in G^{\vee}$ the McKay quiver $\mathrm{Q}(G)$ has 3 arrows emerging from the vertex $\chi_{i}$ and going to vertices $\kappa\left(x_{j}\right) \chi_{i}$ for $j=1,2,3$. E.g. from the vertex $\chi_{3}$ there are arrows going to vertices $\kappa\left(x_{1}\right) \chi_{3}=\chi_{2}, \kappa\left(x_{2}\right) \chi_{3}=\chi_{11}$ and $\kappa\left(x_{3}\right) \chi_{3}=\chi_{9}$. Choosing a particular fundamental domain in the planar embedding $\phi_{H}$ of the universal cover $U$ of $\mathrm{Q}(G)$ into $\mathbb{R}^{2}$, we can depict $\mathrm{Q}(G)$ as shown in Figure 27. Gluing together the edges of the diagram appropriately one obtains the real 2-dimensional torus $T_{G}$ as tessellated by the McKay quiver.

6.2. The resolution $G$-Hilb( $\left.\mathbb{C}^{3}\right)$ and the universal family $\mathscr{M}$ of $G$-clusters. Let $Y=G$-Hilb $\left(\mathbb{C}^{3}\right)$. For any abelian subgroup $G \subset \mathrm{SL}_{3}(\mathbb{C}), Y$ is a toric variety whose toric fan can be computed as described in [4], Section 2. In our case we obtain the 3dimensional fan in $\mathbb{R}^{3}$ as depicted in Figure 1. As usual, we drew the junior simplex $\Delta$ - the two-dimensional section of the fan cut out by the plane $l_{1}+l_{2}+l_{3}=1$. The generators $e_{i}$ of the one-dimensional cones of the fan all lie in $\Delta$ and have the following coordinates: 


$$
\begin{aligned}
& e_{1}=(1,0,0), \quad e_{2}=(0,1,0), \quad e_{3}=(0,0,1), \\
& e_{4}=\frac{1}{13}(1,5,7), \quad e_{5}=\frac{1}{13}(2,10,1), \quad e_{6}=\frac{1}{13}(3,2,8), \\
& e_{7}=\frac{1}{13}(4,7,2), \quad e_{8}=\frac{1}{13}(6,4,3), \quad e_{9}=\frac{1}{13}(8,1,4) \text {. }
\end{aligned}
$$

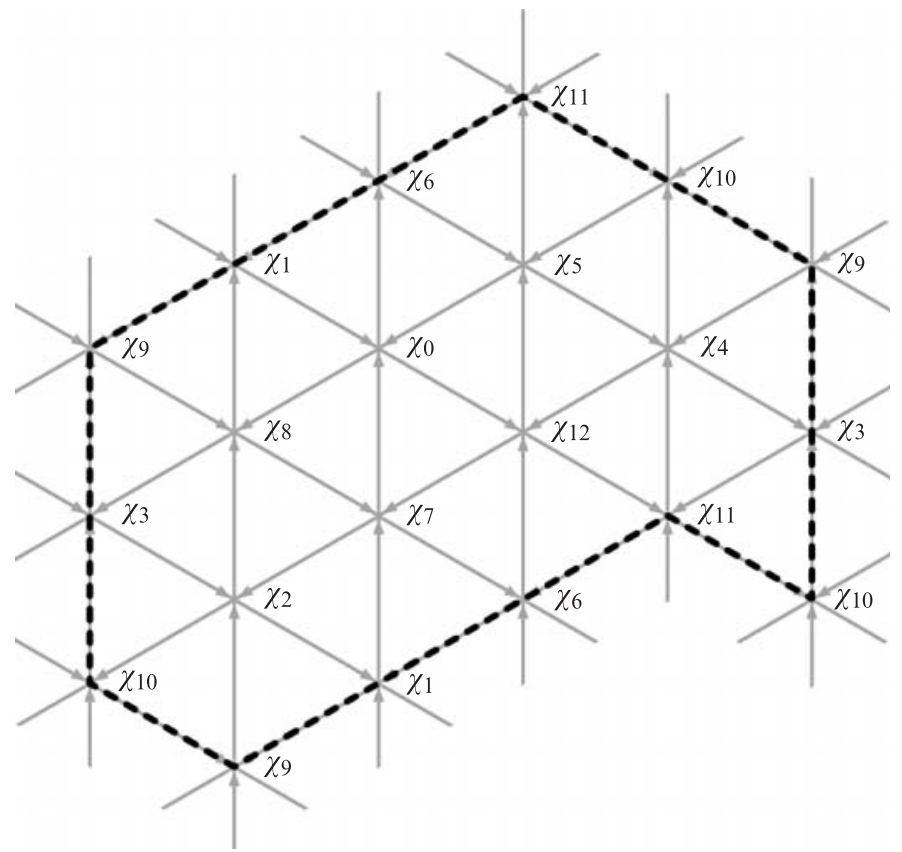

Figure 27. A fundamental domain of the McKay quiver of $G=\frac{1}{13}(1,5,7)$.

To each $k$-dimensional cone $\left\langle e_{i_{1}}, \ldots, e_{i_{k}}\right\rangle$ in the toric fan of $Y$ there corresponds a $(3-k)$-dimensional torus orbit in $Y$. We denote this orbit by $S_{i_{1}, \ldots, i_{k}}$ and its closure by $E_{i_{1}, \ldots, i_{k}}$. The set of all irreducible exceptional divisors on $Y$ is then $\left\{E_{4}, E_{5}, E_{6}, E_{7}, E_{8}, E_{9}\right\}$.

On $Y=G$-Hilb $\left(\mathbb{C}^{3}\right)$ the universal family $\mathscr{M}=\bigoplus \mathscr{L}\left(-M_{\chi}\right)$ of $G$-clusters $Y$ is the maximal shift gnat-family (cf. [10], Prop. 3.17). Each $G$-Weil divisor $M_{\chi}$ is of the form $\sum_{E_{i} \in \operatorname{Exc}(Y)} m_{\chi, i} E_{i}\left([10]\right.$, Prop. 3.15). As shown in [8], Example 4.21, the coefficients $m_{\chi, i} \in \mathbb{Q}$ can be computed via the formula

$$
m_{\chi, i}=\inf \left\{e_{i}(m) \mid m \in \mathbb{Z}_{\geqq 0}^{3} \text { and } \rho\left(x^{m}\right)=\chi\right\}
$$

To compute $m_{\chi, i}$ it suffices to test $e_{i}\left(m_{1}, m_{2}, m_{3}\right)$ for minimality only on $\left(m_{1}, m_{2}, m_{3}\right) \in \mathbb{Z}^{3}$ for which $0 \leqq m_{1}, m_{2}, m_{3} \leqq 13$. This is because $x_{1}^{13}, x_{2}^{13}$ and $x_{3}^{13}$ are $G$ invariant, while $e_{i}\left(m_{1}+13, m_{2}, m_{3}\right) \geqq e_{i}\left(m_{1}, m_{2}, m_{3}\right)$ and similarly for the other two. It then becomes a simple computer computation. 
For example, the minimal value of $e_{7}(-)$ on $\left(m_{1}, m_{2}, m_{3}\right)$ with $\rho\left(x_{1}^{m_{1}} x_{2}^{m_{2}} x_{3}^{m_{3}}\right)=\chi_{10}$ and $0 \leqq m_{1}, m_{2}, m_{3} \leqq 13$ can be verified to be $1 \frac{1}{13}$. E.g. $\rho\left(x_{1}^{3} x_{3}\right)=\chi_{1}^{3} \chi_{7}=\chi_{10}$ and

$$
e_{7}\left(x_{1}^{3} x_{3}\right)=e_{7}(3,0,1)=\frac{4}{13} \cdot 3+\frac{7}{13} \cdot 0+\frac{2}{13} \cdot 1=1 \frac{1}{13} .
$$

Hence $m_{\chi_{10}, 7}=1 \frac{1}{13}$.

Repeating this for other $\chi$ and $e_{i}$, we compute $m_{\chi, i}$ to be:

\begin{tabular}{|c|c|c|c|c|c|c||c|c|c|c|c|c|c|}
\hline$\chi \backslash i$ & 4 & 5 & 6 & 7 & 8 & 9 & $\chi \backslash i$ & 4 & 5 & 6 & 7 & 8 & 9 \\
\hline$\chi_{0}$ & 0 & 0 & 0 & 0 & 0 & 0 & $\chi_{7}$ & $\frac{7}{13}$ & $\frac{1}{13}$ & $\frac{8}{13}$ & $\frac{2}{13}$ & $\frac{3}{13}$ & $\frac{4}{13}$ \\
$\chi_{1}$ & $\frac{1}{13}$ & $\frac{2}{13}$ & $\frac{3}{13}$ & $\frac{4}{13}$ & $\frac{6}{13}$ & $\frac{8}{13}$ & $\chi_{8}$ & $\frac{8}{13}$ & $\frac{3}{13}$ & $\frac{11}{13}$ & $\frac{6}{13}$ & $\frac{9}{13}$ & $\frac{12}{13}$ \\
$\chi_{2}$ & $\frac{2}{13}$ & $\frac{4}{13}$ & $\frac{6}{13}$ & $\frac{8}{13}$ & $\frac{12}{13}$ & $\frac{3}{13}$ & $\chi_{9}$ & $\frac{9}{13}$ & $\frac{5}{13}$ & $1 \frac{1}{13}$ & $\frac{10}{13}$ & $1 \frac{2}{13}$ & $\frac{7}{13}$ \\
$\chi_{3}$ & $\frac{3}{13}$ & $\frac{6}{13}$ & $\frac{9}{13}$ & $\frac{12}{13}$ & $1 \frac{5}{13}$ & $\frac{11}{13}$ & $\chi_{10}$ & $\frac{10}{13}$ & $\frac{7}{13}$ & $\frac{4}{13}$ & $1 \frac{1}{13}$ & $\frac{8}{13}$ & $\frac{2}{13}$ \\
$\chi_{4}$ & $\frac{4}{13}$ & $\frac{8}{13}$ & $\frac{12}{13}$ & $\frac{3}{13}$ & $\frac{11}{13}$ & $\frac{6}{13}$ & $\chi_{11}$ & $\frac{11}{13}$ & $\frac{9}{13}$ & $\frac{7}{13}$ & $1 \frac{5}{13}$ & $1 \frac{1}{13}$ & $\frac{10}{13}$ \\
$\chi_{5}$ & $\frac{5}{13}$ & $\frac{10}{13}$ & $\frac{2}{13}$ & $\frac{7}{13}$ & $\frac{4}{13}$ & $\frac{1}{13}$ & $\chi_{12}$ & $\frac{12}{13}$ & $\frac{11}{13}$ & $\frac{10}{13}$ & $\frac{9}{13}$ & $\frac{7}{13}$ & $\frac{5}{13}$ \\
$\chi_{6}$ & $\frac{6}{13}$ & $\frac{12}{13}$ & $\frac{5}{13}$ & $\frac{11}{13}$ & $\frac{10}{13}$ & $\frac{9}{13}$ & & & & & & & \\
\hline
\end{tabular}

6.3. Divisors of the associated representation of the dual family $\tilde{\mathscr{M}}$. By Proposition 2.4 , the dual $\tilde{\mathscr{M}}$ of $\mathscr{M}=\bigoplus\left(-M_{\chi}\right)$ is the family $\bigoplus \mathscr{L}\left(-M_{\chi}^{\prime}\right)$ where $M_{\chi}^{\prime}=-M_{\chi^{-1}}$. So to compute the divisors of zeroes $B_{\chi, i}$ of the maps $\alpha_{\chi, i}$ in the associated representation of the McKay quiver $\mathrm{Q}(G)_{\tilde{\mathscr{M}}}$ we must set $D_{\chi}=-M_{\chi^{-1}}$ in formula (4.5). Thus we obtain the formula

$$
B_{\chi, i}=M_{\chi \rho\left(x_{i}\right)^{-1}}+\left(x_{i}\right)-M_{\chi} .
$$

The principal $G$-Weil divisors $\left(x_{i}\right)$ of the basic monomials can be computed via the formula $\left(x_{i}\right)=\sum e_{i}\left(x_{i}\right) E_{i}$ (cf. [8], Prop. 3.2). For an example, $e_{4}=\frac{1}{13}(1,5,7)$ so $e_{4}\left(x_{1}\right)=\frac{1}{13}, e_{4}\left(x_{2}\right)=\frac{5}{13}$ and $e_{4}\left(x_{3}\right)=\frac{7}{13}$ and obtain:

$$
\begin{aligned}
& \left(x_{1}\right)=E_{1}+\frac{1}{13} E_{4}+\frac{2}{13} E_{5}+\frac{3}{13} E_{6}+\frac{4}{13} E_{7}+\frac{6}{13} E_{8}+\frac{8}{13} E_{9}, \\
& \left(x_{2}\right)=E_{2}+\frac{5}{13} E_{4}+\frac{10}{13} E_{5}+\frac{2}{13} E_{6}+\frac{7}{13} E_{7}+\frac{4}{13} E_{8}+\frac{1}{13} E_{9}, \\
& \left(x_{3}\right)=E_{3}+\frac{7}{13} E_{4}+\frac{1}{13} E_{5}+\frac{8}{13} E_{6}+\frac{2}{13} E_{7}+\frac{3}{13} E_{8}+\frac{4}{13} E_{9} .
\end{aligned}
$$


Let us now compute, for example, zero divisor $B_{\chi_{5}, 1}$. Using formula (6.4) we have

$$
\begin{aligned}
B_{\chi_{5}, 1}= & M_{\chi_{5} \chi_{1}^{-1}}+\left(x_{1}\right)+M_{\chi_{5}}=M_{\chi_{4}}+\left(x_{1}\right)-M_{\chi_{5}} \\
= & \frac{4}{13} E_{4}+\frac{8}{13} E_{5}+\frac{12}{13} E_{6}+1 \frac{3}{13} E_{7}+\frac{11}{13} E_{8}+\frac{6}{13} E_{9} \\
& +E_{1}+\frac{1}{13} E_{4}+\frac{2}{13} E_{5}+\frac{3}{13} E_{6}+\frac{4}{13} E_{7}+\frac{6}{13} E_{8}+\frac{8}{13} E_{9} \\
& \quad-\frac{5}{13} E_{4}-\frac{10}{13} E_{5}-\frac{2}{13} E_{6}-\frac{7}{13} E_{7}-\frac{4}{13} E_{8}-\frac{1}{13} E_{9} \\
= & \quad E_{1} \quad E_{6}+E_{7}+E_{8}+E_{9} .
\end{aligned}
$$

Computing similarly the remaining zero divisors $B_{\chi, k}$ of $\mathrm{Q}(G)_{\tilde{M}}$ we obtain:

$$
\begin{array}{ll}
B_{\chi_{0}, 1}=E_{1}+E_{4}+E_{5}+E_{6}+E_{7}+E_{8}+E_{9}, & B_{\chi_{7}, 1}=E_{1}+E_{5}+E_{7}+E_{8}+E_{9}, \\
B_{\chi_{0}, 2}=E_{2}+E_{4}+E_{5}+E_{6}+E_{7}+E_{8}+E_{9}, & B_{\chi_{7}, 2}=E_{2}+E_{5}+E_{7}+E_{8}, \\
B_{\chi_{0}, 3}=E_{3}+E_{4}+E_{5}+E_{6}+E_{7}+E_{8}+E_{9}, & B_{\chi_{7}, 3}=E_{3}, \\
B_{\chi_{1}, 1}=E_{1}, & B_{\chi_{8}, 1}=E_{1}, \\
B_{\chi_{1}, 2}=E_{2}+E_{4}+E_{5}+E_{6}+E_{7}+E_{8}, & B_{\chi_{8}, 2}=E_{2}+E_{5}+E_{7}+E_{8}, \\
B_{\chi_{1}, 3}=E_{3}+E_{4}+E_{6}, & B_{\chi_{8}, 3}=E_{3}, \\
B_{\chi_{2}, 1}=E_{1}+E_{9}, & B_{\chi_{9}, 1}=E_{1}+E_{9}, \\
B_{\chi_{2}, 2}=E_{2}+E_{4}+E_{5}+E_{7}, & B_{\chi_{9}, 2}=E_{2}+E_{5}+E_{7}, \\
B_{\chi_{2}, 3}=E_{3}+E_{4}+E_{6}+E_{9}, & B_{\chi_{9}, 3}=E_{3}, \\
B_{\chi_{3}, 1}=E_{1}, & B_{\chi_{10}, 1}=E_{1}+E_{6}+E_{8}+E_{9}, \\
B_{\chi_{3}, 2}=E_{2}+E_{4}+E_{5}+E_{7}, & B_{\chi_{10}, 2}=E_{2}+E_{5}, \\
B_{\chi_{3}, 3}=E_{3}+E_{4}+E_{6}, & B_{\chi_{10}, 3}=E_{3}+E_{6}+E_{8}+E_{9}, \\
B_{\chi_{4}, 1}=E_{1}+E_{8}+E_{9}, & B_{\chi_{11}, 1}=E_{1}, \\
B_{\chi_{4}, 2}=E_{2}+E_{4}+E_{5}, & B_{\chi_{11}, 2}=E_{2}+E_{5}, \\
B_{\chi_{4}, 3}=E_{3}+E_{4}, & B_{\chi_{11}, 3}=E_{3}+E_{6}, \\
B_{\chi_{5}, 1}=E_{1}+E_{6}+E_{7}+E_{8}+E_{9}, & B_{\chi_{12}, 1}=E_{1}+E_{7}+E_{8}+E_{9}, \\
B_{\chi_{5}, 2}=E_{2}, & B_{\chi_{12}, 2}=E_{2}, \\
B_{\chi_{5}, 3}=E_{3}+E_{4}+E_{6}+E_{7}+E_{8}+E_{9}, & B_{\chi_{12}, 3}=E_{3} . \\
B_{\chi_{6}, 1}=E_{1}, & \\
B_{\chi_{6}, 2}=E_{2}, & \\
B_{\chi_{6}, 3}=E_{3}+E_{4}+E_{6}, & \\
& \\
& \\
&
\end{array}
$$


6.4. Skew-commutative cubes corresponding to $\Psi\left(\mathcal{O}_{0} \otimes \chi\right)$. Now, for each $\chi \in G^{\vee}$, we compute the skew-commutative cube of line bundles $\operatorname{Hex}\left(\chi^{-1}\right) \tilde{\mathscr{M}}$ (see Section 4.2). The total complex of the cube gives then $\Psi\left(\mathcal{O}_{0} \otimes \chi\right)$ in $D(Y)$.

Recall that the subquiver $\operatorname{Hex}\left(\chi^{-1}\right)$ was defined by sending vertex $v \subset\{1,2,3\}$ of the cube quiver to vertex $\kappa(v)^{-1} \chi^{-1}$ in $\mathrm{Q}(G)$. Therefore at vertex $v$ of the cube of line bundles corresponding to $\operatorname{Hex}\left(\chi^{-1}\right)_{\tilde{M}}$ we get the summand of $\tilde{\mathscr{M}}=\bigoplus \mathscr{L}\left(-M_{\gamma}^{\prime}\right)$ which $G$ acts on by $\kappa(v)^{-1} \chi^{-1}$. So $\mathscr{L}_{v}=\mathscr{L}\left(-M_{\kappa(v) \chi}^{\prime}\right)=\mathscr{L}\left(M_{(\kappa(v) \chi)^{-1}}\right)$. On each arrow in the cube we mark its divisor of the zeroes, using the data of (6.5): $D_{j k}^{i}=B_{\chi^{-1}, i}, D_{j}^{i}=B_{\kappa\left(x_{k}\right) \chi^{-1, j}}$ and $D^{i}=B_{\kappa\left(x_{i}\right)^{-1} \chi^{-1, i}}$ for any $i \neq j \neq k$. We use a following shorthand: $E_{456}=E_{4}+E_{5}+E_{6}$ et cetera.

$\Psi\left(\mathcal{O}_{0} \otimes \chi_{0}\right):$

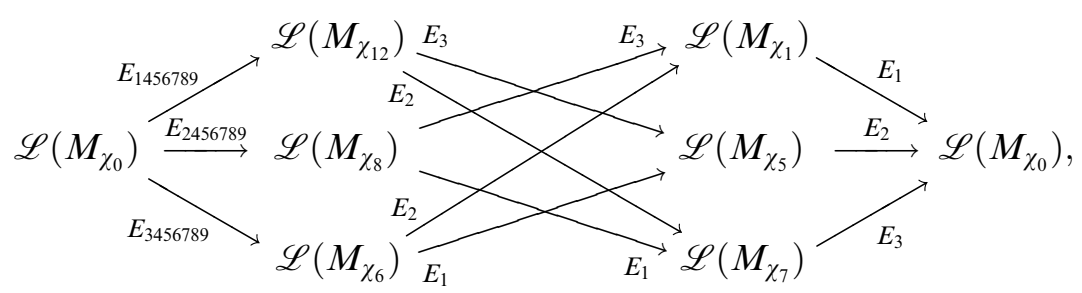

$\Psi\left(\mathcal{O}_{0} \otimes \chi_{1}\right):$

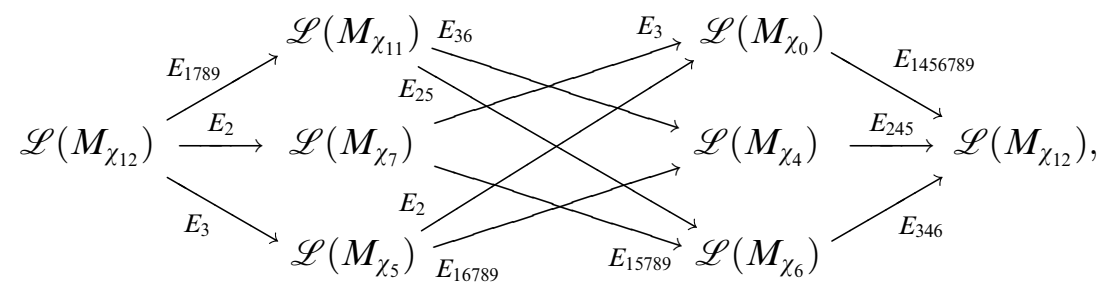

$\Psi\left(\mathcal{O}_{0} \otimes \chi_{2}\right):$

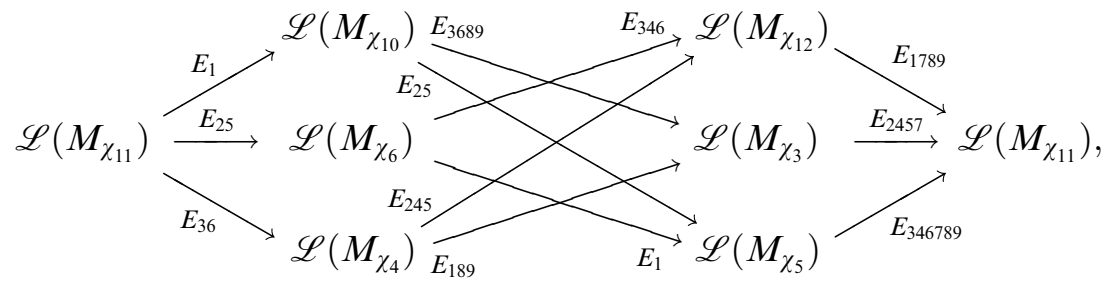

$\Psi\left(\mathcal{O}_{0} \otimes \chi_{3}\right):$

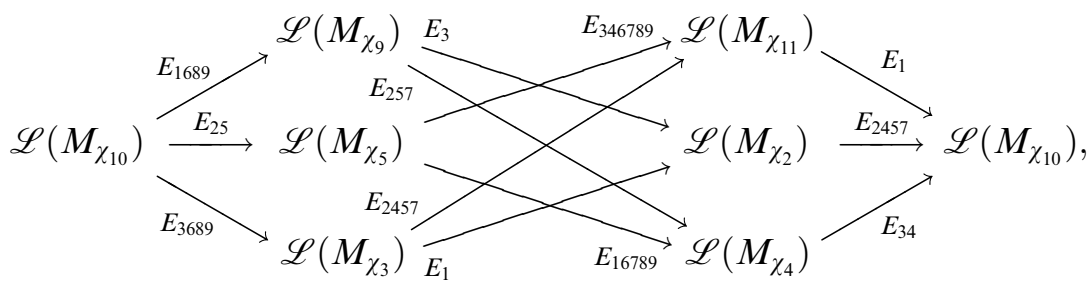

$\Psi\left(\mathcal{O}_{0} \otimes \chi_{4}\right):$

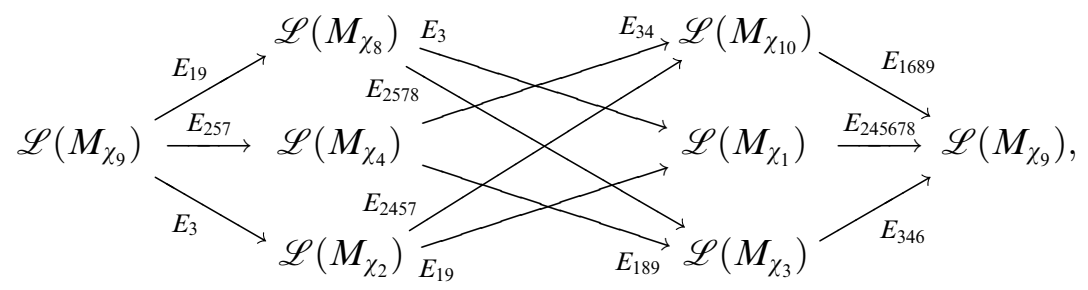


$\Psi\left(\mathcal{O}_{0} \otimes \chi_{5}\right)$

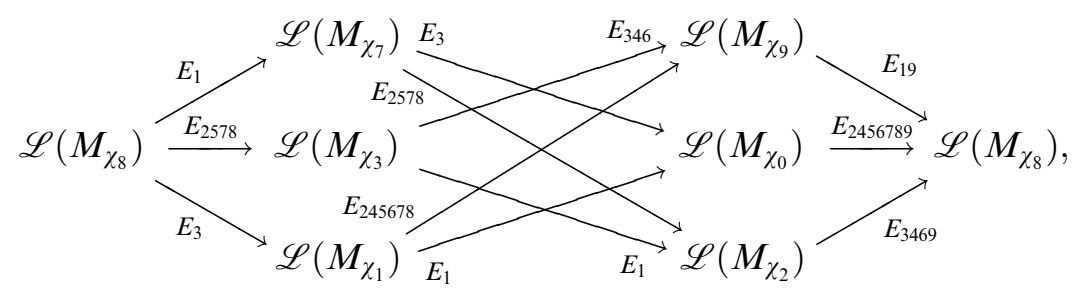

$\Psi\left(\mathcal{O}_{0} \otimes \chi_{6}\right)$

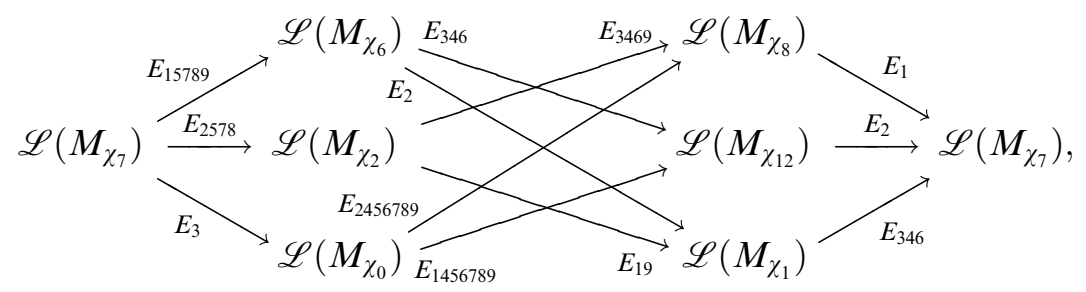

$\Psi\left(\mathcal{O}_{0} \otimes \chi_{7}\right)$

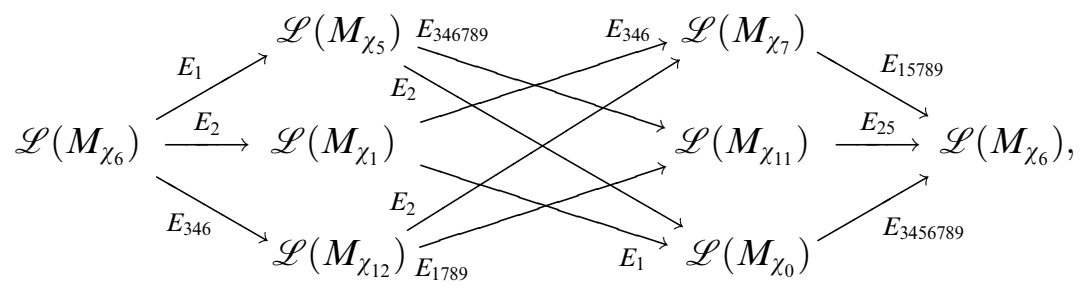

$\Psi\left(\mathcal{O}_{0} \otimes \chi_{8}\right)$

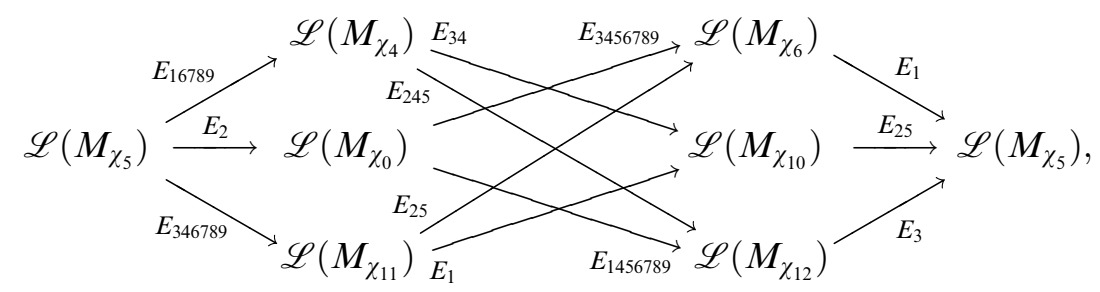

$\Psi\left(\mathcal{O}_{0} \otimes \chi_{9}\right)$

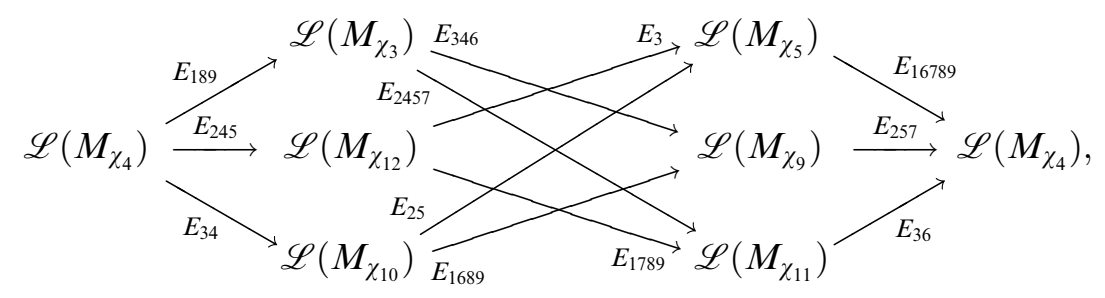

$\Psi\left(\mathcal{O}_{0} \otimes \chi_{10}\right)$

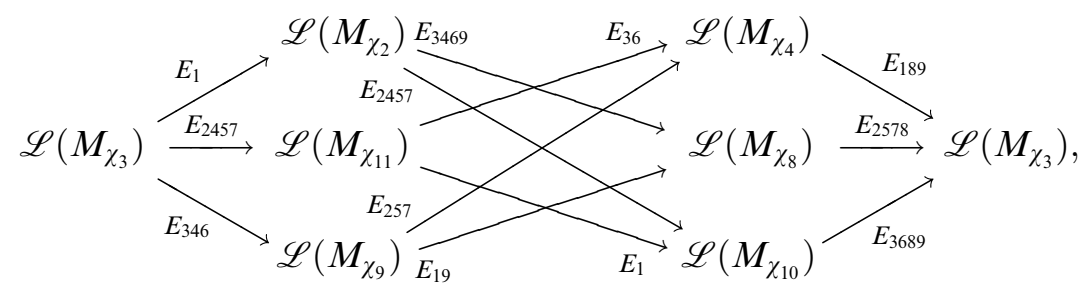

$\Psi\left(\mathcal{O}_{0} \otimes \chi_{11}\right)$

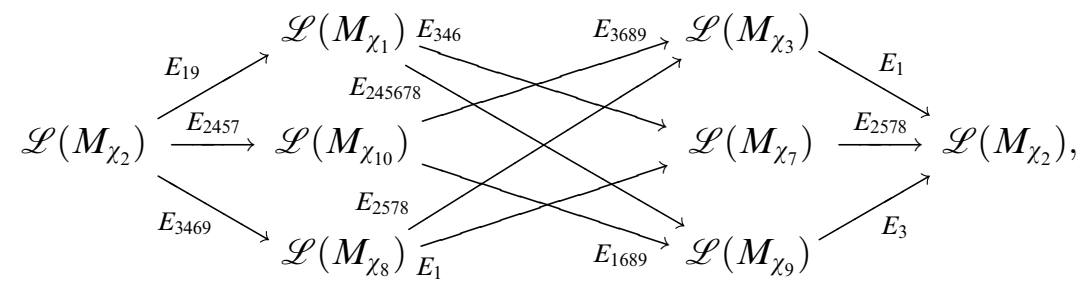


$\Psi\left(\mathcal{O}_{0} \otimes \chi_{12}\right):$

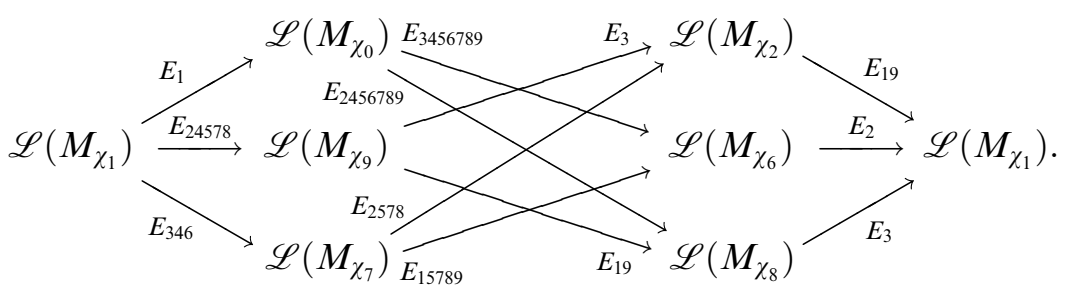

6.5. Conclusion. We now analyze each of the skew-commutative cubes computed in the previous section and use Lemma 3.1 to write down supports of the cohomology sheaves of their total complexes, i.e. of $\Psi\left(\mathcal{O}_{0} \otimes \chi\right)$.

For example, consider the skew-commutative cube for $\Psi\left(\mathcal{O}_{0} \otimes \chi_{1}\right)$ :

$$
\Psi\left(\mathcal{O}_{0} \otimes \chi_{1}\right)
$$

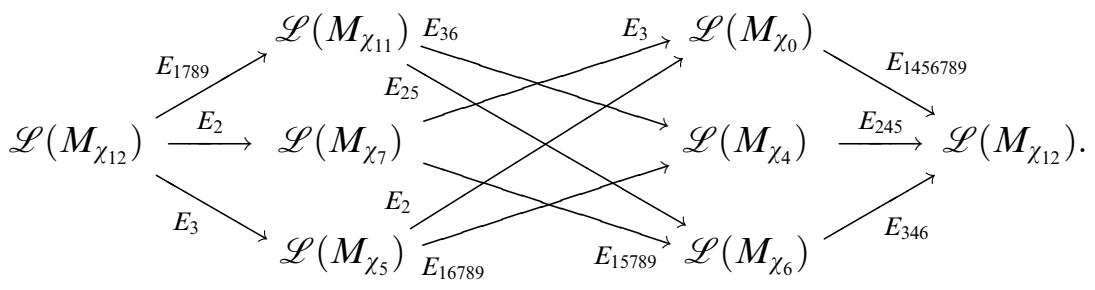

We see that $D^{1} \cap D^{2} \cap D^{3}=E_{4}$, that $\operatorname{gcd}\left(D_{2}^{1}, D_{1}^{2}\right)=\operatorname{gcd}\left(D_{3}^{2}, D_{2}^{3}\right)=\operatorname{gcd}\left(D_{3}^{1}, D_{1}^{3}\right)=0$ and that $\operatorname{gcd}\left(D_{23}^{1}, D_{13}^{2}, D_{12}^{3}\right)=0$. Therefore by Lemma 3.1 we have

$$
\operatorname{Supp} H^{0}\left(\Psi\left(\mathcal{O}_{0} \otimes \chi_{1}\right)\right)=E_{4}
$$

while Supp $H^{-1}\left(\Psi\left(\mathcal{O}_{0} \otimes \chi_{1}\right)\right)=\operatorname{Supp} H^{-2}\left(\Psi\left(\mathcal{O}_{0} \otimes \chi_{1}\right)\right)=0$.

On the other hand, looking at the skew-commutative cube for $\Psi\left(\mathcal{O}_{0} \otimes \chi_{11}\right)$ :

$\Psi\left(\mathcal{O}_{0} \otimes \chi_{11}\right)$

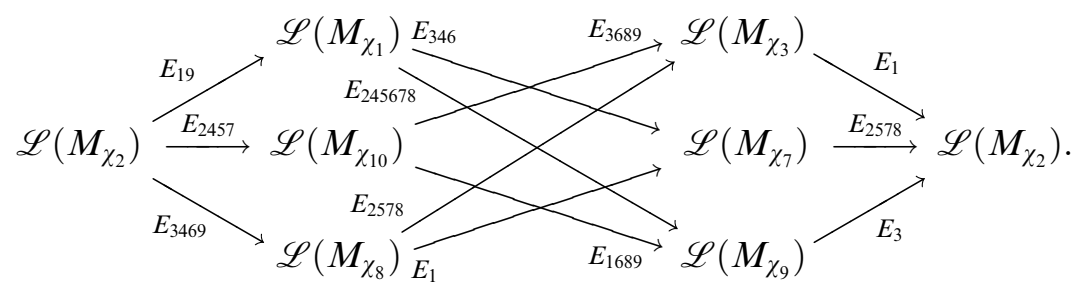

We see that $D^{1} \cap D^{2} \cap D^{3}=0$ and $\operatorname{gcd}\left(D_{23}^{1}, D_{13}^{2}, D_{12}^{3}\right)=0$, while $\operatorname{gcd}\left(D_{2}^{1}, D_{1}^{2}\right)=0$, $\operatorname{gcd}\left(D_{3}^{2}, D_{2}^{3}\right)=E_{46}, \operatorname{gcd}\left(D_{3}^{1}, D_{1}^{3}\right)=E_{689}$. Computing further

$$
D^{1}+\operatorname{lcm}\left(D_{1}^{2}, D_{1}^{3}\right)-\tilde{D}_{2}^{3}-D^{2}=E_{1}+E_{2356789}-E_{3}-E_{2578}=E_{169}
$$

and similarly

$$
D^{2}+\operatorname{lcm}\left(D_{2}^{1}, \tilde{D}_{2}^{3}\right)-\tilde{D}_{3}^{1}-D^{3}=E_{2578}+E_{13}-E_{1}-E_{3}=E_{2578}
$$

Consulting Figure 1 to compute intersections we see that

$$
\operatorname{gcd}\left(D_{3}^{2}, D_{2}^{3}\right) \cap\left(D^{1}+\operatorname{lcm}\left(D_{1}^{2}, D_{1}^{3}\right)-\tilde{D}_{2}^{3}-D^{2}\right)=E_{46} \cap E_{169}=E_{6}
$$


and

$$
\operatorname{gcd}\left(D_{3}^{1}, D_{1}^{3}\right) \cap\left(D^{2}+\operatorname{lcm}\left(D_{2}^{1}, \tilde{D}_{2}^{3}\right)-\tilde{D}_{3}^{1}-D^{3}\right)=E_{689} \cap E_{2578}=E_{8}
$$

Therefore by Lemma 3.1 we have Supp $H^{-1}\left(\Psi\left(\mathcal{O}_{0} \otimes \chi_{11}\right)\right)=E_{6} \cup E_{8}$ while

$$
\operatorname{Supp} H^{0}\left(\Psi\left(\mathcal{O}_{0} \otimes \chi_{11}\right)\right)=\operatorname{Supp} H^{-2}\left(\Psi\left(\mathcal{O}_{0} \otimes \chi_{11}\right)\right)=0
$$

Computing thus for each $\Psi\left(\mathcal{O}_{0} \otimes \chi\right)$, we obtain finally the data presented in Table 1.3 in the Introduction.

\section{Appendix A. Locally free resolutions of finite-length sheaves on $\mathbb{C}^{n}$}

In this section we present a general method to resolve any finite-length sheaf on $\mathbb{C}^{n}$ by locally free sheaves. It is sufficiently intrinsic to lift to a relative setup of a flat family of finite-length sheaves over a scheme of finite type over $\mathbb{C}$. This result may be of independent interest.

We then use this result to give an alternate proof of Proposition 2.1 where we use this resolution to explicitly compute the dual $\mathscr{F}^{\vee}$ in $D(Y)$.

A.1. Resolving finite-length sheaves on $\mathbb{C}^{n}$ by locally frees. The first step is the following technical lemma:

Let $A$ be a Hopf $\mathbb{C}$-algebra with a bijective antipode map. Denote by $\Delta$ its comultiplication map, by $\varepsilon$ the counit and by $S$ the antipode map. Let $W$ be a left $A$-module, which is finitely generated as a $\mathbb{C}$-module. Denote by $M_{W}$ the left $A$-module structure on the $\mathbb{C}$ module $A \otimes W$ given by, in Sweedler's sigma notation ([14], Section 1.2):

$$
a \cdot(b \otimes w)=\sum b S\left(a_{1}\right) \otimes a_{2} \cdot w .
$$

Denote by $M_{W}^{\prime}$ the structure of a free left $A$-module on $A \otimes W$ where $A$ acts on the first factor of the tensor product by left multiplication.

Denote by $\alpha$ the surjective $\mathbb{C}$-module map $A \otimes W \rightarrow W$ given by $a \otimes w \mapsto a \cdot w$. Denote by $\beta$ the surjective map $A \otimes W \rightarrow \mathbb{C} \otimes_{A} M_{W}$, where the $A$-module structure on $\mathbb{C}$ being given by the counit. We claim that $\alpha$ filters through $\beta$. Indeed, the kernel of $\beta$ is generated by the elements of form

$$
\sum\left(b S\left(a_{1}\right) \otimes a_{2} \cdot w\right)-\varepsilon(a) b \otimes w
$$

for all $a, b \in A$ and $w \in W$. Rewriting as

$$
\begin{aligned}
& \sum\left(b S\left(a_{1}\right) \otimes a_{2} \cdot w\right)-b \otimes\left(\sum S\left(a_{1}\right) a_{2}\right) \cdot w \\
& \quad=\sum\left(b S\left(a_{1}\right) \otimes a_{2} \cdot w-b \otimes\left(S\left(a_{1}\right) a_{2}\right) \cdot w\right)
\end{aligned}
$$


we see that each such element lies also in the kernel of $\alpha$. We thus have a commutative diagram:

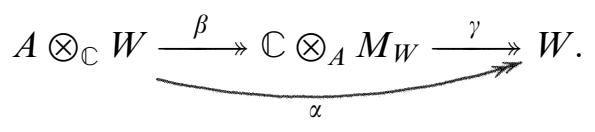

Lemma A.1. (1) $M_{W} \simeq M_{W}^{\prime}$ as left A-modules.

(2) The map $\gamma$ in (A.1) is a $\mathbb{C}$-module isomorphism.

Proof. (1) The isomorphism is given by the map $M_{W}^{\prime} \rightarrow M_{W}$ which sends $a \otimes w$ to $\sum S\left(a_{1}\right) \otimes a_{2} \cdot w$. Its inverse is the map $M_{W} \rightarrow M_{W}^{\prime}$ which sends $a \otimes w$ to $\sum S^{-1}\left(a_{2}\right) \otimes a_{1} \cdot w$. This can be verified using [14], Prop. 4.0.1, identities (3) and (4), and the identity (4) (iv) of the Exercises which immediately follow.

(2) From (1) we have that $M_{W}$ is free. Hence $\mathbb{C} \otimes_{A} M_{W}$ is isomorphic to $W$ as a $\mathbb{C}$-module. As $\mathbb{C} \otimes_{A} M_{W} \stackrel{\gamma}{\rightarrow} W$ is surjective it must also be an isomorphism.

Let $\mathscr{W}$ be a finite length $G$-sheaf on $\mathbb{C}^{n}$. Then $\Gamma(\mathscr{W})$ is a finite dimensional vector space with a $G$-action. Denote by $\mathscr{L}$ the locally free $G$-sheaf $\mathcal{O}_{\mathbb{C}^{n}} \otimes \Gamma(\mathscr{W})$. Its $\mathcal{O}_{\mathbb{C}^{n}}$-module structure is the natural action on the first factor of the tensor product, while the action of $G$ is on both the factors.

Proposition A.2. $\quad \mathscr{W}$ has a G-equivariant locally free resolution given by

$$
0 \longrightarrow \wedge^{n} V_{\text {giv }} \otimes \mathscr{L} \stackrel{\delta_{n}}{\longrightarrow} \cdots \stackrel{\delta_{k+1}}{\longrightarrow} \wedge^{k} V_{\text {giv }} \otimes \mathscr{L} \stackrel{\delta_{k}}{\longrightarrow} \cdots \stackrel{\delta_{1}}{\longrightarrow} \mathscr{L} \stackrel{\alpha}{\longrightarrow} \mathscr{W} \longrightarrow 0
$$

where the maps $\delta_{k}$ are defined for $m_{i} \in V_{\text {giv }}^{\vee}, f \in \mathcal{O}_{\mathbb{C}^{n}}$ and $w \in \Gamma(\mathscr{W})$ by

$$
\begin{aligned}
& \left(m_{1} \wedge \cdots \wedge m_{k}\right) f \otimes w \\
& \quad \mapsto \sum_{i=1}^{k}(-1)^{i+1}\left(\ldots m_{i-1} \wedge m_{i+1} \ldots\right)\left(f \otimes m_{i} \cdot w-m_{i} f \otimes w\right)
\end{aligned}
$$

and the map $\alpha$ is defined by $f \otimes w \mapsto f \cdot w$.

Proof. Let $\mathscr{L}^{\prime}$ be a $G$-sheaf on $\mathbb{C}^{n}$, which has the same underlying sheaf of $\mathbb{C}$ -

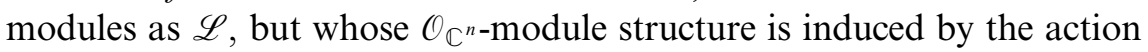

$$
m \cdot f \otimes w=f \otimes m \cdot w-m f \otimes w .
$$

of $V_{\text {giv }}^{\vee}$. The action of $G$ remains the same.

It is easy to check that the differential maps $\delta_{k}$ of the complex (A.2) respect this new $G$-sheaf structure. The resulting complex

$$
0 \longrightarrow \wedge^{n} V_{\text {giv }} \otimes \mathscr{L}^{\prime} \stackrel{\delta_{n}}{\longrightarrow} \cdots \stackrel{\delta_{k+1}}{\longrightarrow} \wedge^{k} V_{\text {giv }} \otimes \mathscr{L}^{\prime} \stackrel{\delta_{k}}{\longrightarrow} \cdots \stackrel{\delta_{1}}{\longrightarrow} \mathscr{L}^{\prime} \longrightarrow 0
$$

is precisely the Koszul complex of $\mathscr{L}^{\prime}$ with respect to any basis of $V_{\text {giv }}^{\vee}$. Let $m$ be the ideal sheaf of $\mathcal{O}_{\mathbb{C}^{n}}$ generated by the elements of $V_{\text {giv }}^{\vee}$. For grossly general reasons, as can be seen 
from Lemma A.1 (1), $\mathscr{L}^{\prime}$ is locally free. In particular, then, it is a Cohen-Macaulay

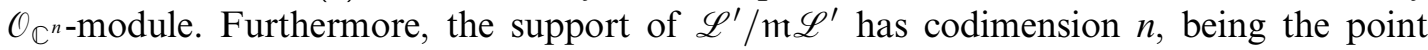
$0 \in \mathbb{C}^{n}$. Any basis of $V_{\text {giv }}^{\vee}$, as it consists of $n$ elements and generates $m$, must therefore be a regular sequence for $\mathscr{L}^{\prime}$. Hence the $G$-sheaf complex

$$
0 \longrightarrow \wedge^{n} V_{\text {giv }} \otimes \mathscr{L}^{\prime} \stackrel{\delta_{n}}{\longrightarrow} \cdots \stackrel{\delta_{k+1}}{\longrightarrow} \wedge^{k} V_{\text {giv }} \otimes \mathscr{L}^{\prime} \stackrel{\delta_{k}}{\longrightarrow} \cdots \stackrel{\delta_{1}}{\longrightarrow} \mathscr{L}^{\prime} \stackrel{\beta}{\longrightarrow} \mathscr{L}^{\prime} / \mathrm{m} \mathscr{L}^{\prime} \longrightarrow 0
$$

is exact. It then follows from Lemma A.1 (2), that the following is also exact as a complex of sheaves of $\mathbb{C}$-modules:

$$
0 \longrightarrow \wedge^{n} V_{\text {giv }} \otimes \mathscr{L}^{\prime} \stackrel{\delta_{n}}{\longrightarrow} \cdots \stackrel{\delta_{k+1}}{\longrightarrow} \wedge^{k} V_{\text {giv }} \otimes \mathscr{L}^{\prime} \stackrel{\delta_{k}}{\longrightarrow} \cdots \stackrel{\delta_{1}}{\longrightarrow} \mathscr{L}^{\prime} \stackrel{\alpha}{\longrightarrow} \mathscr{W} \longrightarrow 0 .
$$

As it is precisely the complex of sheaves of $\mathbb{C}$-modules underlying the $G$-sheaf complex (A.2), the complex (A.2) must also be exact.

Proposition A.2 naturally generalises to flat families of finite sheaves. Let $S$ be a scheme of finite type over $\mathbb{C}$ endowed with a trivial $G$-action. Let $\mathscr{F}$ be a coherent $G$-sheaf on $S \times \mathbb{C}^{n}$, flat over $S$, whose fiber $\mathscr{F}_{\mid p}$ over any closed point $p \in Y$ is a finite-length $G$ sheaf on $\mathbb{C}^{n}$. Imitating the construction above, let $\mathscr{L}$ be the locally-free sheaf $\pi_{S}^{*} \pi_{S *} \mathscr{F}$ and let $\mathscr{L} \stackrel{\alpha}{\rightarrow} \mathscr{F}$ be the surjection map defined by adjunction.

There are two natural actions of $R$ on $\mathscr{L}$. The first action is via the inclusion $R \hookrightarrow \Gamma\left(\mathcal{O}_{S \times \mathbb{C}^{n}}\right)$ induced by the pullback from $\mathbb{C}^{n}$. The second action is via the inclusion $R \hookrightarrow \pi_{S *} \mathcal{O}_{S \times \mathbb{C}^{n}}$ which acts on $\pi_{*} \mathscr{F}$ and subsequently on $\mathscr{L}=\pi_{S}^{*} \pi_{S *} \mathscr{F}$. For any $r \in R$ and $s \in \mathscr{L}$ we denote the first action by $r \cdot s$ and the second action by $r \cdot 2 s$.

Corollary A.3. $\quad \mathscr{F}$ has a G-equivariant locally-free resolution given by

$$
0 \longrightarrow \wedge^{n} V_{\text {giv }} \otimes \mathscr{L} \stackrel{\delta_{n}}{\longrightarrow} \cdots \stackrel{\delta_{k+1}}{\longrightarrow} \wedge^{k} V_{\text {giv }} \otimes \mathscr{L} \stackrel{\delta_{k}}{\longrightarrow} \cdots \stackrel{\delta_{1}}{\longrightarrow} \mathscr{L} \stackrel{\alpha}{\longrightarrow} \mathscr{F} \longrightarrow 0
$$

where the maps $\delta_{k}$ are defined for $m_{i} \in V_{\text {giv }}^{\vee}$ and $s \in \mathscr{L}$ by

$$
\left(m_{1} \wedge \cdots \wedge m_{k}\right) \otimes s \mapsto \sum_{i=1}^{k}(-1)^{i+1}\left(\ldots m_{i-1} \wedge m_{i+1} \ldots\right) \otimes\left(m_{i} \cdot 2 s-m_{i} \cdot s\right)
$$

A.2. Proof of Proposition 2.1. As an application of the results in previous section, we use them to give an alternative proof of Proposition 2.1.

Proof of Proposition 2.1. By Corollary A.3 we have a locally free resolution of $\mathscr{F}$. Taking its dual we find that $\mathscr{F}^{\vee}[n]$ is isomorphic in $D^{G}\left(Y \times \mathbb{C}^{n}\right)$ to

$$
0 \longrightarrow \mathscr{L}^{\vee} \stackrel{\delta_{1}^{\vee}}{\longrightarrow} \cdots \stackrel{\delta_{k}^{\vee}}{\longrightarrow} \wedge^{k} V_{\text {giv }}^{\vee} \otimes \mathscr{L}^{\vee} \stackrel{\delta_{k+1}^{\vee}}{\longrightarrow} \cdots \stackrel{\delta_{n}^{\vee}}{\longrightarrow} \wedge^{n} V_{\text {giv }}^{\vee} \otimes \mathscr{L}^{\vee} \longrightarrow 0
$$

where the rightmost term, $\wedge^{n} V_{\text {giv }}^{\vee} \otimes \mathscr{L}^{\vee}$, lies in degree zero.

On the other hand, by definition $\pi_{Y *} \tilde{\mathscr{F}}=\left(\pi_{Y_{*}} \mathscr{F}\right)^{\vee}$, which is locally free. Hence $\pi_{Y}^{*} \pi_{Y *} \tilde{\mathscr{F}}=\mathscr{L}^{\vee}$. Applying Corollary A.3 again we find that $\tilde{\mathscr{F}}$ has a locally free resolution

$$
0 \longrightarrow \wedge^{n} V_{\text {giv }} \otimes \mathscr{L}^{\vee} \stackrel{\gamma_{n}}{\longrightarrow} \cdots \stackrel{\gamma_{k+1}}{\longrightarrow} \wedge^{k} V_{\text {giv }} \otimes \mathscr{L}^{\vee} \stackrel{\gamma_{k}}{\longrightarrow} \cdots \stackrel{\gamma_{1}}{\longrightarrow} \mathscr{L}^{\vee} \longrightarrow 0
$$


where differential maps $\gamma_{i}$ are defined by (A.5), with the action $\cdot 2$ of $R$ on $\mathscr{L}^{\vee}$ being induced by the action (2.3) of $R$ on $\operatorname{Hom}_{\mathcal{O}_{Y}}\left(\pi_{*} \mathscr{F}, \mathcal{O}_{Y}\right)$.

We now prove that these two complexes are isomorphic, thus showing $\mathscr{F}^{\vee}[n] \simeq \tilde{\mathscr{F}}$. Let $\phi$ be the interior product isomorphism $\wedge^{k} V_{\text {giv }}^{\vee} \otimes \wedge^{n} V_{\text {giv }} \rightarrow \wedge^{n-k} V_{\text {giv }}$. It is $G$-equivariant. Since $G \subseteq \mathrm{SL}_{n}(\mathbb{C}), \wedge^{n} V_{\text {giv }}$ is trivial as a representation of $G$. Thus we obtain $G$-equivariant isomorphisms $\wedge^{k} V_{\text {giv }}^{\vee} \stackrel{\sim}{\rightarrow} \wedge^{n-k} V_{\text {giv }}$ which induce isomorphisms $\alpha_{i}: \wedge^{n+i} V_{\text {giv }}^{\vee} \otimes \mathscr{L}^{\vee} \stackrel{\sim}{\rightarrow} \wedge^{-i} V_{\text {giv }} \otimes \mathscr{L}^{\vee}$

It remains only to verify that $\alpha_{i}$ defines a chain map between the two complexes, i.e. that the following diagram commutes:

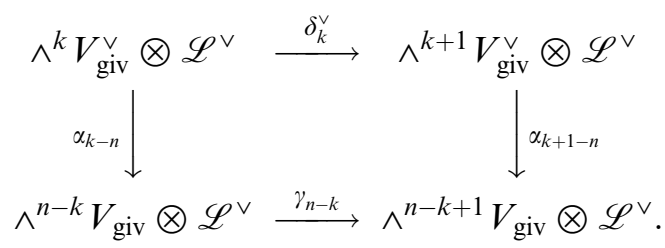

It is a straightforward calculation which we leave to the reader.

\section{References}

[1] T. Bridgeland, A. King, and M. Reid, The McKay correspondence as an equivalence of derived categories, J. Amer. Math. Soc. 14 (2001), 535-554.

[2] A. Craw and A. Ishii, Flops of $G$-Hilb and equivalences of derived category by variation of GIT quotient, Duke Math J. 124 (2004), no. 2, 259-307.

[3] A. Craw, D. Maclagan, and R. R. Thomas, Moduli of McKay quiver representations I: the coherent component, Proc. London Math. Soc. 95 (2007), no. 1, 179-198.

[4] A. Craw, An explicit construction of the McKay correspondence for $A$-Hilb $\mathbb{C}^{3}$, J. Algebra 285 (2005), no. 2, 682-705.

[5] P. Deligne, Cohomologie a support propre et construction du foncteur $f^{!}$, in: Residues and Duality, R. Hartshorne, Springer (1966), 404-421.

[6] G. Gonzales-Sprinberg and J.-L. Verdier, Construction géométrique de la correspondance de McKay, Ann. Sci. ENS 16 (1983), 409-449.

[7] $Y$. Ito and M. Reid, The McKay correspondence for the finite subgroups of $\operatorname{SL}(3, \mathbb{C})$, Higher-dimensional complex varieties (Trento 1994), de Gruyter (1996), 221-240.

[8] T. Logvinenko, Families of $G$-constellations over resolutions of quotient singularities, preprint 2003.

[9] T. Logvinenko, Derived McKay correspondence via pure-sheaf transforms, Math. Ann., to appear.

[10] T. Logvinenko, Natural $G$-constellation families, preprint 2006.

[11] J. McKay, Graphs, singularities and finite groups, Proc. Symp. Pure Math. 37 (1980), 183-186.

[12] M. Reid, Young person's guide to canonical singularities, Proc. Symp. Pure Math. 46 (1987), $345-414$.

[13] M. Reid, McKay correspondence, preprint 1997.

[14] M. Sweedler, Hopf algebras, W.A. Benjamin Inc., 1969.

Department of Mathematics, Rice University, Houston, TX e-mail: scautis@math.harvard.edu

Department of Mathematics, KTH, Stockholm, Sweden e-mail: tlog@kth.se 